\title{
Comparison of APD Macromodels for Accuracy, Speed and Implementation Issues
}

\author{
by \\ Dragan Trifkovic
}

A thesis submitted to the Faculty of Graduate Studies and Research in partial fulfillment of the requirements for the degree of Master of Applied Sciences

Ottawa-Carleton Institute for Electrical and Computer Engineering Department of Electronics

Faculty of Engineering

Carleton University

Ottawa, Ontario, Canada

June 2012

(C) Dragan Trifkovic 2012 
Library and Archives

Canada

Published Heritage

Branch

395 Wellington Street

Ottawa ON K1A ON4

Canada
Bibliothèque et

Archives Canada

Direction du

Patrimoine de l'édition

395 , rue Wellington

Ottawa ON K1A ON4

Canada
Your file Votre référence

ISBN: 978-0-494-93505-7

Our file Notre référence

ISBN: 978-0-494-93505-7
NOTICE:

The author has granted a nonexclusive license allowing Library and Archives Canada to reproduce, publish, archive, preserve, conserve, communicate to the public by telecommunication or on the Internet, loan, distrbute and sell theses worldwide, for commercial or noncommercial purposes, in microform, paper, electronic and/or any other formats.

The author retains copyright ownership and moral rights in this thesis. Neither the thesis nor substantial extracts from it may be printed or otherwise reproduced without the author's permission.
AVIS:

L'auteur a accordé une licence non exclusive permettant à la Bibliothèque et Archives Canada de reproduire, publier, archiver, sauvegarder, conserver, transmettre au public par télécommunication ou par l'Internet, prêter, distribuer et vendre des thèses partout dans le monde, à des fins commerciales ou autres, sur support microforme, papier, électronique et/ou autres formats.

L'auteur conserve la propriété du droit d'auteur et des droits moraux qui protege cette thèse. $\mathrm{Ni}$ la thèse ni des extraits substantiels de celle-ci ne doivent être imprimés ou autrement reproduits sans son autorisation.
In compliance with the Canadian Privacy Act some supporting forms may have been removed from this thesis.

While these forms may be included in the document page count, their removal does not represent any loss of content from the thesis.
Conformément à la loi canadienne sur la protection de la vie privée, quelques formulaires secondaires ont été enlevés de cette thèse.

Bien que ces formulaires aient inclus dans la pagination, il n'y aura aucun contenu manquant. 


\begin{abstract}
With the high data rates optical communication requires, there is a greater need for sophisticated Avalanche Photodiodes (APDs). The effects such as the electron and hole transit times, the avalanche buildup time, the hole trapping and the capacitive resistive and inductive parasitics are the limiting factors of APD speed. If these effects are not considered in the design, the consequences may lead to Inter Symbol Interference on the output. Therefore a time domain simulation must be performed on the APD to predict ISI.
\end{abstract}

In order to perform a time domain simulation of APD, an accurate, fast APD model is needed for the simulation of mixed optical-electrical circuits. The traditional methods such as Fast Fourier Transform APD (FFT-APD) and Circuit Equivalent APD (CE-APD) macromodels have been used for this application. Experience has shown that these methods have drawbacks.

In this thesis, two more recent APD macromodel are reviewed. The first recent macromodel was previously developed based on a modification of CE-APD. The second APD macromodel was developed using recursive convolution( $\mathrm{RC})$. The four $\mathrm{APD}$ macromodels have been compared for accuracy and simulation speed and implementation issues. The first task focused on APD reliability by comparing the accuracy and simulation speed for variation of signal input, nonlinear load and APD parameters. The second task focused on comparing the accuracy and simulation speed between 
the macromodels with variable time step and fixed time step. 
Dedicated to my Family 


\section{Acknowledgments}

I would like to thank my supervisors, Professor Tom Smy and Professor Pavan Gunupudi for their guidance and motivation throughout this research.

I wish to thank my colleagues A. Narayanan, M. Adel and A. Saini for meticulously proof reading this thesis. I would like to acknowledge and thank my colleagues A. Sridhar, H. Dhindsa, B. Nouri, Y. Hong, L. Filipovic, A. Charest, J. J. Kang, M. Siddique, V. Gongal and D. Paul for the helpful discussions and relaxing chats which made my research at Carleton a pleasant and enjoyable experience.

I wish to thank my father, Jefto and my sisters Jelena and Dragana for their patience, affection and guidance. I would also like to thank all of my friends I. Milasin, L. Krzesinski, A. Hill, M. Lepine, S. Lepine, P. Anweiler and the rest of the crew for helping balance my research work and my social life. 


\section{Contents}

1 Introduction $\quad 1$

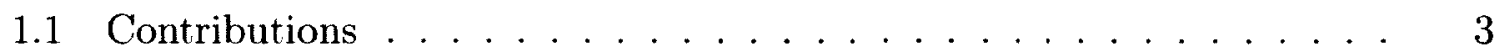

1.2 Organization of the Thesis . . . . . . . . . . . . . . 4

2 Review of Avalanche Photodiode Simulation $\quad 5$

2.1 Review of APD Physics ........................ 6

2.2 Time Domain APD Model . . . . . . . . . . . . . . . . . . 10

2.3 Review of SAM APD Time Domain Model . . . . . . . . . . . . 10

2.3.1 Review of SAGCM APD Time Domain Model . . . . . . . . 14

2.4 Frequency APD Model . . . . . . . . . . . . . . . 17

2.4 .1 SAM APD ...................... 17

2.4 .2 SAGCM-APD .................... 18

2.4.3 APD Model Stability . . . . . . . . . . . . . . 20

2.5 Review of Time Domain APD Modelling Techniques . . . . . . . . . 21 
2.5.1 Review of Traditional Convolution and the FFT-APD Model . 22

2.5.2 Review of Circuit Equivalent APD . . . . . . . . . . 23

3 QC-APD Macromodel

3.1 The Origin of QC-APD Macromodel ............. . . 31

3.1.1 Time Domain Simulation of SAGCM-APD using CE-APD Macro-

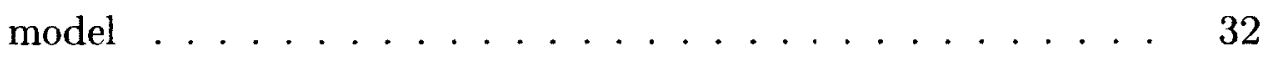

3.1 .2 Fixing CE-APD Macromodel .............. 36

3.2 Effects of $N(t)$ Segmentation on Macromodel Performance . . . . . . 43

3.2.1 Simulation Processing Time and Accuracy Results for Different $N(t)$ Segments . . . . . . . . . . . . . .

4 Recursive Convolution APD Macromodel $\quad 48$

4.1 Review of RC-APD macromodel . . . . . . . . . . . . . 49

4.2 Review of RC Technique . . . . . . . . . . . . . . . 49

4.3 Review of InP, InGaAsP and InGaAs APD . . . . . . . . . 51

4.4 Review of Vector Fit . . . . . . . . . . . . . . 52

4.5 Development of RC based APD Time Domain Model . . . . . . . . 55

4.6 Testing Accuracy and Simulation Processing Time of RC-APD Macromodel ...................... 57

4.6.1 Simulation Results of Accuracy Control . . . . . . . . . . . . 59 
4.6.2 Simulation Results of Simulation Processing Time . . . . . 61

4.7 Summary of Results . . . . . . . . . . . . . . . 62

5 Macromodel Comparison for Simulation Processing Time, Accuracy and Macromodel Implementation Issues

5.1 Methods of Comparison ................. 64

5.1.1 Fixed Time Stepping ................. 65

5.1.2 Variable Time Stepping . . . . . . . . . . . 66

5.2 Time Domain Simulation of APD with a Linear Load . . . . . . . . 67

5.2.1 Simulation Processing Time Results . . . . . . . . . . . 70

5.2.2 Accuracy Results of the Three Macromodels ......... 71

5.3 Circuit Simulation of an APD terminated with Shunt Feedback TIA . 78

5.3.1 Simulation Processing Time Results . . . . . . . . . . . . 80

5.3.2 Accuracy of Models ................... 82

5.4 Macromodel Reliability and Implementation Issues . . . . . . . . . . 89

5.4.1 Macromodel Reliability Check with Fast Input Pulses . . . . . 91

5.4.2 Reliability Check for Slow Input . . . . . . . . . . . . . 94

5.5 Effects of Simulation Step Size Control on APD Models . . . . . . . 96

5.5.1 Uniform Step Size Simulation . . . . . . . . . . . . 96

5.5.2 Simulation Example with Non Uniform Simulation Step Size . $\quad 97$ 
6 Conclusion and Future Work 102

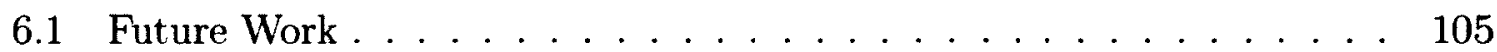

$\begin{array}{ll}\text { A Review of TIA } & 106\end{array}$

$\begin{array}{ll}\text { References } & 108\end{array}$ 


\section{List of Figures}

2.1 (a) SAM-APD structure. (b) The plot of space charge density across the depletion region. (c) The plot of electric field across the depletion

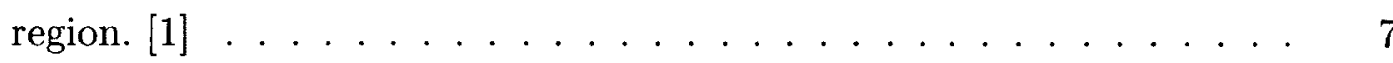

2.2 (top) Generation of primary EHP. (bottom) Generation of secondary EHPs from primary EHP. $[1][2] \ldots \ldots \ldots \ldots \ldots$

2.3 APD modelling characterization of physical parameters [2] . . . 11

2.4 Resistive, capacitive and inductive parasitics in a circuit [2] . . . 14

2.5 Basic structure of SAGCM APD from $[3] \ldots \ldots \ldots$

2.6 Basic structure of SAGCM-APD [3] $\ldots \ldots \ldots \ldots \ldots$

2.7 Basic block diagram of SAGCM APD $[3] \ldots \ldots \ldots \ldots$

2.8 Synthesis of the frequency equivalent delay block $\ldots \ldots \ldots 25$

2.9 Synthesis of the frequency equivalent integrator block . . . . . . 26

2.10 Synthesis of the frequency stable RL reasonator block . . . . . . 27

2.11 Synthesis of the frequency equivalent unstable RL resonator block . . $\quad 27$ 
2.12 Synthesis of the constant gain block . . . . . . . . . . . . . 28

2.13 Synthesis of the frequency equivalent differentiator block . . . . . . 29

3.1 Circuit topology of SAGCM-APD that is connected to a $1 \Omega$ resistor . 32

3.2 Time domain response of a trapezoidal pulse with a simple resistive load using the FFT-APD and CE-APD models . . . . . . . . . 34

3.3 Block diagram of the SAM-APD [2] . . . . . . . . . . 35

3.4 Discretizing of $N(t)$ function into rectangular pulses . . . . . . . . 37

3.5 The time domain convolution between $P_{\text {in }}(t)$ and $N_{\text {discrete }}(t) \ldots . . . \quad 38$

3.6 The time domain convolution between $P_{\text {in }}(t)$ and one discretized $N_{\text {segl }}(t)$ segment with amplitude A . . . . . . . . . . . . 38

3.7 Circuit synthesized from term 1 in $(3.5) \ldots \ldots . \ldots 40$

3.8 Circuit synthesized from term 2 in $(3.5) \ldots \ldots . . . . . . . .40$

3.9 Sub-circuit of $N_{\text {seg1 }}(t)$ with $P_{\text {in }}(t)$ input . . . . . . . . . . . 41

3.10 Circuit implementation of $N_{\text {discrete }}(t)$ with $P_{i n}(t)$ input . . . . . . 42

3.11 Circuit topology of APD with linear load . . . . . . . . . . . . 44

3.12 The APD step response with different number of segments $N$ seg . . 46

3.13 The simulation processing time of APD step response with different number of segments $N_{\text {seg }} \ldots \ldots \ldots$. . . . . . . . . 47

4.1 Development of RC-APD macromodel . . . . . . . . . . . 56 
4.2 Circuit topology of RC-APD with resistive load . . . . . . . . . 57

4.3 Effects of number of pole changes on accuracy of time domain response using RC-APD macromodel as compared to FFT-APD . . . . . . . . 59

4.4 Effects of number of pole changes on accuracy of time domain response using RC based APD macromodel as compared to FFT-APD . . . . 60

4.5 Number of fitted poles vs simulation processing time of RC based APD macromodel ..................... 62

5.1 Circuit of APD terminated with a linear load . . . . . . . . 68

5.2 Simulation processing time (sec) versus the number of simulation time points for FFT, QC-APD and RC based APD . . . . . . . . . 70

5.3 Time Domain Response of Circuit from Figure 5.1 . . . . . . . . 72

5.4 RC-APD response for different number of fitted poles . . . . . . . 73

5.5 RC-APD response for different number of fitted poles . . . . . . . 74

5.6 QC-APD response for different number of segments . . . . . . 75

5.7 QC-APD response for different number of segments . . . . . . . 76

5.8 QC-APD response for different number of segments . . . . . . . 77

5.9 Circuit topology of APD with shunt feedback TIA . . . . . . . 79

5.10 simulation processing time of All the three APD models(with TIA)

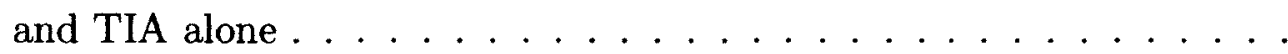


5.11 The response of the APD FFT-APD, QC-APD and RC-APD model with TIA load $\ldots \ldots \ldots \ldots \ldots \ldots \ldots \ldots \ldots \ldots \ldots$

5.12 RC-APD response for 2 and 4 fitted poles is compared with FFT-APD response $\ldots \ldots \ldots \ldots \ldots \ldots \ldots \ldots \ldots$

5.13 RC-APD response for 8, 16 and 20 fitted poles is compared with FFTAPD response $\ldots \ldots \ldots \ldots \ldots \ldots \ldots \ldots$

5.14 QC-APD response for 1 and 2 segments is compared with FFT-APD response $\ldots \ldots \ldots \ldots \ldots \ldots \ldots \ldots$

5.15 QC-APD response for 3 and 4 segments is compared with FFT-APD response $\ldots \ldots \ldots \ldots \ldots \ldots \ldots \ldots \ldots$

5.16 QC-APD response for 5,10 and 20 segments is compared with FFTAPD response $\ldots \ldots \ldots \ldots \ldots \ldots \ldots \ldots$

5.17 Circuit topology APD and TIA for macromodel reliability testing . .

5.18 The time domain responses of FFT-APD, QC-APD and RC-APD

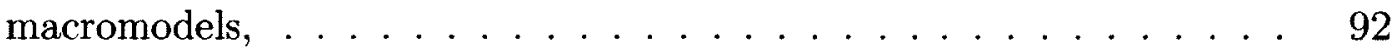

5.19 Time domain response of FFT-APD,RC-APD and QC-APD . . . . 93

5.20 Time domain response of FFT-APD, RC-APD and QC-APD $\ldots . .95$

5.21 Time domain responses of RC-APD with different uniform simulation

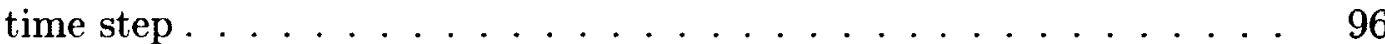


5.22 Time domain APD response of QC-APD with different uniform simulation time step $\ldots \ldots \ldots \ldots \ldots \ldots \ldots$

5.23 Time domain APD response of QC-APD with non uniform time step for a 1 psec rise time input $\ldots \ldots \ldots \ldots \ldots \ldots$

5.24 Time domain APD current response of QC-APD with non uniform time step for a $1 p s e c$ rise time input $\ldots \ldots \ldots \ldots \ldots$

A.1 General shunt feedback TIA (a) and shunt feedback with common source amplifier $(b) \ldots \ldots \ldots \ldots \ldots \ldots \ldots$ 


\section{List of Tables}

3.1 Physical parameters of the SAGCM-APD [3] $\ldots \ldots \ldots . \ldots 33$

3.2 Physical parameters of the InP/InGaAsP/InGaAs APD [2] . . . . 44

3.3 Computer hardware used in simulation processing time measurements

4.1 Computer hardware used in simulation processing time measurements

4.2 Physical parameters of the InP/InGaAsP/InGaAs APD . . . . . 58

4.3 Example 1:RC based APD model parameters . . . . . . . . . . 58

5.1 Physical parameters of the InP/InGaAsP/InGaAs APD [2] . . . . 68

5.2 QC-APD macromodel parameters . . . . . . . . . . . . 69

$5.3 \quad$ FFT-APD macromodel parameters . . . . . . . . . . . . . 69

5.4 RC-APD macromodel parameters . . . . . . . . . . . . 69

5.5 Computer hardware used in simulation processing time measurements 69

5.6 Macromodel speedup comparison . . . . . . . . . . . 71

5.7 Computer hardware used in simulation processing time measurements $\quad 79$ 
5.8 APD macromodel speedup comparison . . . . . . . . . . . . . 81

5.9 Comparing simulation processing time contribution of each APD macro-

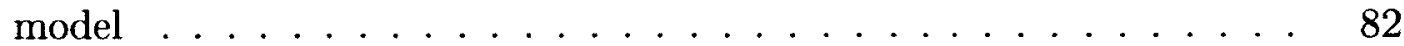

5.10 Constant physical parameters for implementation issues testing . . 90

5.11 Example 1:QC-APD model parameters . . . . . . . . . . . 90

5.12 FFT-APD model parameters . . . . . . . . . . . . . 90

5.13 RC-APD model parameters . . . . . . . . . . . . . 90

5.14 Table describing the fast and the slow pulse . . . . . . . . . . . 91

5.15 Variable simulation parameter for testing macromodel reliability . . . 91

5.16 Comparing APD models . . . . . . . . . . . . . . 100 


\title{
List of Symbols
}

\author{
$\eta \quad$ External Quantum Efficiency \\ $P_{\text {in }} \quad$ Incident optical pulse energy $(\mathrm{J})$ \\ $h v \quad$ Photon energy (J) \\ $\alpha \quad$ Absorption Coefficient $(1 / \mathrm{m})$ \\ $\omega \quad$ Angular frequency ( $\mathrm{rad} / \mathrm{sec})$ \\ $q \quad$ Electronic charge (C) \\ h Hole carrier representation \\ e Electron carrier representation \\ $M_{0} \quad$ DC gain of the APD \\ $p(x) \quad$ Net charge distribution function across depletion region (C) \\ E $\quad$ Electric field inside APD $(\mathrm{V} / \mathrm{m})$ \\ $u(t) \quad$ Unit Step function (s) \\ $v_{p} \quad$ Hole saturated drift velocity $(\mathrm{m} / \mathrm{s})$ \\ $v_{n} \quad$ electron saturated drift velocity $(\mathrm{m} / \mathrm{s})$
}


xvii

$\begin{array}{ll}R & \text { Resistive parasitic of the APD ( } \Omega \\ L & \text { Inductive parasitic of the APD (H) } \\ C & \text { Capacitive parasites of the APD (F) } \\ W, x_{a} & \text { Width of the Depletion Region (m) } \\ W_{a} & \text { Width of the Absorption Region (m) } \\ x_{m} & \text { Width of the Multiplication Region (m) } \\ e_{h} & \text { Effective emission rate of hole trapping }(1 / \mathrm{s}) \\ t_{m} & \text { Avalanche buildup time taken when } M_{0}=2(\mathrm{~s}) \\ i_{p h} & \text { Photogenerated current (A) } \\ x_{t} & \text { Location of the interface between the Transition region and Absorption Re- } \\ & \text { Fion (m) } \\ \mathcal{F}\{.\} & \text { Angular frequency of the phasor (rad/sec) } \\ \omega & \text { Laplace variable } s=j \omega .\end{array}$




\section{Abbreviations}

$\begin{array}{ll}\text { APD } & \text { Avalanche Photodiode } \\ \text { ISI } & \text { Inter Symbol Interference } \\ \text { SAGCM } & \text { Separate Absorption Graded Charge Multiplication } \\ \text { EHP } & \text { Electron Hole Pair } \\ \text { RC } & \text { Recursive Convolution } \\ \text { FFT } & \text { Fast Fourier Transform } \\ \text { IFFT } & \text { Inverse Fast Fourier Transform } \\ \text { MNA } & \text { Modified Nodal Analysis } \\ \text { VF } & \text { Vector Fit } \\ \text { PU } & \text { Pulse } \\ \text { CE-APD } & \text { Circuit Equivalent Avalanche Photodiode } \\ \text { QC-APD } & \text { Quasi Circuit Avalanche Photodiode } \\ \text { RC-APD } & \text { Recursive Convolution Avalanche Photodiode } \\ \text { FFT-APD } & \text { Fast Fourier Transform Avalanche Photodiode }\end{array}$


TIA Transimpedance Amplifier

NMOS Donnor( $N$ type) Metal Oxide Silicon

MOSFET Metal Oxide Silicon Field Effect Transistor

SPICE Simulation Program on Integrated Circuit Emphesis

HSPICE Circuit Simulator developed by Synopsys

OptiSPICE Optoelectronic Circuit Simulator developed by Optiwave 


\section{Chapter 1}

\section{Introduction}

Over the years, there has been a great interest in the development of avalanche photodiodes (APD) for high data optical communication systems.

The APD's internal gain provides greater sensitivity than the PIN photodiode and thus it is used in optical receiver design. The drawback of APD is the fact it slows down the receiver response. The APD speed is limited by effects such as the electron and hole transit times, the avalanche buildup time, the hole trapping and the capacitive and inductive parasitics. The high speed receiver requirements have led to the development of more sophisticated APD devices which improve upon the APD speed limitations.

If the APD limiting effects are not properly considered in the receiver design, the consequence may lead to Inter Symbol Interference (ISI). ISI is easily detectable in 
time domain. Therefore a time domain simulation can be performed to predict ISI.

Detailed transient simulation of electrical circuits is typically done using simulation tools developed from SPICE [4]. These SPICE-like tools link electrical devices represented by physical models into a set of non-linear ordinary differential equations which are first order in time. The set of equations are then integrated in time with Newton-Raphson iterations used at each time point to accommodate the nonlinearities.

A key factor in the effective use of SPICE-like simulators is small, efficient and accurate macro-models of the active devices. An accurate, fast and computationally robust photodiode model is essential to the simulation of mixed optical-electrical circuits. In this work, two simulation programs will be used. HSPICE, a commercial program [5] [6], will be used for purely electrical circuits and primarily as a baseline simulator. The second simulator is OptiSPICE [7], a simulator which has extended the SPICE formulation to mixed optical-electrical circuits. OptiSPICE has a large set of optical and electrical models and is well suited for testing and comparing APD models.

This thesis will be concerned with the development and comparison of four differently formulated photodiode models. The first conventional macromodel for the time domain simulation was developed using Fast Fourier Transform (FFT) [2]. The second conventional APD macromodel was developed for a Separate Absorption Grad- 
ing Charge Multiplication Avalanche Photodiode (SAGCM) APD by synthesizing the APD frequency into an equivalent circuit (CE-APD). Both of these models will be shown to have significant drawbacks.

As an alternative to these two models this thesis will present and evaluate two APD macromodels: 1) a previously developed model based on a synthesized circuit (quasicircuit or QC-APD), but formulated in a manner which produces stable operation; and 2) a new Recursive Convolution (RC) APD model. A comparison of macromodels (FFT, Circuit, Quasi-Circuit and RC) is performed based on characteristics such as speed, accuracy and implementation issues. The first task is to compare the accuracy of QC-APD and RC-APD with varying input signal pulses, nonlinear loads and APD device parameters. The second task is to compare the simulation speed and accuracy of QC-APD and RC-APD with variable and fixed time-stepping.

\subsection{Contributions}

In this thesis, comparison of APD macromodels based on FFT, explicit circuit implementation, QC-APD and Recursive Convolution are compared for simulation speed, accuracy and implementation issues. The specific contributions made in this thesis are listed below.

1. Development of RC-APD model 
2. Comparison of FFT-APD, CE-APD, QC-APD and RC-APD models for simulation processing time and speedup.

3. Comparison of FFT-APD, CE-APD, QC-APD and RC-APD models for accuracy

4. Review of QC-APD and RC-APD for implementation issues.

\subsection{Organization of the Thesis}

This thesis is organized as follows: Chapter 2 provides a review of APD physics, of time and frequency domain physical APD models and a review of the macromodels (FFT-APD and CE-APD) that were presented in [2] and [3]. Chapter 3 provides a review of development of QC-APD and presents the macromodel accuracy and simulation processing time. Chapter 4 presents the development of RC-APD macromodel and provides the macromodel accuracy and simulation processing time. Chapter 5 provides a comparison of CE-APD, QC-APD, FFT APD and RC-APD for simulation processing time, accuracy and implementation issues. Chapter 6 presents a conclusion of the results and describes possible future research work. 


\section{Chapter 2}

\section{Review of Avalanche Photodiode}

\section{Simulation}

APD performance is important in the design of optical communication systems because it effects the sensitivity and speed of the system response. In order to analyze the system response, the overall system must be simulated.

Generally, a system is composed of many linear and nonlinear blocks. The system nonlinearity can cause inter symbol interference (ISI). In order to predict ISI, the system needs to be simulated with full nonlinear effects. This necessitates the need to perform transient analysis for the entire system.

In order to perform system transient analysis, the APD model must have a fast, accurate and robust macromodel for circuit simulation. For effective simulation an 
APD device model has to consider physical effects such as avalanche build up time, hole trapping, transit time effects, and resistive, inductive and capacitive parasitics. In this chapter, the first section will review the APD physics and the time and frequency domain APD models. The second section will review the development of an FFT-APD and CE-APD macromodel.

\subsection{Review of APD Physics}

APD behaviour is strongly governed by its physical structure, material composition and its voltage bias. A simplified APD structure is composed of at least 4 different layers, metal contacts on each terminal and an optical connection (gap between the metal contacts and $p^{+}$layer), as shown in Figure 2.1 (a). 


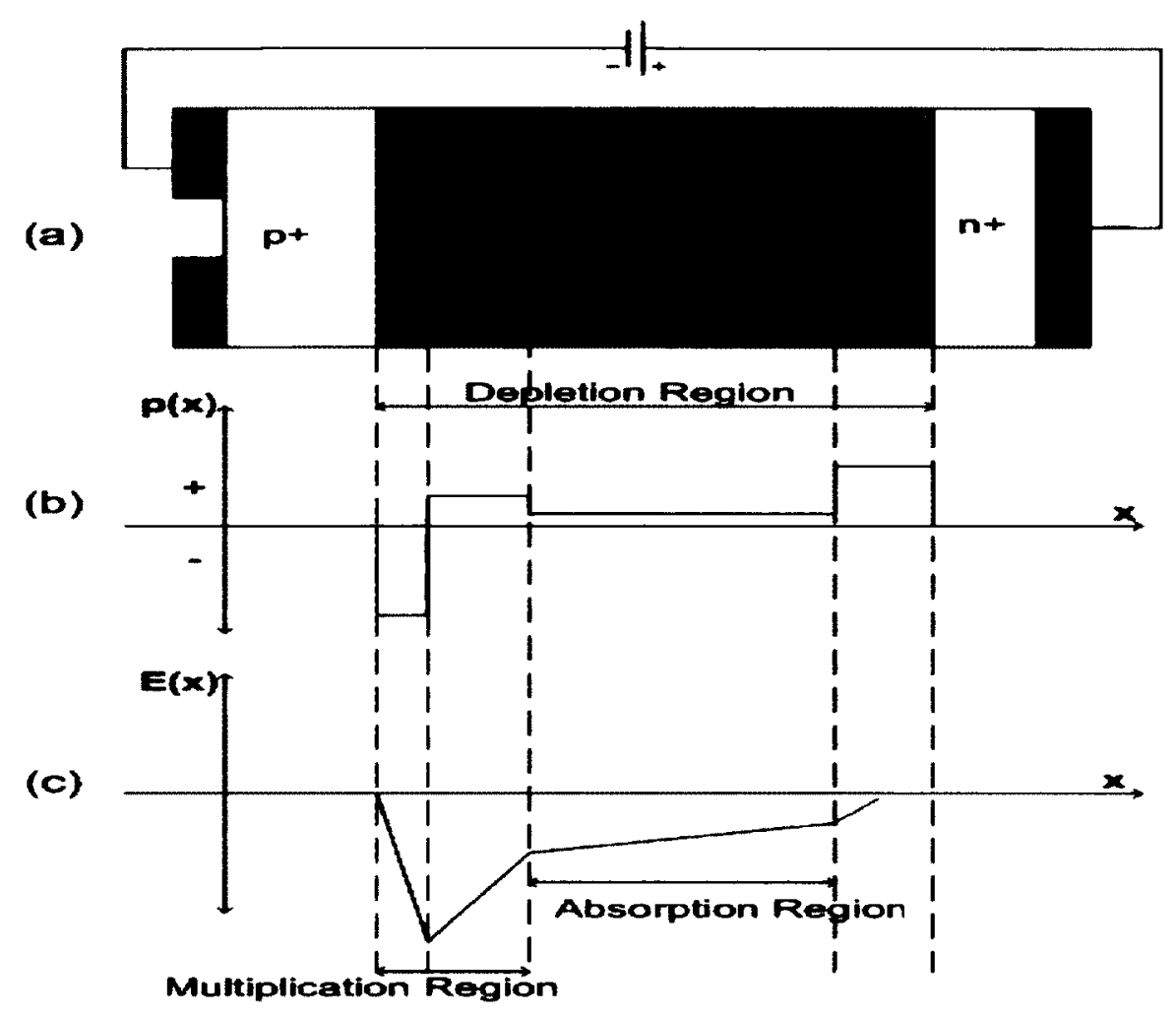

Figure 2.1: (a) SAM-APD structure. (b) The plot of space charge density across the depletion region. (c) The plot of electric field across the depletion region. [1]

The first layer is a thin heavily doped $p^{+}$type region, the second layer is a thin medium doped $\mathrm{n}$ type region, the third region is a lightly doped $\mathrm{n}$ type region that is almost intrinsic region and the fourth region is heavily doped large $n^{+}$region. The APD structure, as shown in Figure 2.1 (a), must be reversed bias such that the depletion region is generated from $p^{+}$to the $n^{+}$layer. The net space charge distribution function, $p(x)(\mathrm{C})$, within the depletion region is shown in Figure 2.1 (b), where the function is assumed to be constant for each region. 


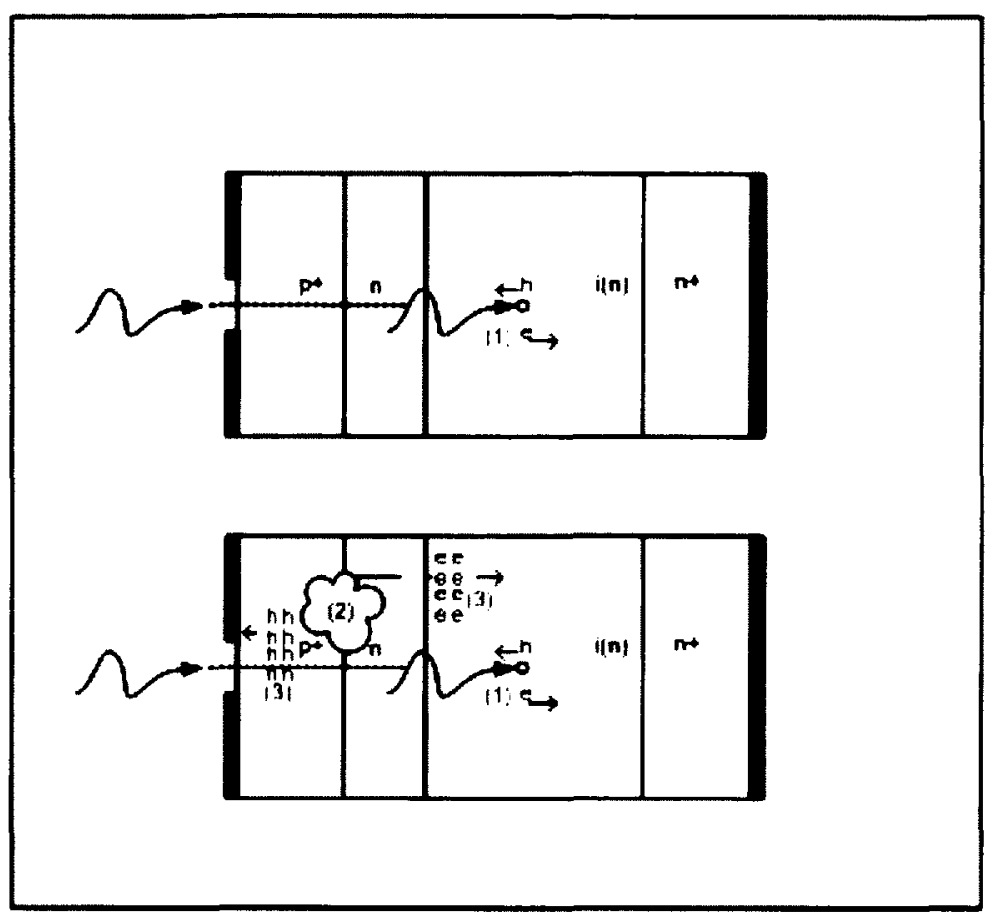

Figure 2.2: (top) Generation of primary EHP. (bottom) Generation of secondary EHPs from primary EHP. [1] [2]

The integration of the net space charge distribution $p(x)$ is equal to the electric field function. $E(x)$ where $\mathrm{x}$ is the distance across the depletion region. The regions with the strong electric field presence, as shown in Figure 2.1 (c) are the multiplication and absorption regions. The APD without the optical signal behaves just like a regular reverse biased diode, where a very small dark current is generated. Therefore the source of the APD current will be initiated by photons.

The photon can only be absorbed in the material with a lower bandgap energy. The material with greater bandgap is transparent and transmits the light. In [2], the $p^{+}, \mathrm{n}, \mathrm{i}-\mathrm{n}$ and $n^{+}$is composed of the InP, InGaAsP, InGaAs and InGaAs and the 
wavelength range of application is between 1.3 and $1.55 \mu \mathrm{m}$. The InP and InGaAsP layers will transmit the photon because their bandgap energies are larger than the photon energy. When the photon reaches the InGaAs, i-n, material with a smaller bandgap, the photon can be absorbed and create an electron hole pair (EHP) as shown in Figure 2.2 (top).

The strong electric field within (i-n) region will separate the electron and the hole and move them toward the neutral regions $n^{+}$and $p^{+}$as shown in Figure 2.2 . When the hole reaches the $\mathrm{n}$ region, the electric field becomes stronger and the hole receives enough kinetic energy to initiate an impact ionization on a InGaAsP covalent bond and generate another EHP. The new EHP will also get separated by the strong electric field and each carrier will move to their respective neutral region. The new EHP may create another EHP and this leads to an avalanche of impact ionizations as shown in Figure 2.2 (bottom), where a carrier, in this case a hole, created multiple EHPs as seen in Figure 2.2 (bottom).

The flow of carriers across the channel will generate the photocurrent that will be modelled in time and frequency domains. The next two sections will review the time and frequency domain models for two different APD structures. 


\subsection{Time Domain APD Model}

An APD model is developed for each unique APD device because the APD device's operation is strongly geometry dependant. In this section, the time domain models for a Separate Absorption Multiplication Avalanche Photodiode (SAM APD) structure and Separate Absorption Grading Charge Multiplication Avalanche Photodiode (SAGCM APD) structure will be reviewed.

\subsection{Review of SAM APD Time Domain Model}

The SAM APD structure is shown in Figure 2.3. Note that $x_{m}$ refers to the edge of the multiplication region, $x_{t}$ refers to the edge of the transition region and $x_{a}$ refers to the edge of absorption region. Thus the structure is composed of three major regions, the multiplication region (between 0 and $x_{m}$ ), the transition region (between $x_{m}$ and $x_{t}$ ) and the absorption region (between $x_{t}$ and $x_{a}$ ) as shown Figure 2.3. 


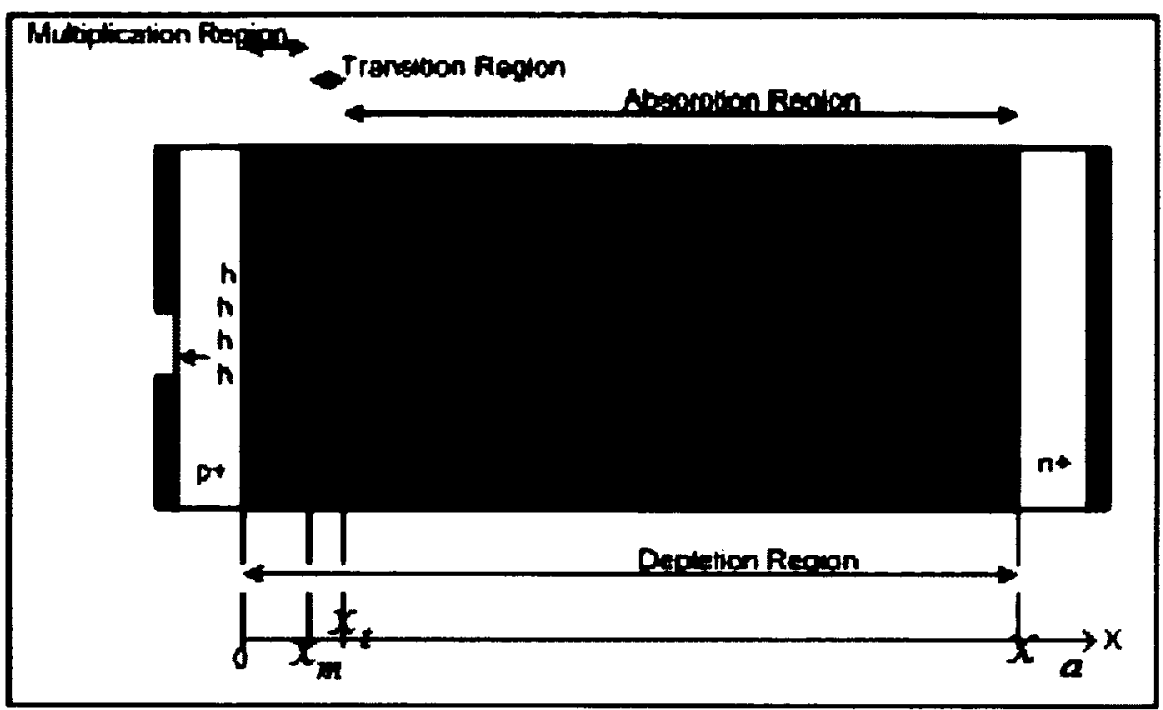

Figure 2.3: APD modelling characterization of physical parameters [2]

The time domain APD model is modelled at a specific bias point and has no explicit voltage dependance. The voltage bias across the APD is such that all the three regions are completely depleted up to a width $x=x_{a}$. In Figure 2.3, the incoming incident photon is absorbed completely inside the absorption region and generates an EHP. The absorption rate across the region is defined by the coefficient $\alpha$. The strong electric field in the absorption and multiplication region separate the electron and hole pairs towards each respective neutral regions as shown in Figure 2.3. When the hole reaches the transit region the impact ionization is initiated and eventually an avalanche of electrons generated by impact ionization reaches the multiplication region. This model stipulates that most of the secondary EHP from avalanche effect are created at or near the edge of multiplication region $(x=0)$ as shown in Figure 2.3. 
Thus the holes instantly move into the neutral region while the secondary electrons have to traverse the multiplication, transit and absorption region in order to reach the neutral region.

The photogenerated current, $i_{p h}(t)$, in [2] is determined by the superposition of all the carriers that traverse the depletion region as given in (2.1).

$$
i_{p h}(t)=\frac{q}{W}\left(v_{n} N(t)+v_{p} P(t)+v_{n} N_{s}(t)\right)
$$

where:

$i_{p h}(t)$ is the photogenerated current

$q$ is the electric charge

$v_{n}, v_{p}$ is the electron and hole carrier saturated drift velocity

$N(t), P(t)$ is the number of primary mobile electrons and holes

$N_{s}(t)$ is the number of secondary mobile electrons created from impact ionization

$W$ is the depletion region width $(\mathrm{m})$

The carrier mobility functions $N(t), N_{s}(t)$ and $P(t)$ given in [2] and [8] as shown in (2.2), (2.3) and (2.4).

$$
N(t)=\frac{\eta P_{i n}}{h v}\left[1-e^{-\alpha W_{a}+\alpha v_{n} t}\right]\left\{u(t)-u\left(t-\frac{W_{a}}{v_{n}}\right)\right\}
$$




$$
\begin{aligned}
& P(t)=\frac{\eta P_{i n}}{h v}\left[\left[\left[e^{-\alpha v_{p} t}-e^{-\alpha W_{a}}\right]+\left[1-e^{-e_{h} t}\right]\left[1-e^{-\alpha v_{p} t}\right]\right]\left\{u(t)-u\left(t-\frac{W_{a}}{v_{p}}\right)\right\}\right. \\
& +\left[1-e^{-a W_{a}}\right]\left[e^{-e_{h} \frac{W_{a}}{v_{p}}}-e^{-e_{h} t}\right]\left\{u\left(t-\frac{W_{a}}{v_{p}}\right)\right\} \\
& -\left[1-e^{-\alpha v_{p} t+\alpha x_{t}}\right]\left[1-e^{-e_{h} t+e_{h} \frac{x_{t}}{v_{p}}}\right]\left\{u\left(t-\frac{x_{t}}{v_{p}}\right)-u\left(t-\frac{W_{o}}{v_{p}}-\frac{x_{t}}{v_{p}}\right)\right\} \\
& \left.-\left[1-e^{-\alpha W_{a}}\right]\left[e^{-e_{h} \frac{W_{a}}{v_{p}}}-e^{-e_{h} t+e_{h} \frac{x_{t}}{v_{p}}}\right]\left\{u\left(t-\frac{W_{a}}{v_{p}}-\frac{x_{t}}{v_{p}}\right)\right\}\right] \\
& N_{s}(t)=\frac{\eta P_{i n}\left(M_{0}-1\right)}{h v}\left[\left[1-e^{-\alpha v_{p} t-\alpha x_{t}}\right]\left\{u\left(t-\frac{x_{t}}{v_{p}}\right)-u\left(t-\frac{x_{a}}{v_{p}}\right)\right\}\right. \\
& +\left[1-e^{-\alpha W_{a}}\right]\left\{u\left(t-\frac{x_{a}}{v_{p}}\right)-u\left(t-\frac{x_{a}}{v_{p}}-\frac{x_{a}}{v_{n}}\right)\right\} \\
& \left.-\left[1-e^{-\alpha\left(v_{p} t-\frac{x_{a} v_{p}}{v_{n}}-x_{t}\right)}\right]\left\{u\left(t-\frac{x_{t}}{v_{p}}-\frac{x_{a}}{v_{n}}\right)-u\left(t-\frac{x_{a}}{v_{p}}-\frac{x_{a}}{v_{n}}\right)\right\}\right] *\left[e_{h} e^{-t e_{h}}\right] * \frac{e^{\frac{-t}{\left(M_{0}-1\right) t_{m}}}}{\left(M_{0}-1\right) t_{m}}
\end{aligned}
$$

where $P_{i n}$ is the incident optical power (W), hv is the photon energy $(J), \eta$ is the external quantum efficiency, $\alpha$ the absorption coefficient $\left(m^{-1}\right), M_{0}$ the gain of the APD, $M_{0} \cdot t_{m}$ is the avalanche built up time (s), $x_{m}, x_{t}, x_{a}, W$ the distances described in Figure 2.3, $W_{a}$ the length of absorption region and where $W_{a}=x_{a}-x_{t}$ and $e_{h}$ is the emission rate of hole trapping. It is important to note that the symbol $*$ refers to the convolution operation in time. The function $u(t)$ is a unitary step function that is defined in (2.5).

$$
u(t)= \begin{cases}1 & t \geq 0 \\ 0 & t<0\end{cases}
$$


The resistive, capacitive and inductive parasitics can be modelled as a circuit shown in Figure 2.4.

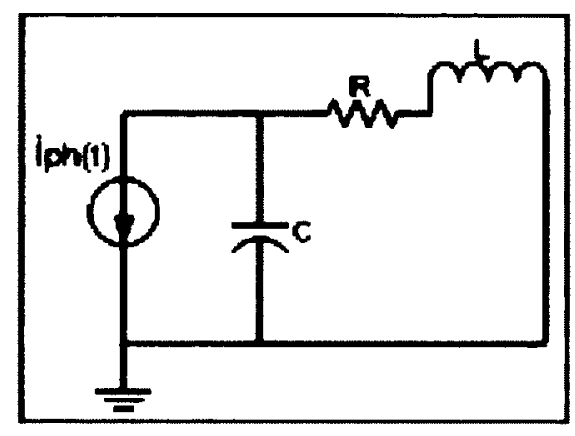

Figure 2.4: Resistive, capacitive and inductive parasitics in a circuit [2]

Note that the $i_{p h}(t)$ is the photogenerated current from (2.1). The overall output of this APD model is the current that is flowing through the inductor and resistor.

The next subsection will review the time domain model of SAGCM-APD [3] .

\subsubsection{Review of SAGCM APD Time Domain Model}

An improvement was made to the SAM-APD by adding additional grading and charge layers. Note that for this device the length of the absorption layer is given as $W_{a}$, the length of charge layer is $x_{c}$, the length of grading layer is $x_{g}$ and multiplication layer $x_{m}$

The photogenerated current for this device is again the superposition of primary 
and secondary holes and electrons given as (2.6):

$$
i_{p h}(t)=\frac{q}{W}\left(v_{n} N(t)+v_{p} P(t)+v_{n} N_{s}(t)+P_{s}(t)\right)
$$

Note that $N(t), P(t)$ are the function for primary mobile electrons and holes, $N_{s}(t), P_{s}(t)$ are the secondary mobile electrons and holes.

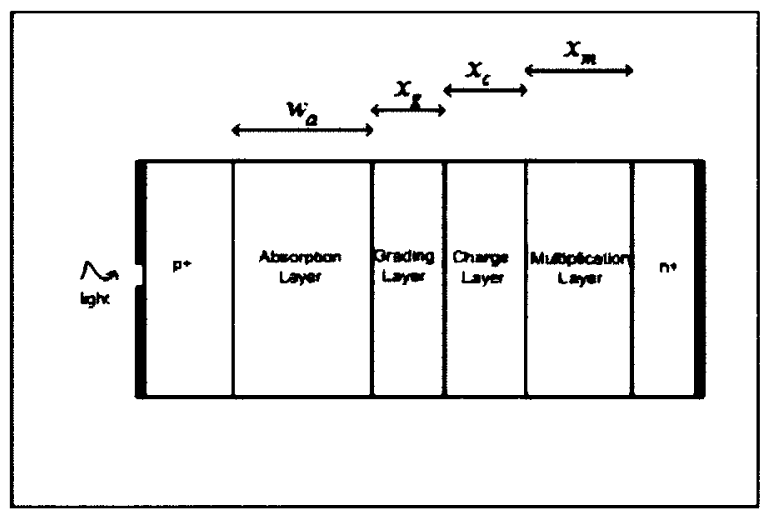

Figure 2.5: Basic structure of SAGCM APD from [3]

The three main effects modelled in the SAGCM APD model [3] are the avalanche buildup, the carrier transit time and the RLC parasitics. The effects are present in the primary mobile electrons and holes as shown in (2.7) and (2.8).

$$
\begin{gathered}
N(t)=\frac{\eta P_{0}}{h v}\left\{\left[1-e^{-\alpha W_{a}}\right] \cdot\left[u(t)-u\left(t-\frac{x_{t}+x_{m}}{v_{n}}\right)\right]+\left[1-e^{-\alpha\left(x_{t}+x_{m}+W_{a}\right)+\alpha v_{n} t}\right] .\right. \\
\left.\left[u\left(t-\frac{x_{t}+x_{m}}{v_{n}}\right)-u\left(t-\frac{x_{t}+x_{m}+W_{a}}{v_{n}}\right)\right]\right\}
\end{gathered}
$$




$$
P(t)=\frac{\eta P_{i n}}{h v}\left[e^{-\alpha v_{p} t}-e^{-\alpha W_{a}}\right] \cdot\left[u(t)-u\left(t-\frac{W_{a}}{v_{p}}\right)\right]
$$

The secondary mobile holes and electrons are shown in (2.9) and (2.10).

$$
\begin{aligned}
& P_{s}(t)=\frac{\eta P_{i n}}{h v}\left(M_{0}-1\right)\left\{e^{-\alpha W_{a}} \cdot\left[e^{\alpha x_{t}+\alpha v_{n} t}-1\right] \cdot\left[u\left(t-\frac{x_{t}}{v_{n}}\right)-u\left(t-\frac{x_{t}+W_{a}}{v_{n}}\right)\right]+\left[1-e^{-\alpha W_{a}}\right]\right. \\
& {\left[u\left(t-\frac{x_{t}+W_{a}}{v_{n}}\right)-u\left(t-\frac{x_{t}}{v_{n}}-\frac{x_{t}+W_{a}}{v_{p}}\right)\right]+\left[1-e^{-\alpha\left(x_{t}+W_{a}\right)} \cdot e^{\left.-\alpha \frac{v_{n}}{v_{p}\left(x_{t}+W_{a}\right)+\alpha v_{n} t}\right]}\right.} \\
& \left.\quad\left[u\left(t-\frac{x_{t}}{v_{n}}-\frac{x_{t}+W_{a}}{v_{p}}\right)-u\left(t-\frac{x_{t}+W_{a}}{v_{n}}-\frac{x_{t}+W_{a}}{v_{p}}\right)\right]\right\} *\left[\frac{e^{-\frac{t}{\left(M_{0}-1\right) \tau_{m}}}}{\left(M_{0}-1\right) \tau_{m}}\right] \\
& N_{s}(t)=\frac{\eta P_{0}}{h v}\left(M_{0}-1\right)\left\{\left[e^{-\alpha\left(x_{t}+W_{a}-v_{n} t\right)}-e^{-\alpha W_{a}}\right]\left[u\left(t-\frac{x_{t}}{v_{n}}\right)-u\left(t-\frac{x_{t}+x_{m}}{v_{n}}\right)\right]\right. \\
& +\left[e^{-\alpha\left(x_{t}+W_{a}\right)+\alpha v_{n} t}-e^{-\alpha\left(x_{t}+x_{m}+W_{a}\right)+\alpha v_{n} t}\right]\left[u\left(t-\frac{x_{t}+x_{m}}{v_{n}}\right)-u\left(t-\frac{x_{t}+W_{a}}{v_{n}}\right)\right] \\
& \left.+\left[1-e^{-\alpha\left(x_{t}+x_{m}+W_{a}\right)+\alpha v_{n} t}\right]\left[u\left(t-\frac{x_{t}+W_{a}}{v_{n}}\right)-u\left(t-\frac{x_{t}+x_{m}+W_{a}}{v_{n}}\right)\right]\right\} *\left[\frac{e^{-\frac{t}{\left(M_{0}-1\right) \tau_{m}}}}{\left(M_{0}-1\right) \tau_{m}}\right]
\end{aligned}
$$

Note that all the variables within this model are shared by the parameters of the previous model.

The resistive, capacitive and inductive parasitics can be represented in the same way as done in the previous model and shown in Figure 2.6. The next two sections will review the frequency APD models of each structure. 


\subsection{Frequency APD Model}

\subsubsection{SAM APD}

The frequency domain APD model [2] as given below in (2.11) is achieved by taking the Fourier transform of (2.1).

$\frac{i_{p h}(\omega)}{i_{p h}(0)}=\frac{1}{M_{0}\left(1-e^{-\alpha W_{a}}\right)} \frac{1}{\left(1-\omega^{2} L C+j \omega R C\right)}\left[T_{n}(\omega)+T_{p}(\omega)+\frac{T_{n s}(\omega)}{\left(1+j \omega\left(M_{0}-1\right) t_{m}\right)\left(1+\frac{j \omega}{e_{h}}\right)}\right]$

where:

$$
\begin{gathered}
T_{n}(\omega)=\frac{v_{n}}{W}\left[\left[\frac{1-e^{-j \omega \frac{W_{a}}{v_{n}}}}{j \omega}\right]-e^{-\alpha W_{a}}\left[\frac{1-e^{\frac{-j \omega W_{a}}{v n}+\alpha W_{a}}}{j \omega-\alpha v_{n}}\right]\right] \\
T_{p}(\omega)=\frac{v_{p}}{W}\left[\left[\frac{1-e^{-\alpha W_{a}}}{j \omega}\right]+\left[\frac{\frac{j \omega}{e_{h}}+e^{\frac{-j \omega x x_{x} t}{v_{p}}}}{1+\frac{j \omega}{\epsilon_{h}}}\right]\left[1-e^{-\frac{-j \omega W_{a}}{v_{p}}}-\alpha W_{a}\right]\left[\frac{1}{j \omega+\alpha v_{p}}-\frac{1}{j \omega}\right]\right]
\end{gathered}
$$

The secondary electron number of carriers, $T_{n s}(\omega)$, in the frequency domain is given as,

$$
T_{n s}(\omega)=\frac{v_{n}\left(M_{0}-1\right)}{W}\left[e^{\frac{-j \omega x_{t}}{v_{p}}}\left[1-e^{\frac{-j \omega x_{a}}{v_{n}}}\right]\left[1-e^{\frac{-j \omega W_{a}}{v_{p}}-\alpha W_{a}}\right]\left[\frac{1}{j \omega+\alpha v_{p}}-\frac{1}{j \omega}\right]\right]
$$

Note that $\omega$ is the angular frequency $(\mathrm{rad} / \mathrm{s})$ while $T_{n}(\omega), T_{p}(\omega)$ are the primary electron and hole carrier transit time and $T_{n s}(\omega)$ is the secondary electron carrier transit time in the frequency domain representation. 
The DC solution of the frequency APD model, $i_{p h}(0)[2]$ is given as (2.15).

$$
i_{p h}(0)=P D_{e f f} M_{0}\left[1-e^{-\alpha W_{a}}\right]
$$

where:

$$
P D_{e f f}=\frac{\eta P_{i n}}{h v}
$$

The next section will review the SAGCM-APD frequency macromodel.

\subsubsection{SAGCM-APD}

The three main effects modelled in the frequency domain SAGCM-APD physical model [3] are the avalanche buildup, the carrier transit time and the RLC parasitics. Using [2] and [3], the frequency APD model was developed by finding the number of carriers that cross the depletion region as shown in (2.17):

$$
H(\omega)=\frac{q}{D}\left(v_{n}\left(N(\omega)+N_{s}(\omega)\right)+v_{p}\left(P(\omega)+P_{s}(\omega)\right)\right)
$$

where $q$ is the electric charge (C), D is the length of the whole depletion region, $v_{n}\left(\frac{m}{s}\right), v_{p}\left(\frac{m}{s}\right)$ electron and hole saturated drift velocity, $N(\omega), P(\omega)$ are the number of mobile primary electrons and holes as given in (2.18), (2.19), $N_{s}(\omega), P_{s}(\omega)$ are the 
number of secondary mobile electrons and holes as shown in $(2.20),(2.21)$ :

$$
\begin{gathered}
N(\omega)=\frac{\eta P_{0}}{h v}\left\{e^{\frac{-j \omega\left(x_{t}+x_{m}\right)}{v n}}\left[e^{-a \cdot W_{a}}-e^{\frac{-j \omega W_{a}}{v_{n}}}\right]\left(\frac{1}{j \omega}+\frac{1}{a v_{n}-j \omega}\right)+\frac{1}{j \omega}\left(1-e^{a \cdot W_{a}}\right)\right\} \\
P(\omega)=\frac{\eta P_{0}}{h v}\left\{\frac{1}{a \cdot v_{p}+j \omega}\left[1-e^{-a \cdot W_{a}-\frac{j \omega W_{a}}{v_{p}}}\right]-\frac{e^{-a W_{a}}}{j \omega}\left[1-e^{\frac{-j \omega W_{a}}{v p}}\right]\right\} \\
N_{s}(\omega)=\frac{\eta P_{0}}{h v}\left(\frac{1}{j w}+\frac{1}{a \cdot v_{n}-j \omega}\right)\left[e^{\frac{-j \omega W_{a}}{v_{n}}}-e^{-a \cdot W_{a}}\right] e^{\frac{-j \omega x_{t}}{v_{n}}} \cdot\left[1-e^{\frac{-j \omega x_{m}}{v_{n}}}\right] \frac{M-1}{1+j \omega(M-1) \tau_{m}} \\
P_{s}(\omega)=\frac{\eta P_{0}}{h v} e^{\frac{-j \omega x_{t}}{v_{p}}}\left(\frac{1}{j \omega}+\frac{1}{a \cdot v_{n}-j \omega}\right) \cdot\left(1-e^{\frac{-j \omega\left(x_{t}+W_{a}\right)}{v_{p}}}\right)\left[e^{\frac{-j \omega W_{a}}{v_{n}}}-e^{-a \cdot W_{a}}\right] \frac{M-1}{1+j \omega(M-1) \tau_{m}}
\end{gathered}
$$

where $\alpha$ is the absorption coefficient $\left(m^{-1}\right), M$ is the APD gain, $x_{t}$ is the length of total thickness of the grading and charge layer.

The overall transfer function that includes the parasitics is given as, (2.22).

$$
\frac{I_{p h}(\omega)}{I_{p h}(0)}=H(\omega) H_{p a r}(\omega)
$$

where,

$$
I_{p h}(0)=P D_{e f f} M_{0}\left[1-e^{-\alpha W_{a}}\right]
$$

and the parasitic parameter is given below: 


$$
H_{p a r}(\omega)=\frac{1}{1+j \omega R C-\omega^{2} C L}
$$

Note that $R, C$ and $L$ are the values of the resistor, capacitor and inductor that model the parasitics of the device.

\subsubsection{APD Model Stability}

In this subsection the definition of stability is reviewed and how it applies to the APD models.

In general, a system's impulse response is said to be stable if and only if the transfer function $h(t)$ converges to zero as $t \rightarrow \infty$. This is characterized in the frequency domain by checking if the poles of the transfer are on the left hand side of the plane of the Laplace domain [9].

The APD frequency response of the term $G(\omega)=j \omega-a v_{n}, N(\omega)$ 's denominator acts like an unstable pole if it is separated from its numerator. This instability occurs because the pole is $\alpha v_{n}>0$ as shown below.

$$
N(\omega)=\frac{v_{n}}{W}\left[\left[\frac{1-e^{-j \omega \frac{W_{a}}{v_{n}}}}{j \omega}\right]-e^{-\alpha W_{a}}[\underbrace{\frac{1-e^{\frac{-j \omega W_{a}}{v_{n}}+\alpha W_{a}}}{j \omega-\alpha v_{n}}}_{G(\omega)}]\right]
$$


$N(\omega)$ 's time domain representation is shown below.

$$
N(t)=\frac{\eta P_{i n}}{h v}\left[1-e^{-\alpha W_{a}+\alpha v_{n} t}\right] \underbrace{\left\{u(t)-u\left(t-\frac{W_{a}}{v_{a}}\right)\right\}}_{\text {delay }}
$$

If $t \rightarrow \infty$, then the terms $u(t)-u\left(t-\frac{W_{a}}{v_{a}}\right)$ will go to zero and make $N(t)$ zero. But if the delay is to be removed from $N(t)$, the positive exponential shown in (2.26) will diverge to $\infty$ as $t \rightarrow \infty$. Therefore $N(t)$ is stable due to the presence of the delays [9]. As a result, the terms $u(t)-u\left(t-\frac{W_{a}}{v_{a}}\right)$ cannot be separated from the growing exponential.

\subsection{Review of Time Domain APD Modelling Tech- niques}

In this section, two time domain APD macromodels will be reviewed. The first method used is the traditional convolution. The process of convolution will be reviewed in the next section. 


\subsubsection{Review of Traditional Convolution and the FFT-APD Model}

Inversion of the frequency domain models above provide a time domain impulse response for the APD. In order to find the APD response to an arbitary input the impulse current carrier equations must be convoluted with the optical input as shown in (2.27) and (2.28).

$$
\begin{gathered}
i_{\text {out }}(t)=h(t) * P_{\text {in }}(t) \\
=\int_{-\infty}^{\infty} h(\tau) P_{\text {in }}(t-\tau) d \tau
\end{gathered}
$$

A solution to (2.28) can be implemented using an FFT algorithm for which the convolution in the time domain becomes a multiplication in the frequency domain. [10]. However, the FFT algorithm is a numerical technique that assumes the input signal is periodic and can be cumbersome and time consuming when used in a time domain simulator. In order to use FFT algorithm, a frequency response must be solved such that the discrete frequency points meet the Nyqvist Criterion and periodicity must be assumed. For this thesis a general FFT based signal processing element was available in the time domain simulator OptiSPICE [7]. This element uses a numerical representation of the filter in the frequency domain and implements an FFT based "element" that provides a time domain representation of the signal filtering. The element requires a signal bandwidth to be specified and is typically very slow as an FFT/IFFT 
has to be used at every time step. The APD model utilizing this FFT/IFFT element is denoted as an FFT-APD.

\subsubsection{Review of Circuit Equivalent APD}

A second APD macromodel approach, called Circuit Equivalent APD (CE-APD), was developed for a Separate Absorption Grading Charge Multiplication Avalanche Photodiode SAGCM-APD by [3]. In order to reproduce the time domain simulation of SAGCM-APD, the frequency SAGCM-APD physical model must be reviewed. The SAGCM-APD is an APD that was developed to improve performance of an APD [11] by adding additional grading and charge layers while separating the absorption layer from the multiplication layer as shown in Figure 2.6. Note that the length of the absorption layer is $W_{a}$, the length of charge layer is $x_{c}$, the length of grading layer is $x_{g}$ and multiplication layer $x_{m}$.

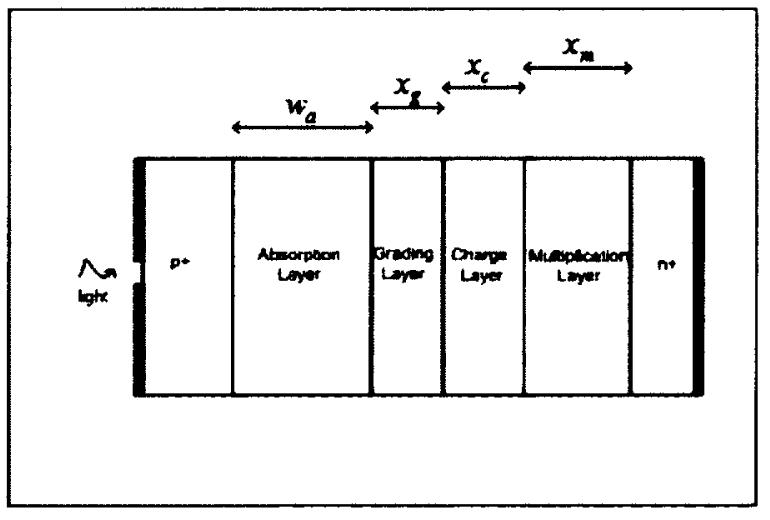

Figure 2.6: Basic structure of SAGCM-APD [3] 
The three main effects modelled in the frequency domain SAGCM-APD physical model [3] are the avalanche buildup, the carrier transit time and the RLC parasitics.

The SAGCM-APD frequency model is transformed into an equivalent circuit. The CE-APD macromodel applies superposition to break the SAGCM-APD model into a group of subsystems as shown in Figure 2.7 .

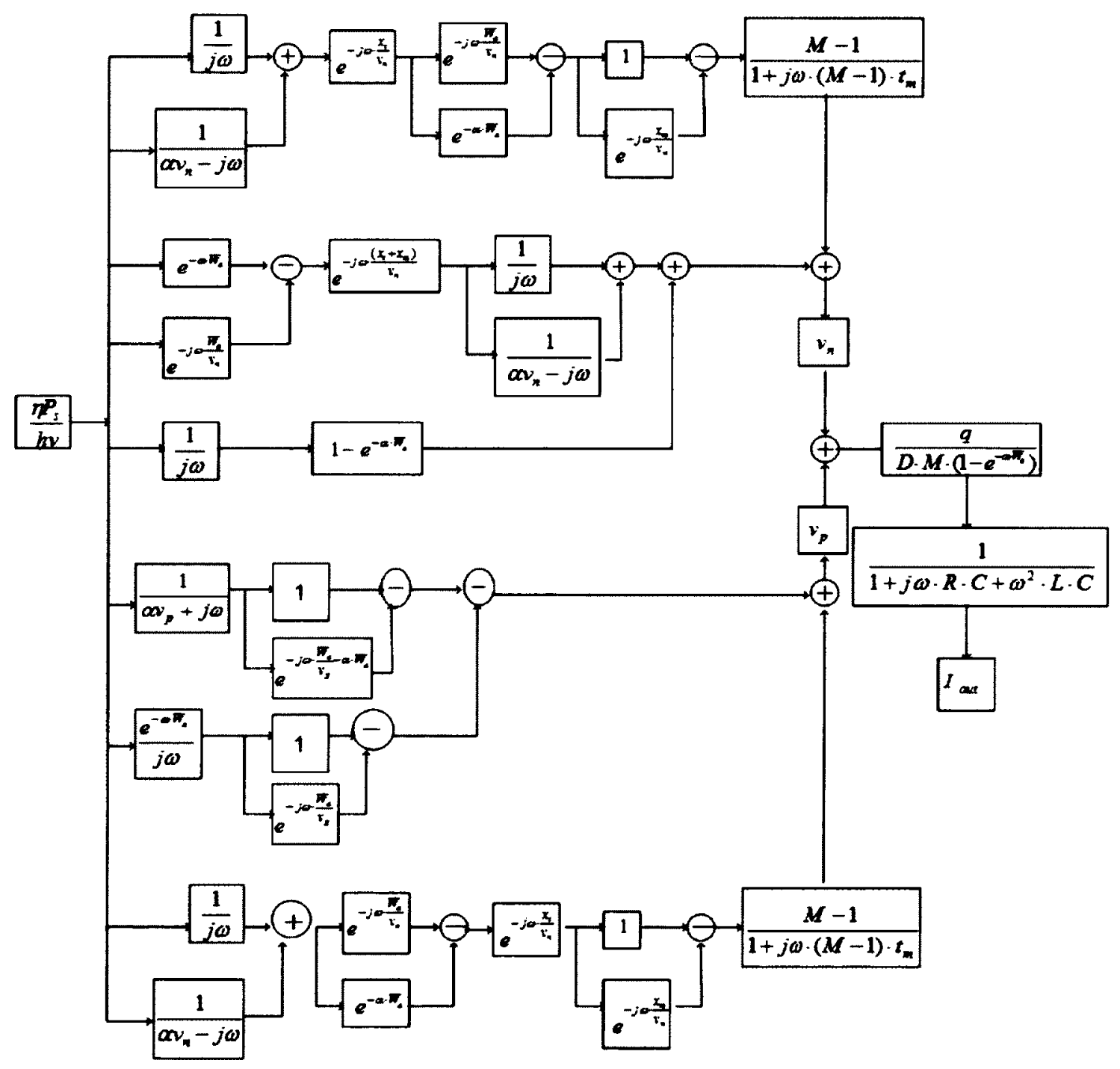

Figure 2.7: Basic block diagram of SAGCM APD [3] 
There are 6 different subsystem blocks that compose the APD system. Note that $I_{\text {in }}(\omega)=\frac{\eta P}{h v}$ is the input to the system, $I_{\text {out }}(\omega)$ or $I_{\text {out }}$ is the output current and $x$ is any constant variable in the equation. The first block is given in (2.29) and Figure 2.8 .

$$
I_{\text {out }}(\omega)=e^{-j \omega x} I_{\text {in }}(\omega)
$$

This block can be synthesized into an equivalent circuit delay as shown in the Figure 2.8 .

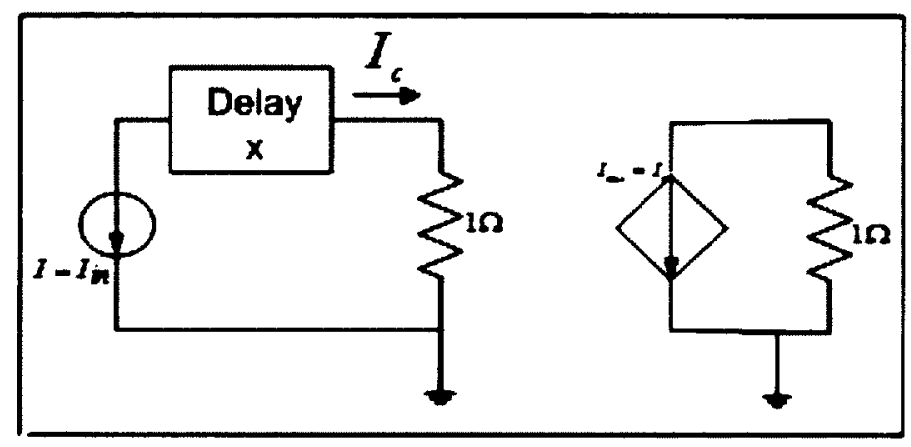

Figure 2.8: Synthesis of the frequency equivalent delay block

The second subsystem block is given in (2.30) and Figure 2.9, represents an ideal integrator in the frequency domain. An approximate integrator can be synthesized with a capacitor connected to a current source where the voltage is the output as shown in Figure 2.9. Note that there is a large resistor in parallel to provide a DC path to ground. Therefore the subsystem (2.30) and the circuit in Figure 2.9 will not 
match at low frequency. In the time domain, a small DC offset maybe noticed.

$$
I_{\text {out }}(\omega)=\frac{1}{j \omega} I_{\text {in }}(\omega)
$$

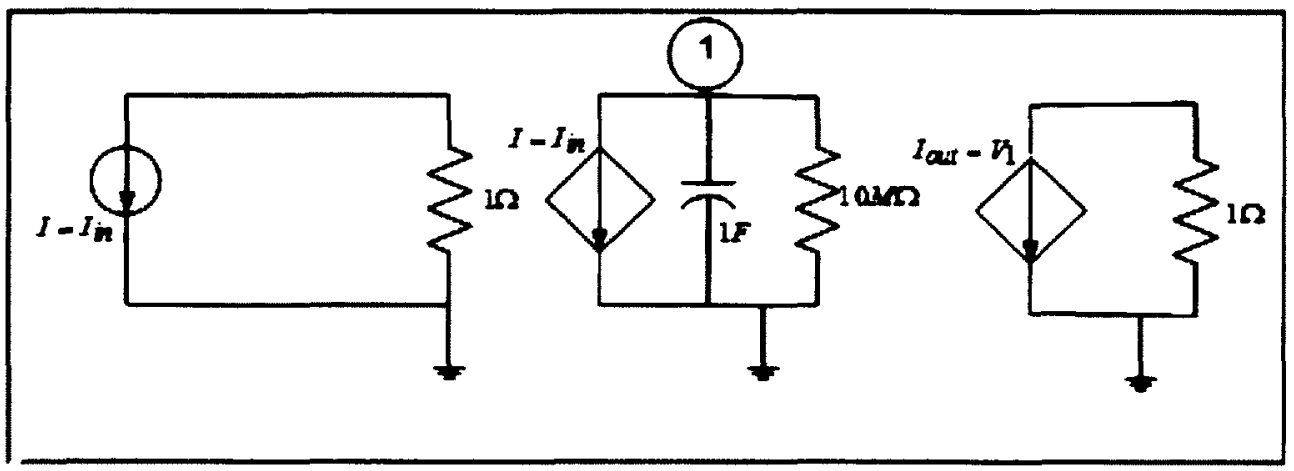

Figure 2.9: Synthesis of the frequency equivalent integrator block

The third subsystem block can be represented as a left hand pole filter as shown in (2.31) and Figure 2.10. The transfer function is treated as an admittance where the input current is treated as a voltage using a current controlled voltage source.

$$
I_{\text {out }}(\omega)=\frac{1}{x+j \omega} I_{\text {in }}(\omega)
$$

The left hand pole can be synthesized into an inductor and resistor, where the value of inductor is set to unity and the resistor is set to value of $x$ as shown in Figure 2.10. 


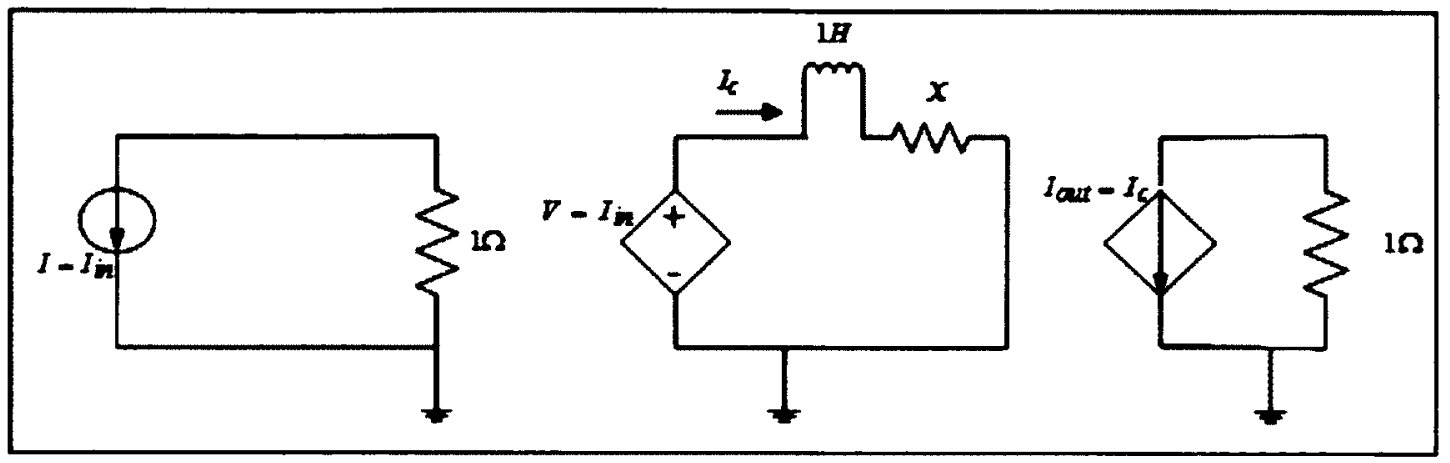

Figure 2.10: Synthesis of the frequency stable RL reasonator block

The fourth subsystem block represented as a right hand pole filter as shown in (2.32) and Figure 2.11.

$$
I_{\text {out }}(\omega)=\frac{-1}{j \omega-x} I_{\text {in }}(\omega)
$$

The block (2.32) was implemented in a similar fashion to the previous block with the difference being a negative resistor value shown in Figure 2.11. Note that the negative resistor is a behavioural circuit element that represents the active portion of the APD physical model. And this allows the block to be used to simulate circuits such as oscillators.

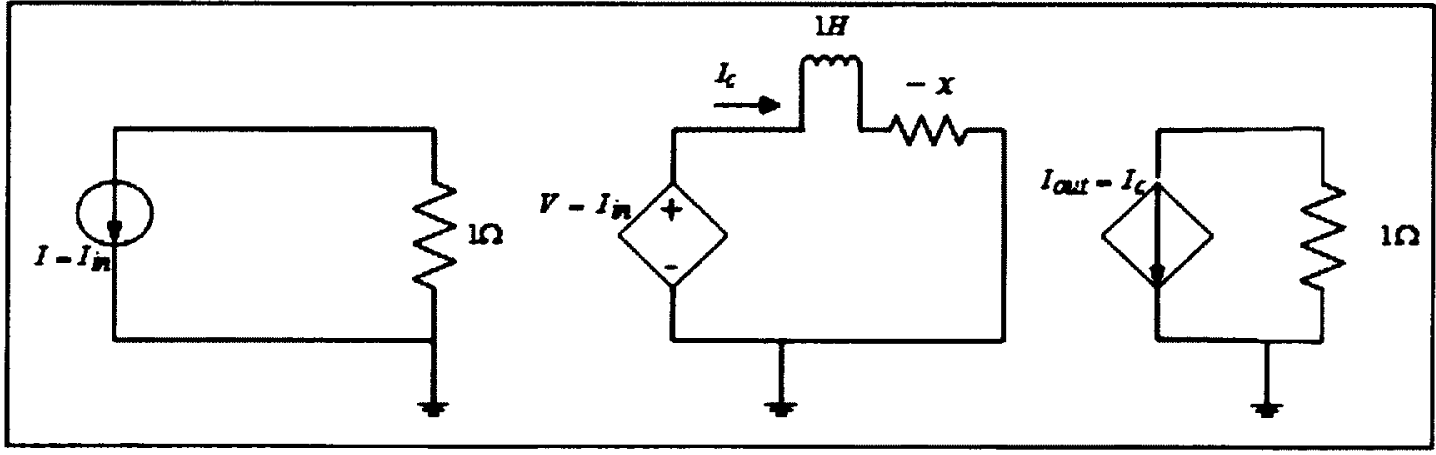

Figure 2.11: Synthesis of the frequency equivalent unstable RL resonator block 
The fifth block as given in (2.33) represented a simple constant gain circuit as shown in Figure 2.12.

$$
I_{\text {out }}(\omega)=x I_{\text {in }}(\omega)
$$

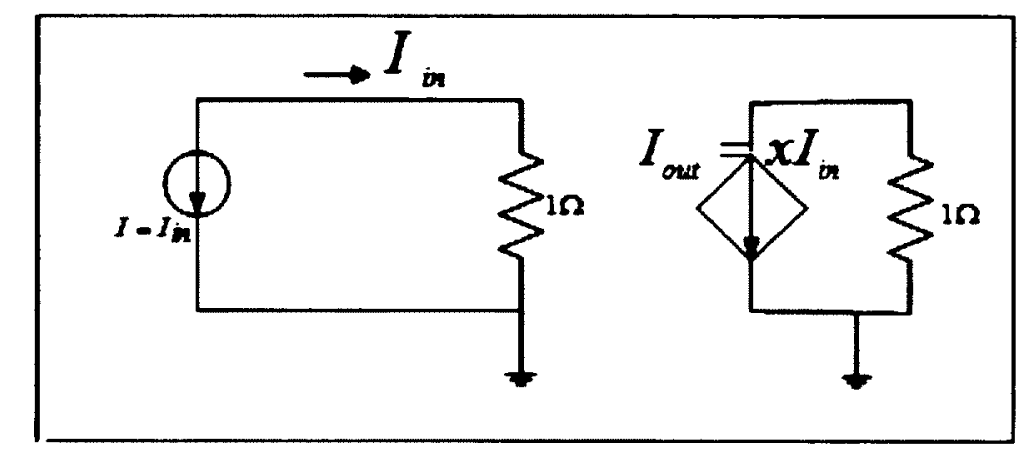

Figure 2.12: Synthesis of the constant gain block

For completeness, the sixth block as given in (2.34) is a differentiator in frequency domain.

$$
I_{\text {out }}(\omega)=j \omega I_{\text {in }}(\omega)
$$

The differentiator block can be synthesized into an inductor where the input is a current source and the output is the voltage as seen in Figure 2.13. 


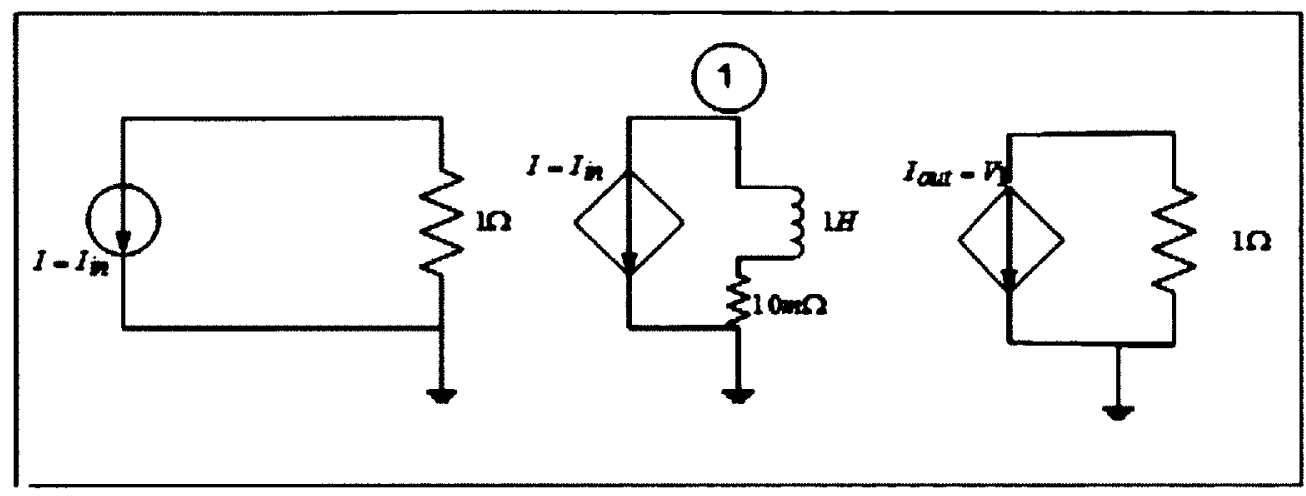

Figure 2.13: Synthesis of the frequency equivalent differentiator block

Note that there is a small behavioural series resistor element to insure that the inductor does not short the input at DC, during circuit simulation. Although the sixth block is not used in SAGCM, it will be applied in the next chapter and is included here.

The synthesized circuits can easily be cascaded using controlled sources to isolate each stage. The addition and subtraction operation is performed by connecting the controlled sources in parallel to a $1 \Omega$ resistor.

The development of the CE-APD model is the generation of a netlist which can easily be included in a netlist as a sub-circuit for the simulation of a circuit. This macromodel contains the device model information, which can easily be changed and re-simulated. The speed and accuracy of the model are determined by the circuit simulator being used. The only loss in accuracy is due to approximation of the integrator subsystem and the differentiator subsystem for low frequency signals. However, 
this physical model is plagued with stability problems and thus is impractical. This problem will be illustrated in the next chapter and a solution proposed. 


\section{Chapter 3}

\section{QC-APD Macromodel}

This chapter will discuss the Quasi Circuit APD Macromodel (QC-APD) and its control of accuracy and speed using the $N_{\text {seg }}$ parameter. Note that $N_{\text {seg }}$ refers to an internal parameter for QC-APD that represents the number of discrete segments of $N(t)$

The first section will present the origin of QC-APD. The second section will present the accuracy and simulation processing time of QC-APD using $N_{\text {seg }}$ parameter.

\subsection{The Origin of QC-APD Macromodel}

The QC-APD macromodel originated from a model implemented in OptiSPICE which was independently developed but essentially identical to the CE-APD macromodel. Both the OptiSPICE and the CE-APD models exhibited stability problems. In order 
to present this problem, a time domain simulation example of SAGCM-APD will be presented in the first subsection using CE-APD macromodel. The second subsection will present the creation of QC-APD as a solution for CE-APD problem.

\subsubsection{Time Domain Simulation of SAGCM-APD using CE- APD Macromodel}

An example of a APD simulation using the CE-APD was performed by finding the step input response of the SAGCM-APD [3] with a simple linear resistive load of $1 \Omega$ as shown in Figure 3.1.

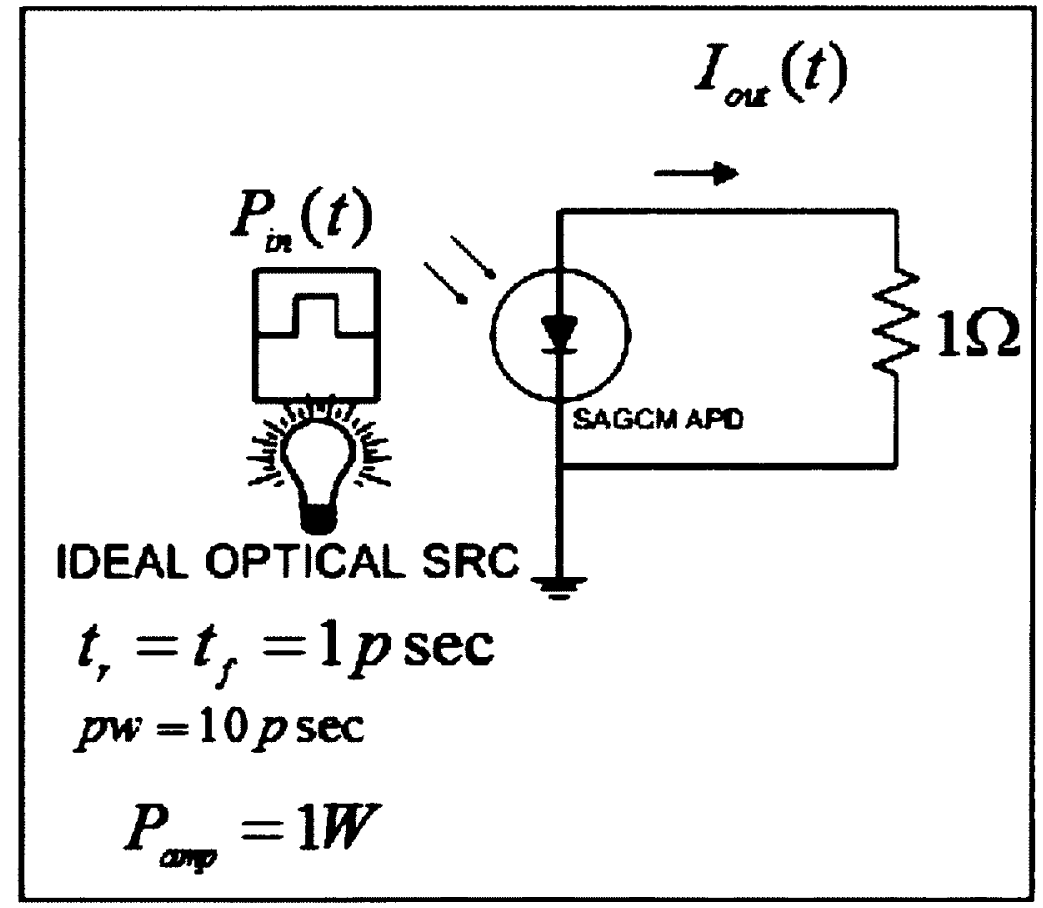

Figure 3.1: Circuit topology of SAGCM-APD that is connected to a $1 \Omega$ resistor 
The model parameters of the SAGCM-APD [3] are given in a Table 3.1 along with the simulation parameters, such as the input pulse rise time and fall time and the pulse width. In order to verify the simulation, the SAGCM-APD time domain response is simulated using FFT-APD.

\begin{tabular}{|c|c|}
\hline Parameter Names & Values \\
\hline \hline$P D_{\text {eff }}$ & 1 \\
\hline$W_{a}$ & $1.0 \mu \mathrm{m}$ \\
\hline$x_{g}$ & $150 \mathrm{~nm}$ \\
\hline$x_{c}$ & $150 \mathrm{~nm}$ \\
\hline$x_{m}$ & $200 \mathrm{~nm}$ \\
\hline$\alpha$ & $1.15 \mu m^{-1}$ \\
\hline $1 /\left(2 \pi \tau_{m}\right)$ & $120 \mathrm{GHz}$ \\
\hline$L$ & $0.3 \mathrm{nH}$ \\
\hline$C$ & $0.1 \mathrm{pF}$ \\
\hline$R$ & $60 \Omega$ \\
\hline$v_{p}$ & $6 \cdot 10^{6} \mathrm{~cm} / \mathrm{s}$ \\
\hline$v_{n}$ & $8 \cdot 10^{6} \mathrm{~cm} / \mathrm{s}$ \\
\hline$t_{r}, t_{f}$ & $1 \mathrm{psec}$ \\
\hline$p w$ & $10 \mathrm{psec}$ \\
\hline$M$ & 1 \\
\hline \multicolumn{2}{|c}{}
\end{tabular}

Table 3.1: Physical parameters of the SAGCM-APD [3]

The pulse response of the SAGCM CE-APD model and FFT-APD is shown in Figure 3.2. 


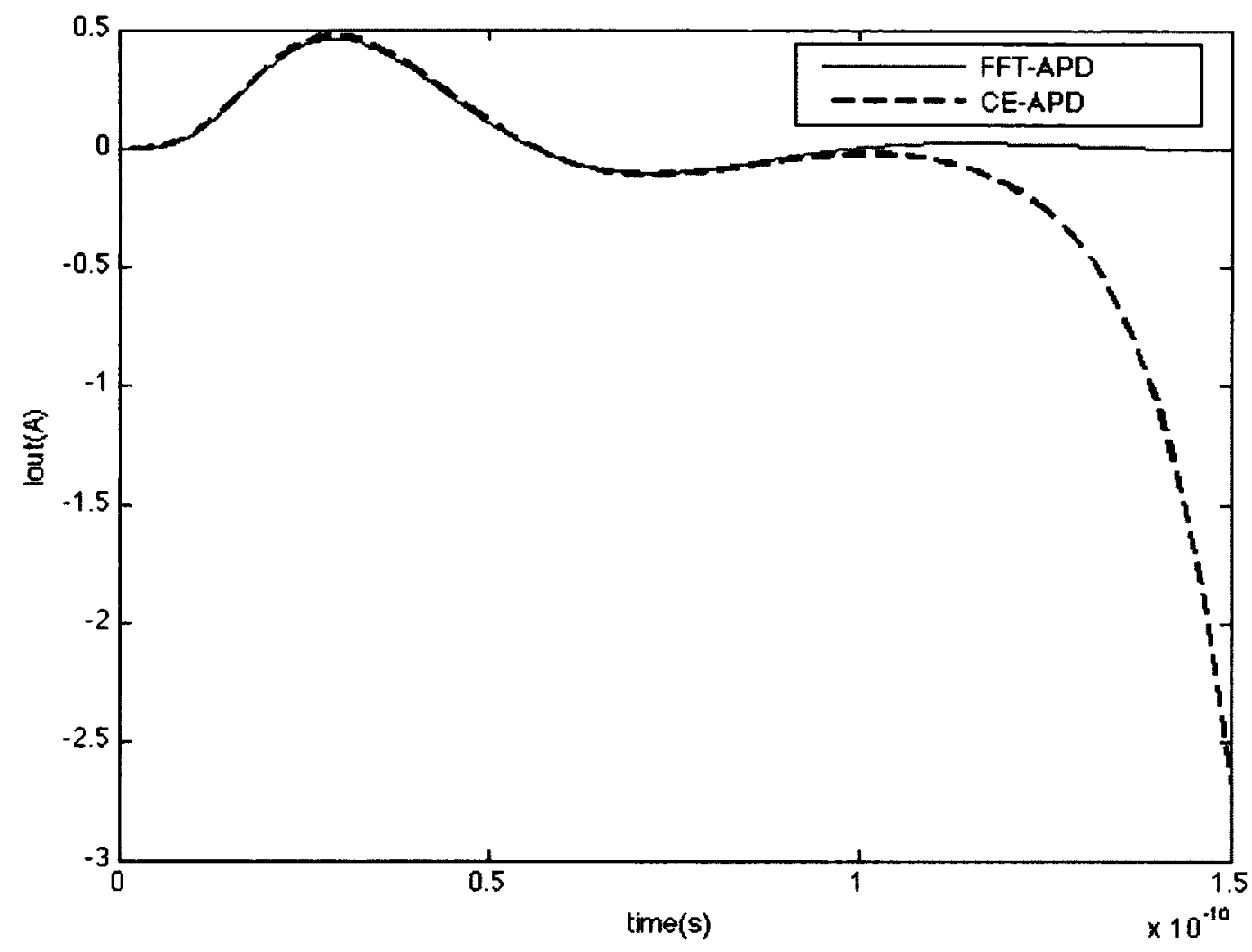

Figure 3.2: Time domain response of a trapezoidal pulse with a simple resistive load using the FFT-APD and CE-APD models

From Figure 3.2, it is noticed that the response of the CE-APD macromodel gives an unstable result. Both circuit simulators, HSPICE and OptiSPICE give warnings and indicate convergence problems. In order to determine the cause of this problem, the block diagram of the SAM-APD [2] is shown in Figure 3.3. 


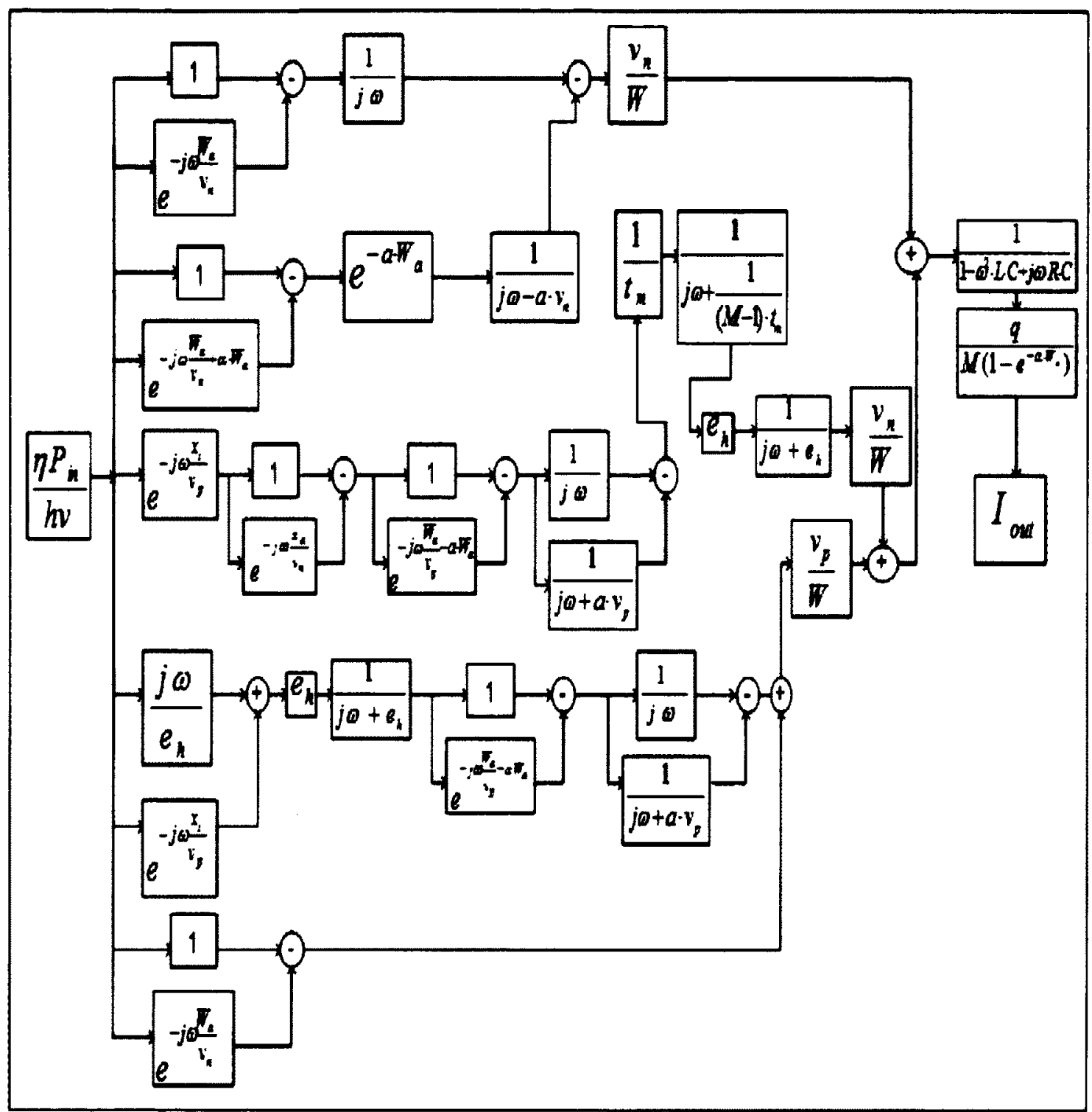

Figure 3.3: Block diagram of the SAM-APD [2]

The subsystem $\frac{1}{j \omega-\alpha v_{n}}$ as shown in Figure 3.3, has a right hand pole which is represented by the negative resistor. If the negative resistor dominates the response of the circuit or even that of the circuit block, the circuit becomes unstable. The next 
subsection will describe a solution for the negative resistance implementation.

\subsubsection{Fixing CE-APD Macromodel}

In order to fix the CE-APD's instability problem, $\frac{1}{a v_{n}-j \omega}$ must not be synthesized into an isolated circuit. The direct synthesis of $\frac{1}{a v_{n}-j \omega}$ is avoided using the method described below.

The $N(t)$ function is modelled as a time limited growing exponential, where its growth is limited by the delays as shown in (3.1).

$$
N(t)=\left[1-e^{-\alpha W_{a}+\alpha v_{n} t}\right]\left\{u(t)-u\left(t-\frac{W_{a}}{v_{p}}\right)\right\}
$$

This response needs to be represented as one entity to insure that the positive growing exponential stays bounded. In order to represent this system in a circuit, (3.1) will be solved and discretized into $\mathrm{N}$ segments where each segments will be a rectangular pulse as shown in Figure 3.4 . 


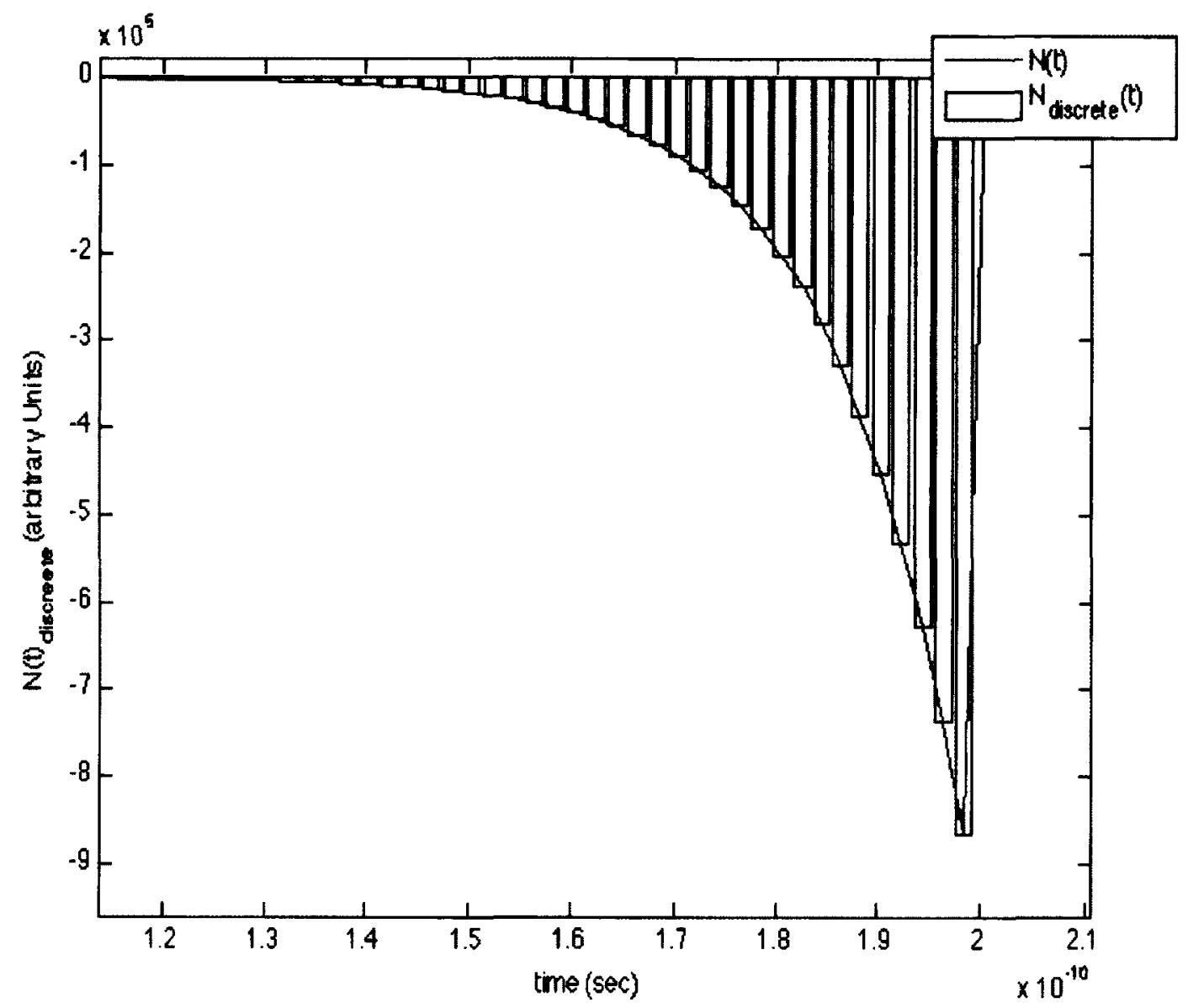

Figure 3.4: Discretizing of $N(t)$ function into rectangular pulses

Note that $N_{\text {discrete }}(t)$ refers to the discretized impulse response of $N(t)$ as shown in Figure 3.4. In order to find the current contribution due to $N_{\text {discrete }}(t)$ the convolution must be solved as

$$
I_{N(t)}=P_{\text {in }}(t) * N(t) \approx \int_{-\infty}^{\infty} P_{\text {in }}(t-\tau) \cdot N_{\text {discrete }}(\tau) d \tau
$$


The convolution operation can be between $P_{\text {in }}(t)$ and $N_{\text {discrete }}(t)$ as shown in Figure 3.5 .
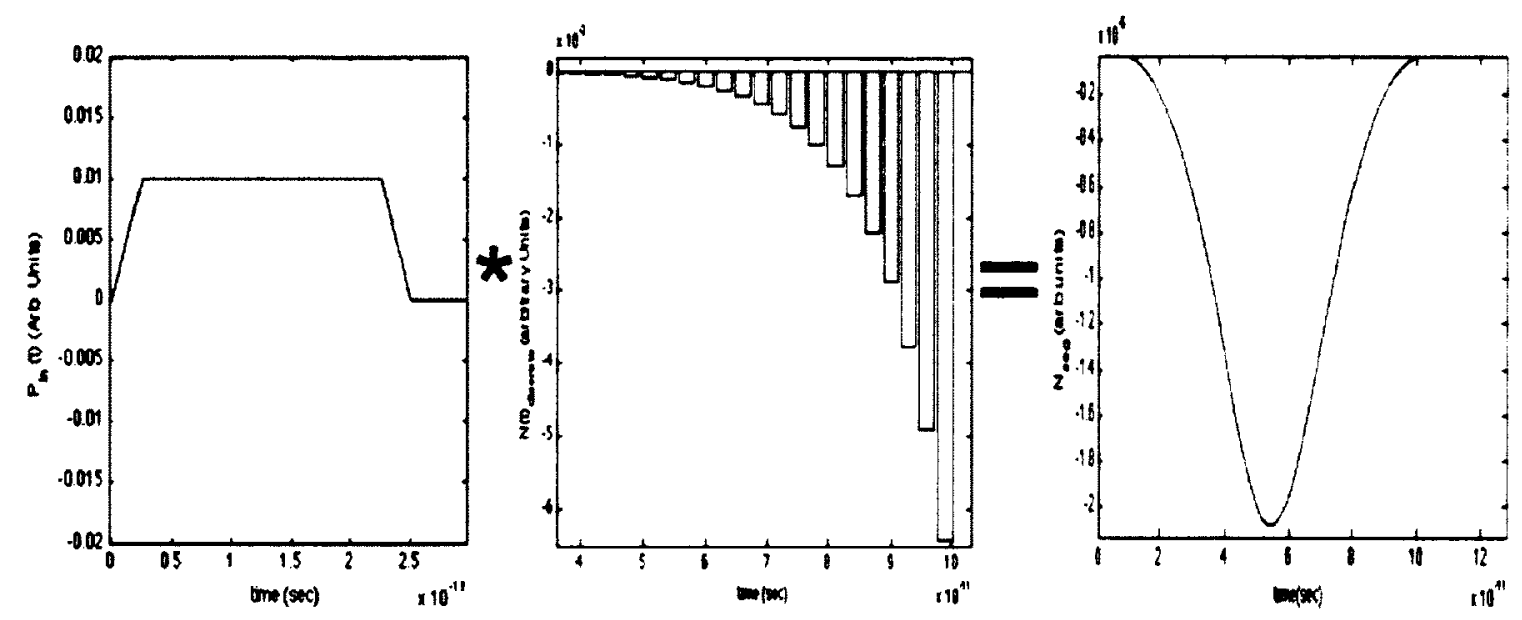

Figure 3.5: The time domain convolution between $P_{\text {in }}(t)$ and $N_{\text {discrete }}(t)$

In order to simplify the convolution operation, each individual segment of $N_{\text {discrete }}(t)$ will be analyzed independently as shown in Figure 3.6. Note that in this case $N_{\text {seg } 1}(t)$ is one of the segments from $N_{\text {discrete }}(t)$.
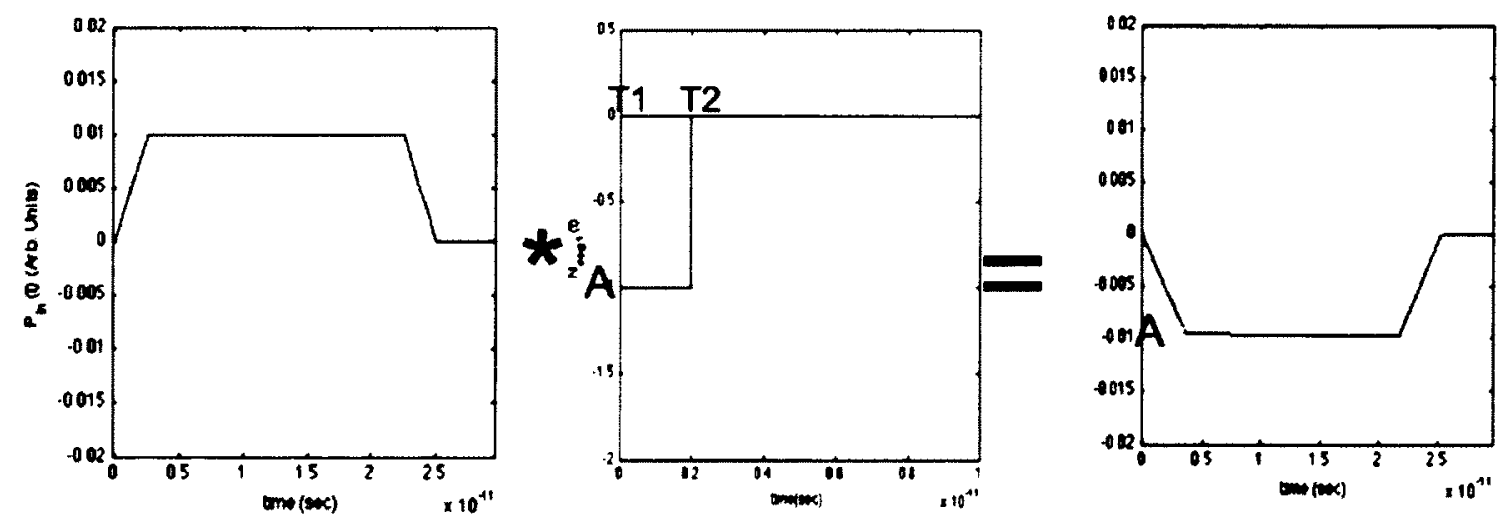

Figure 3.6: The time domain convolution between $P_{\text {in }}(t)$ and one discretized $N_{\text {seg } 1}(t)$ segment with amplitude A 
Let's compute the time domain convolution (3.2) using circuit representation.

In Figure 3.6, segment $A$ and $P_{i n}(t)$ are constant and are precomputed prior to circuit generation. In order to find the solution to Figure 3.6, (3.3) must be solved.

$$
P_{\text {in }}(t) * N_{\text {seg1 }}(t)=\int_{-\infty}^{\infty} P_{\text {in }}(t-\tau) \cdot N_{\text {seg1 }}(\tau) d \tau
$$

The equations of the $N_{\text {seg } 1}(t)$ is represented with a constant amplitude with delays as shown in (3.4)

$$
P_{i n}(t) * N_{\text {seg1 }}(t)=\int_{-\infty}^{\infty} P_{i n}(t-\tau) \cdot A(u(\tau-T 1)-u(\tau-T 2)) d \tau
$$

The next step is to separate (3.4) and get two different integration terms as shown in (3.5).

$$
P_{\text {in }}(t) * N_{\text {seg1 }}(t)=\underbrace{\int_{-\infty}^{\infty} P_{\text {in }}(t-\tau) \cdot A u(\tau-T 1) d \tau}_{\text {term } 1}-\underbrace{\int_{-\infty}^{\infty} P_{i n}(t-\tau) \cdot A u(\tau-T 2) d \tau}_{\text {term } 2}
$$

Note that $P_{i n}(t)$ and $A u(t-T 1)$ can be synthesized into a circuit and the integration operation can be be performed by a circuit integration. Thus term 1 can be represented by the circuit as shown in Figure 3.7. 


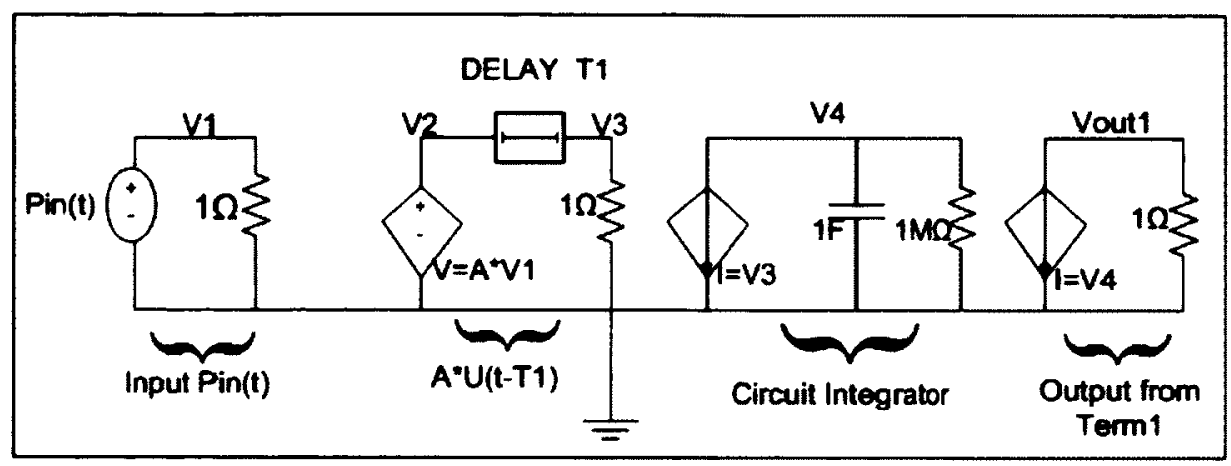

Figure 3.7: Circuit synthesized from term 1 in (3.5)

The similar approach can be used to synthesize term 2 as shown in Figure 3.8.

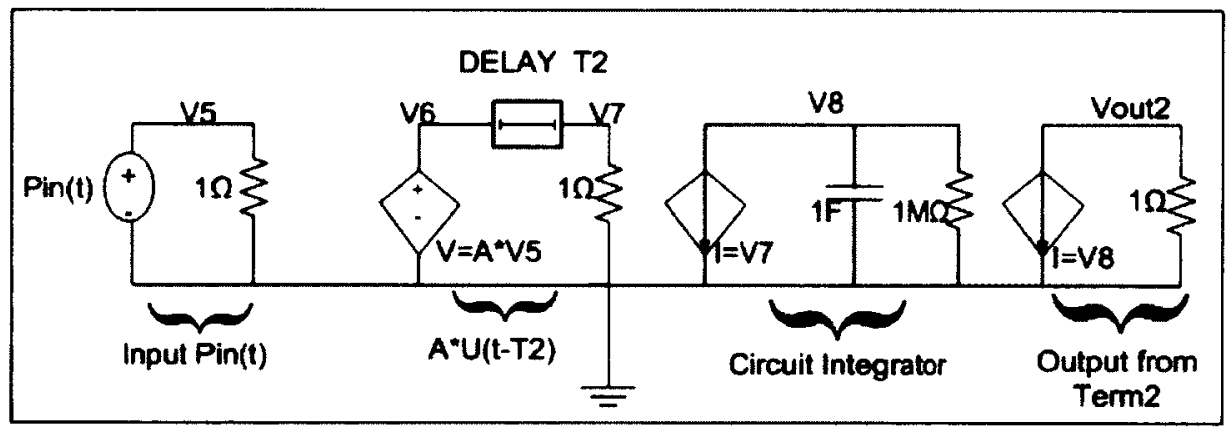

Figure 3.8: Circuit synthesized from term 2 in (3.5)

Note that $P_{i n}(t)$ is represented by a voltage source and $u(t-T 1)$ and $u(t-T 2)$ are represented by circuit delay elements.

Note Term 1 and Term 2 are subtracted from each other as shown in Figure 3.9. 


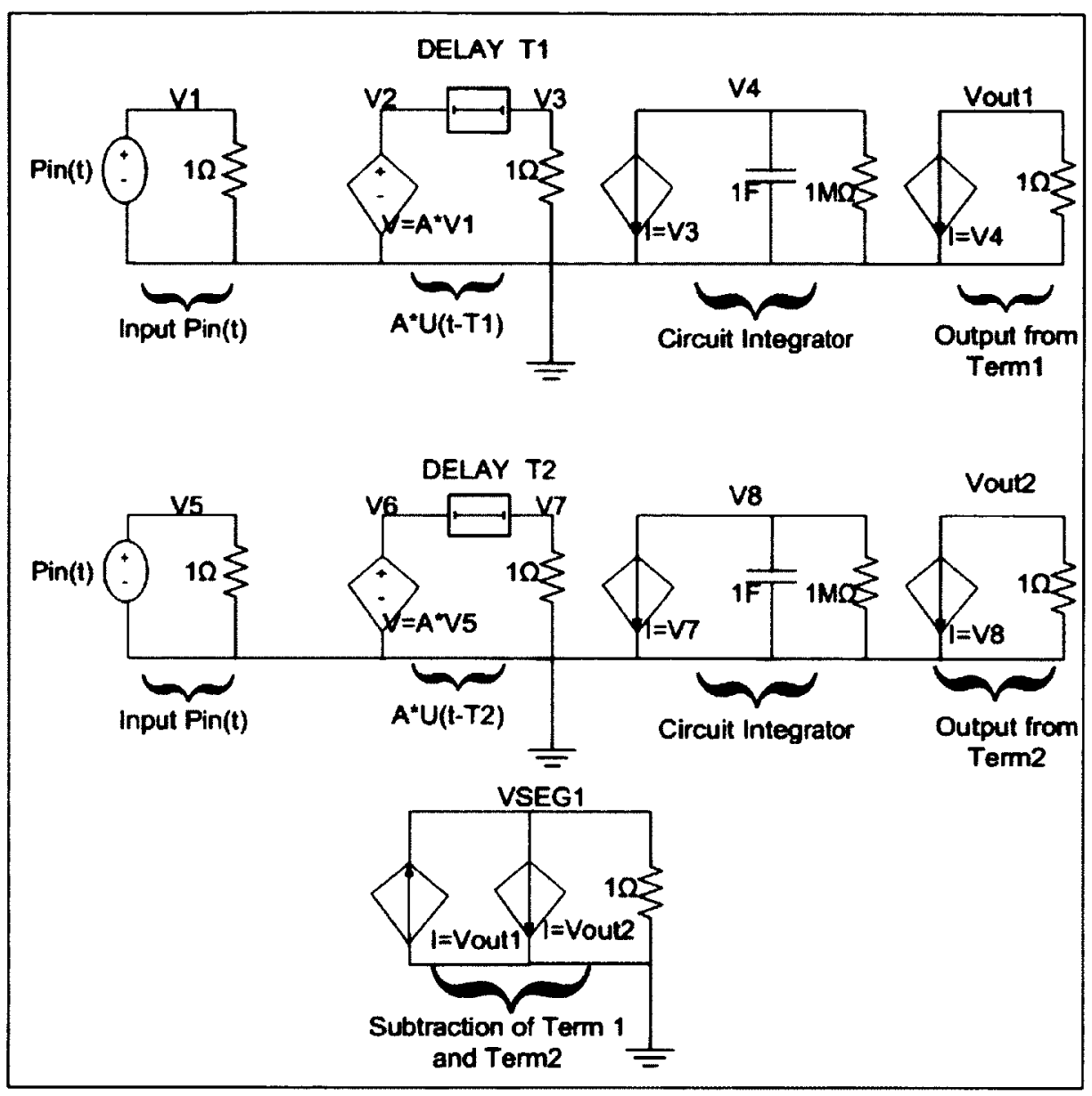

Figure 3.9: Sub-circuit of $N_{\text {seg1 }}(t)$ with $P_{\text {in }}(t)$ input

Note that Figure 3.9 has an input of $P_{i n}(t)$ and an output of voltage node VSEG1. This procedure is applied to every segment of $N_{\text {discrete }}(t)$ which creates $\mathrm{N}$ sub circuits as shown in Figure 3.10. 


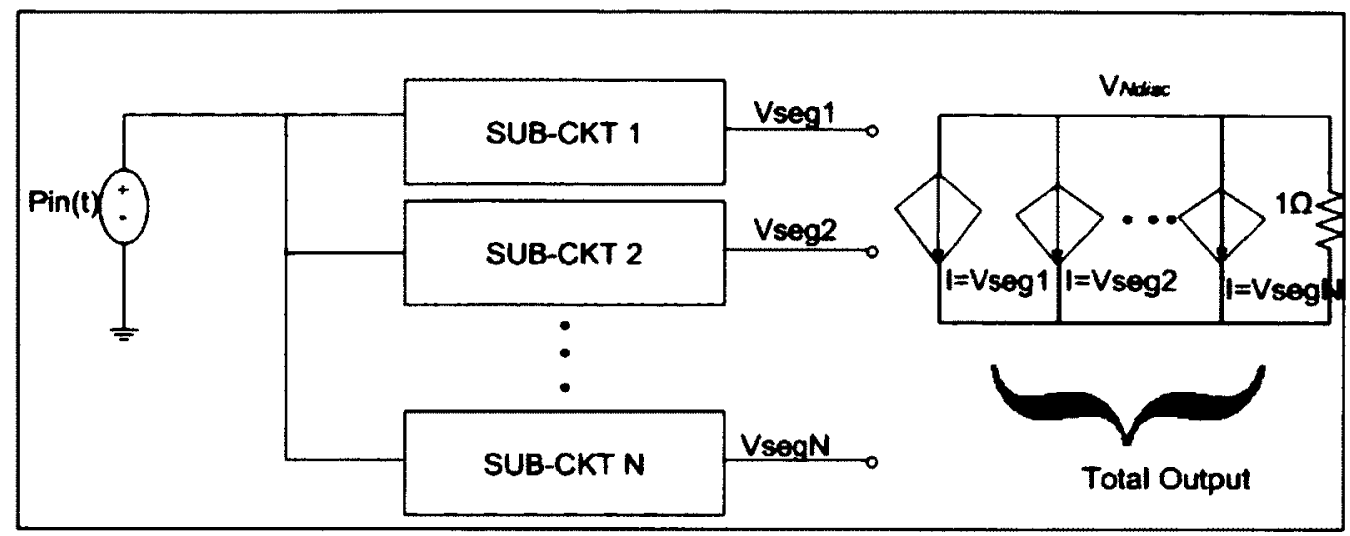

Figure 3.10: Circuit implementation of $N_{\text {discrete }}(t)$ with $P_{\text {in }}(t)$ input

In Figure 3.10, the input is $P_{i n}(t)$ and the output is the voltage of the node $V_{N d i s c}$. This voltage node can be used as a reference node for a voltage controlled current source that connects to the remaining parts of the functioning APD model.

Note that the signal from each segment will be added up with all the other segments. The superposition of each segment will contribute to the solution of this convolution operation. This circuit can be connected as a black box where the input is the same as the other subsystems and the output will give the current that will be added to the other subsystems. The $N(t)$ blocks of CE-APD, that contains the negative resistor, are replaced in QC-APD with an alternative stable circuit system that represents the $N(t)$ and thus mitigating the stability problem of CE-APD. The QCAPD replaced the $N(t)$ blocks that contained a negative resistor with an alternative stable circuit thus mitigating the stability issue.

The next step is to determine the QC-APD accuracy and simulation processing 
time as a function of the number of segments $\left(N_{\text {seg }}\right)$. Note that in this thesis, the simulation processing time refers to the total amount of time taken by the SPICE program to complete a circuit simulation, from start of program execution to end of program execution. The simulation time will refer to the stop time of the transient circuit analysis in SPICE.

\subsection{Effects of $N(t)$ Segmentation on Macromodel Performance}

A time domain simulation example is used to determine how the time domain response of the APD's simulation varies with respect to different number of segments $N_{\text {seg }}$. The example will consist of the APD from [2] where the parameters are given in Table 3.2. In order to compare accuracy, the FFT-APD macromodel will be used as a base for comparison to the QC-APD.

The example of the circuit for simulation is given in Figure 3.2 . 


\begin{tabular}{|c|c|}
\hline Parameter Names & Values \\
\hline \hline$P D_{e f f}$ & 0.5 \\
\hline$\alpha$ & $1.15 \mu \mathrm{m}^{-1}$ \\
\hline$W_{a}$ & $2.0 \mu \mathrm{m}$ \\
\hline $\mathrm{W}$ & $2.7 \mu \mathrm{m}$ \\
\hline$x_{m}$ & $0.5 \mu \mathrm{m}$ \\
\hline$x_{t}$ & $0.7 \mu \mathrm{m}$ \\
\hline$x_{a}$ & $2.7 \mu \mathrm{m}$ \\
\hline $1 / e_{h}$ & $4 \cdot 10^{-12} \mathrm{~s}$ \\
\hline $1 /\left(2 \pi \tau_{m}\right)$ & $60 \mathrm{GHz}$ \\
\hline$L$ & $0.3 \mathrm{nH}$ \\
\hline$C$ & $0.1 \mathrm{pF}$ \\
\hline$R$ & $60 \Omega$ \\
\hline$v_{p}$ & $4.8 \cdot 10^{6} \mathrm{~cm} / \mathrm{s}$ \\
\hline$v_{n}$ & $7 \cdot 10^{6} \mathrm{~cm} / \mathrm{s}$ \\
\hline$\alpha$ & $1.15 \mu \mathrm{m}^{-1}$ \\
\hline$M_{0}$ & 3 \\
\hline & \\
\hline
\end{tabular}

Table 3.2: Physical parameters of the InP/InGaAsP/InGaAs APD [2]

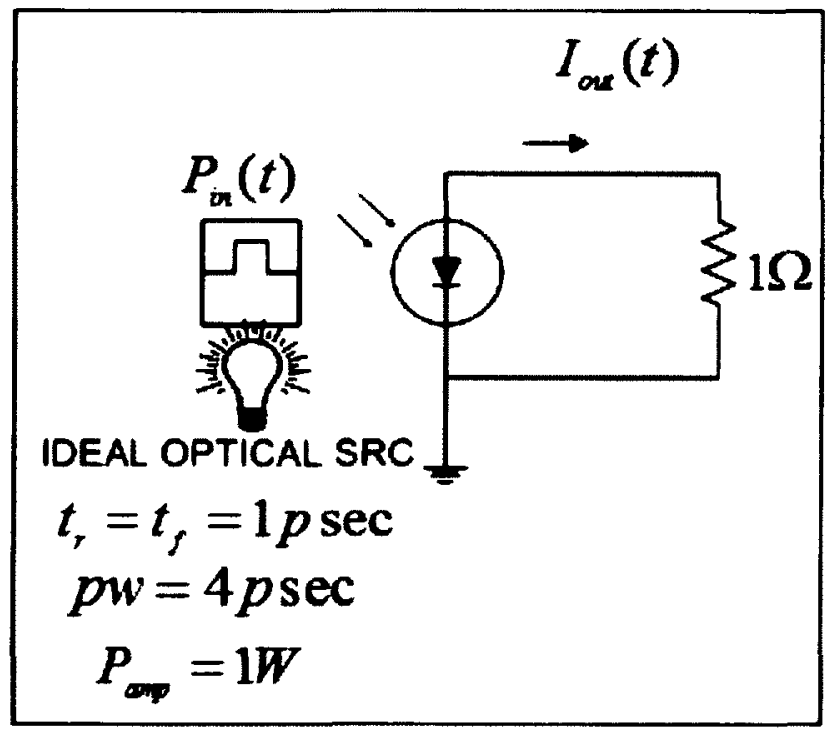

Figure 3.11: Circuit topology of APD with linear load

The computer hardware used to perform simulation processing time measurement 
is the following:

\begin{tabular}{|c|c|}
\hline Processor & $2.8 \mathrm{GHz}$ Intel Core 2 Duo \\
\hline Type & iMac 7.1 \\
\hline Number of processor & 1 \\
\hline Total number of core & 2 \\
\hline L2 Cache & $4 \mathrm{MB}$ \\
\hline Bus Speed & $800 \mathrm{MHz}$ \\
\hline Memory & $2 \mathrm{~GB} 667 \mathrm{MHz}$ DDR2 SDRAM \\
\hline
\end{tabular}

Table 3.3: Computer hardware used in simulation processing time measurements

\subsubsection{Simulation Processing Time and Accuracy Results for Different $N(t)$ Segments}

Figure 3.12 will shows the step response with different number of segments. Notice that as the number of segments increases from 2 to 1000 , the response appears to be matching with the response of the FFT-APD. Regardless of the number of segments, the QC APD response produces a stable response as shown in Figure 3.12. However, the simulation processing time increases with the increase in the number of segments as shown in Figure 3.13 . 


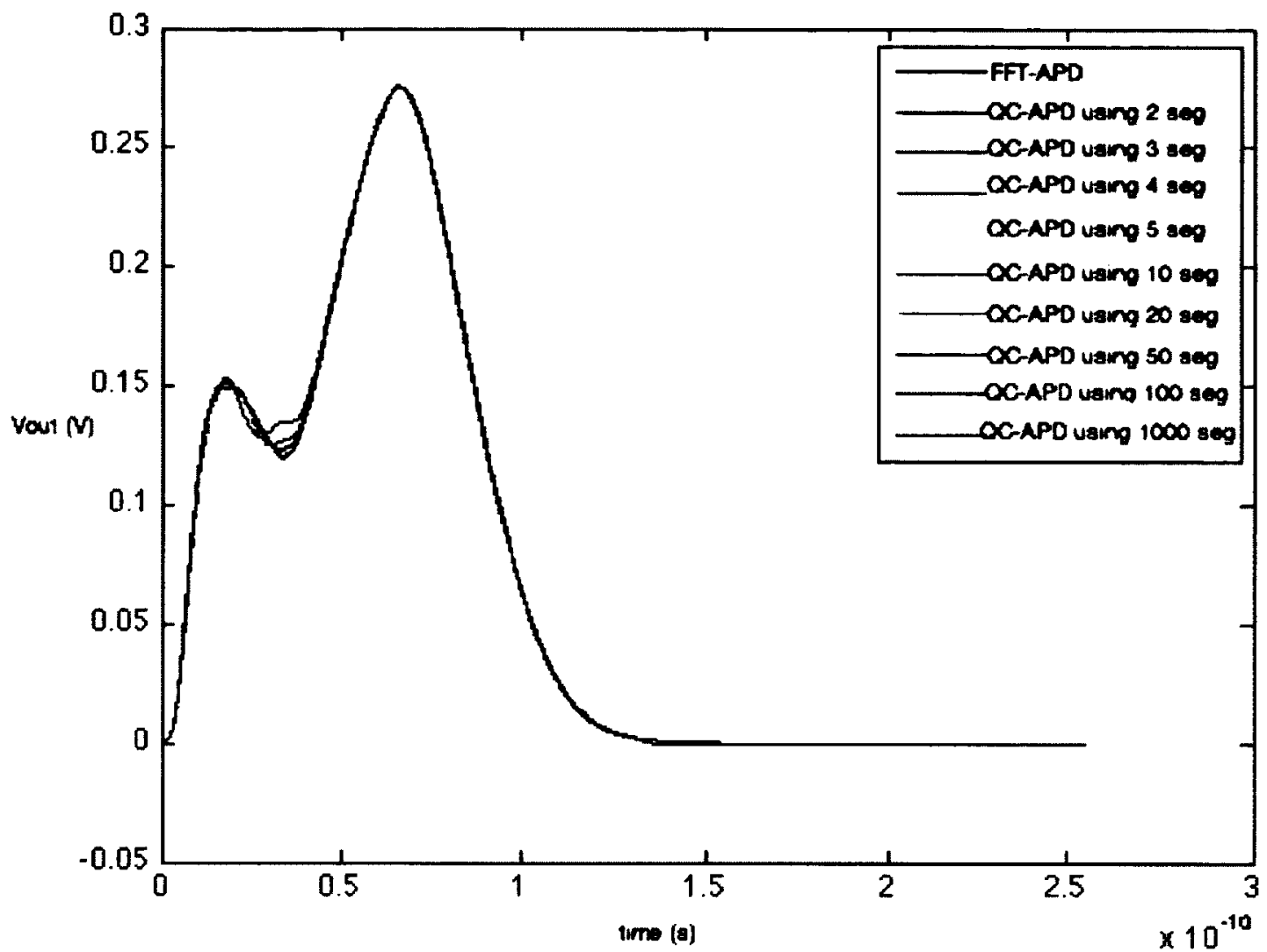

Figure 3.12: The APD step response with different number of segments $N$ seg

In this example, around 2 segments is enough to achieve the desired accuracy. At 2 segments, the simulation processing time was $0.42 \mathrm{sec}$. Further investigation will be performed in comparison to FFT-APD, Recursive Convolution APD macromodel and QC-APD macromodel in Chapter 5.

It can be concluded that for QC-APD, increasing the number of segments increases the simulation processing time and accuracy. Using the QC-APD formulations the unstable pole stays bounded and the simulation response stays stable. 


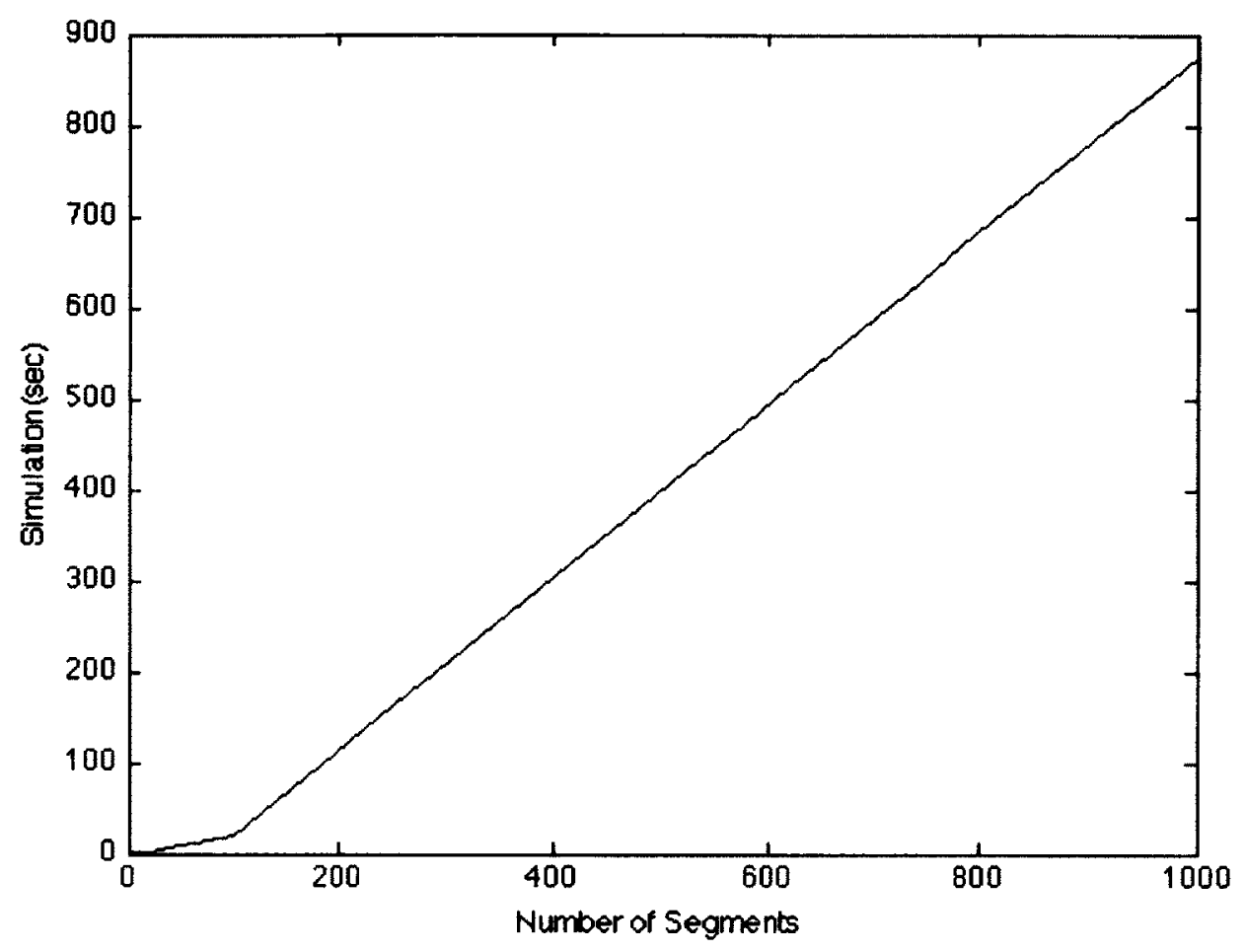

Figure 3.13: The simulation processing time of APD step response with different number of segments $N_{\text {seg }}$

The QC-APD model has the flexibility to be easily integrated into a SPICE-like engine because it is composed of circuit elements. It has the advantage of keeping most of the physical representation from the physical APD model. The next chapter will review the RC-APD macromodel and the effects of the number of fitted poles on simulation processing time and accuracy. 


\section{Chapter 4}

\section{Recursive Convolution APD}

\section{Macromodel}

This chapter will present a macromodel based on recursive convolution (RC-APD) and its accuracy and simulation processing time for different number of fitted poles. The first part of this chapter will present the RC-APD macromodel. The next section will present the accuracy and simulation processing time for different number of fitted poles. The final section will summarize the $\mathrm{RC}$ macromodel accuracy and simulation processing time. 


\subsection{Review of RC-APD macromodel}

In order to understand the RC-APD macromodel, the first subsection will review the recursive convolution technique [12-14]. The second subsection will review the frequency domain APD model [2]. The third subsection will review the vector fit algorithm (VF) [15-18] that will be used to approximate the frequency APD model.

\subsection{Review of RC Technique}

The origin of $\mathrm{RC}$ was in time domain simulation of transmission lines and digital interconnects [12-14]. The RC technique assumes that an admittance system is used in simulation, as shown in (4.1) [12].

$$
I(s)=Y(s) \cdot V(s)
$$

where $V(s)$ is the input voltage, $I(s)$ is the output current and $Y(s)$ is the admittance system. The admittance system must be in pole residue form as shown in (4.2) [12].

$$
Y(s)=\frac{k}{s-p}
$$


where $k$ is the residue and $p$ is the pole of the system. Using Laplace transform the impulse response of the system as shown in (4.3).

$$
\begin{aligned}
y(t) & =\mathfrak{£}^{-1}\{Y(s)\} \\
& =k e^{p t}
\end{aligned}
$$

The solution of admittance time domain impulse response solution is used in a time domain convolution integral as shown in (4.4)

$$
i(t)=\int_{0}^{t} k e^{p(t-\tau)} v(\tau) d \tau
$$

Thus the time domain integral convolution, using [12-14] is simplified into (4.5)

$$
i\left(t_{n}\right)=e^{p \cdot \Delta t} i\left(t_{n-1}\right)+v\left(t_{n}\right) \cdot \frac{k\left(1-e^{p \Delta t}\right)}{p}
$$

The simplified solution can be easily integrated into a simulation by representing into a constant current source and a conductance $[12-14]$ as shown in (4.6)

$$
i\left(t_{n}\right)=I_{e q}\left(t_{k}\right)+v\left(t_{k}\right) G_{e q}
$$


where:

$$
\begin{aligned}
G_{e q} & =\frac{k\left(1-e^{p \Delta t}\right)}{p} \\
I_{e q} & =e^{p \cdot \Delta t} i\left(t_{n-1}\right)
\end{aligned}
$$

Note that the $G_{e q}$ is known, the previous current is known, thus it can be stamped into Modal Node Analysis (MNA) and simulated as a circuit. The formulation for real, complex poles and multi-port examples can be shown in [12-14]. The next subsection will review frequency APD model such that it can be implemented using the $\mathrm{RC}$ technique.

\subsection{Review of InP, InGaAsP and InGaAs APD}

The frequency APD model [2] is given in (4.9).

$\frac{i_{s}(\omega)}{i_{s}(0)}=\frac{1}{M_{0}\left(1-e^{-\alpha W_{a}}\right)} \frac{1}{1-\omega^{2} L C+(j \omega R C)}\left[T_{n}(\omega)+T_{p}(\omega)+\frac{T_{n s}(\omega)}{\left[1+j \omega\left(M_{0}-1\right) t_{m}\right]\left(1+\frac{j \omega}{e_{h}}\right)}\right]$

where:

$$
T_{n}(\omega)=\frac{v_{n}}{W}\left[\left[\frac{1-e^{j \omega \frac{W_{a}}{v_{n}}}}{j \omega}\right]-e^{-\alpha W_{a}} \frac{\left[1-e^{-j \omega \frac{W_{a}}{v_{n}}+\alpha W_{a}}\right]}{j \omega-\alpha v_{n}}\right]
$$




$$
\begin{gathered}
T_{p}(\omega)=\frac{v_{p}}{W}\left[\frac{\left[1-e^{-\alpha W_{a}}\right]}{j \omega}\right. \\
\left.+\frac{\left[\frac{j \omega}{e_{h}}+e^{-\frac{j \omega x_{t}}{v_{p}}}\right]}{1+\frac{j \omega}{e_{h}}}\left[1-e^{\frac{-j \omega W_{a}}{v_{p}}-\alpha W_{a}}\right]\left[1-e^{\frac{-j \omega W_{a}}{v_{p}}-\alpha W_{a}}\right] \cdot\left[\frac{1}{j \omega+\alpha v_{p}}-\frac{1}{j \omega}\right]\right] \\
T_{n s}(\omega)=\frac{v_{n}}{W}\left(M_{0}-1\right)\left[e^{-\frac{j \omega x_{t}}{v_{p}}} \cdot\left[1-e^{-\frac{j \omega x_{a}}{v_{n}}}\right] \cdot\left[1-e^{\frac{-j \omega W_{\alpha}}{v_{p}}-\alpha W_{a}}\right] \cdot\left[\frac{1}{j \omega}-\frac{1}{\alpha v_{p}+j \omega}\right]\right]
\end{gathered}
$$

The problem with $(4.9)$ is that the term $T_{n}(\omega)$ has a time limited right hand pole. This time limited right hand pole can be modelled as a stable representation. This is done by approximating the frequency APD model with a rational function in pole residue form, using Vector Fit [15-17]. Therefore the next subsection will review the vector fit algorithm.

\subsection{Review of Vector Fit}

Vector Fit (VF) is a popular technique utilized to develop a model by fitting measured data or simulated data. The goal of VF is to find the accurate poles of a system that describes the given data [15-18]. The technique is developed by approximating the data by a rational function. The VF's resulting rational function is given in following form:

$$
f(s) \approx \sum_{n=1}^{N} \frac{k_{n}}{s-p_{n}}+c+s h
$$


where $\mathrm{f}(\mathrm{s})$ is the measured data, $k_{n}$ and $p_{n}$ are the $n^{\text {th }}$ pole and residue respectively, $\mathrm{N}$ is the number of poles and the number of residues, $\mathrm{c}$ and $\mathrm{h}$ are real constants. The challenge of $\mathrm{VF}$ is to estimate all the coefficients $\left(k_{n}, p_{n}, c, h\right)$ in the equation (4.13) such that the resulting rational function accurately approximates the measured or simulated data.

In order to approximate the simulated data with a pole residue function, a scaling function $\sigma(s)$ in (4.14) and $n$-pole user specified guess $\left(\bar{p}_{n}\right)$ are needed.

$$
\sigma(s)=\sum_{n=2}^{N} \frac{\tilde{k}_{n}}{s-\bar{p}_{n}}+1
$$

Next, Eqn. (4.14) can be multiplied by (4.13) and the result will yield to a scaled function, $F(s)$, given below.

$$
F(s)=\sigma(s) f(s)
$$

The solution to the scaled function $\sigma(s)$ can be approximated by (4.16) .

$$
\sigma(s) f(s) \approx \sum_{n=1}^{N} \frac{\hat{k}_{n}}{s-\bar{p}_{n}}+\hat{c}+\hat{h} s
$$

Note that $\hat{k}_{n}, \hat{c}$ and $\hat{h}$ are real constants that describe the approximated solution of the scaled function. 
Using (4.14) and (4.16) we have,

$$
\left[\sum_{n=1}^{N} \frac{\tilde{k}_{n}}{s-\bar{p}_{n}}+1\right] f(s) \approx\left[\sum_{n=1}^{N} \frac{\hat{k}_{n}}{s-\bar{p}_{n}}+\hat{c}+s \hat{h}\right]
$$

In (4.17), the $n$ poles represented by $\bar{p}_{i}$, where i $\epsilon 1 \ldots N$ are unknowns, and the total number of unknowns is given as $N_{\text {total }}=2 N+2$. Eqn. (4.17) can extend to different frequency points such that the number of frequency points should be greater than the number of unknowns. Thus making the system an overdetermined linear system. The overdetermined system is solved using [15-18] in order to get a new set of poles and residues. The new set of poles is fed back into the algorithm until the pole residue function converges to an effective approximation. The initial guess of poles affects the success of the pole residue approximation in matching the original response. The user has to determine the number of fitted poles as well as the initial pole guesses. In [15-17], it was noted that VF guarantees that the fitted poles are stable. The next section will describe the RC-APD macromodel development. 


\subsection{Development of RC based APD Time Domain Model}

The development of RC will combine all the reviewed concepts and apply to the development of the RC-APD. The procedure to develop an RC-APD macromodel is shown in Figure 4.1. The development includes the solution of frequency APD model up to maximum frequency $f_{\max }$ and frequency step size $\Delta f$. Note that $f_{\max }$ is decided using the rise-time of the input signal. The VF technique must be used to find the accurate approximation of a rational function to the simulated frequency response. The user has to find the number of fitted poles and their values in order to give VF a starting point. Then the user must verify that VF produces an accurate approximation of the frequency APD response. Once the verification is complete, the VF solved pole residue functions are placed into the $\mathrm{RC}$ formulation such that they can be solved in the circuit simulator. The next section will discuss the speed and accuracy of the RC-APD macromodel for different number of fitted poles. 


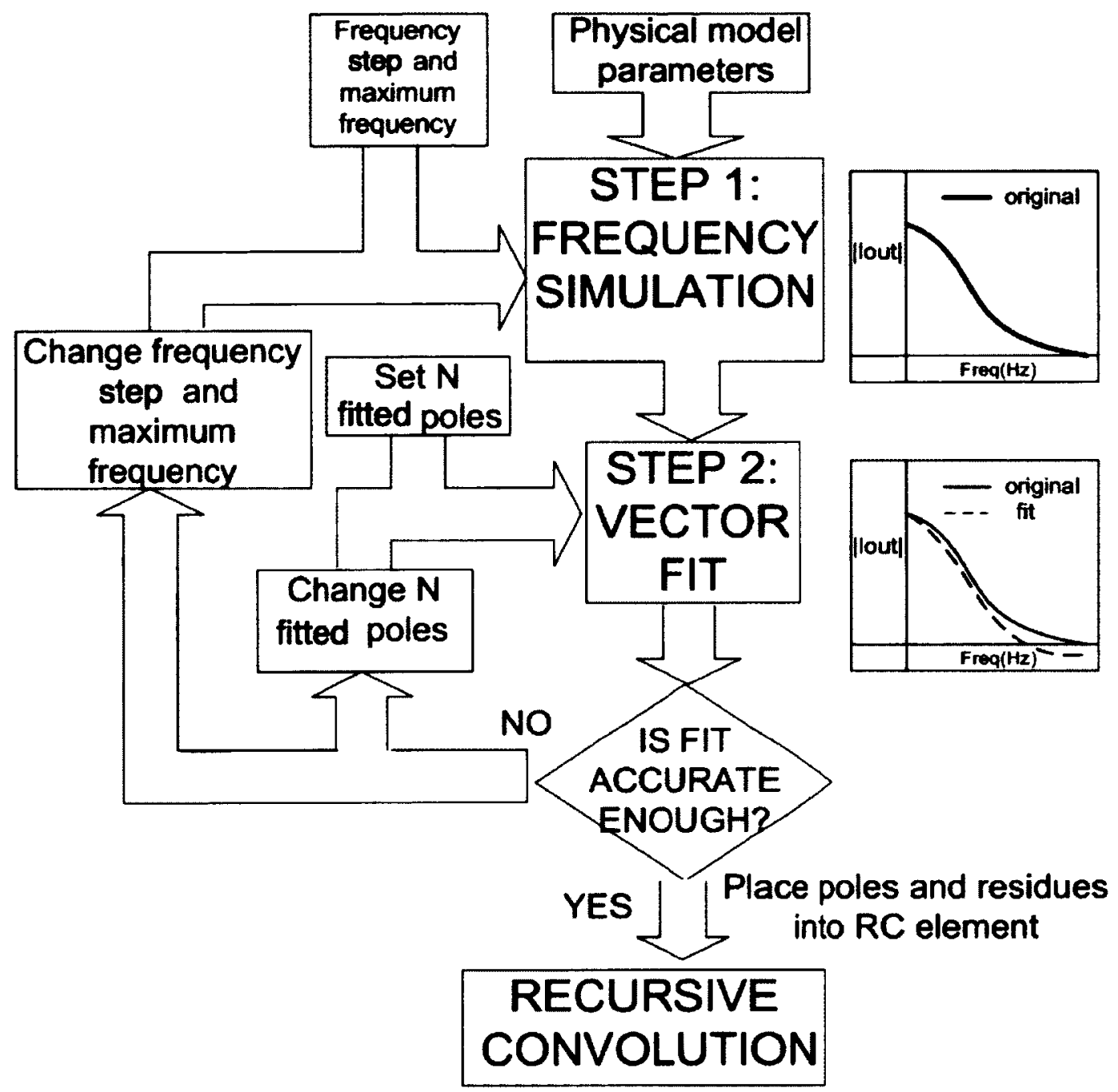

Figure 4.1: Development of RC-APD macromodel

Note that in Figure 4.1, $\mathrm{N}$ refers to the number of fitted poles. 


\subsection{Testing Accuracy and Simulation Processing Time of RC-APD Macromodel}

A circuit composed of an APD [2] with a load resistor is used to test the accuracy and simulation processing time of the RC-APD macromodel as shown in Figure 4.2.

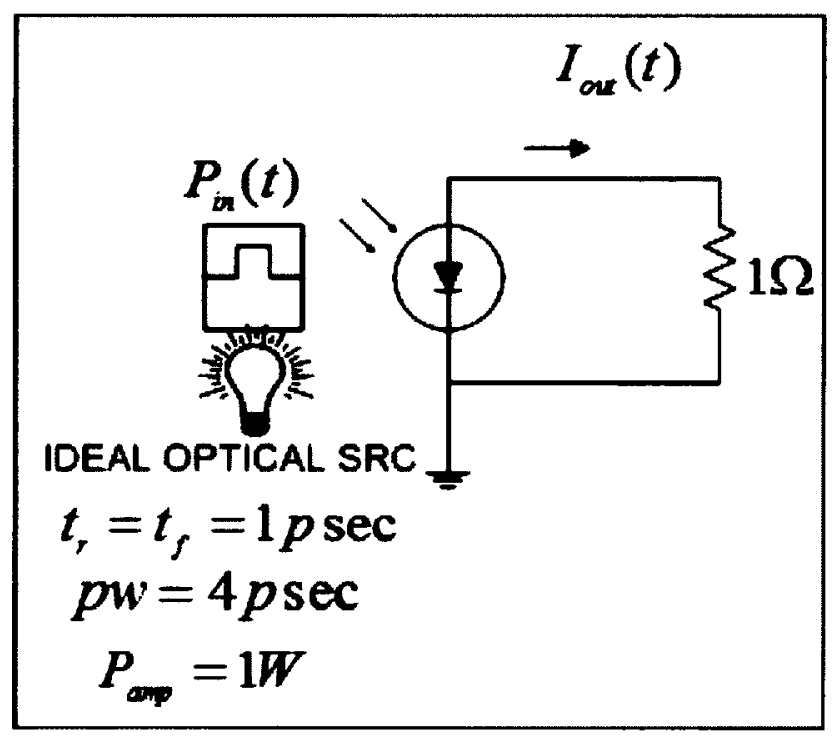

Figure 4.2: Circuit topology of RC-APD with resistive load

The APD model parameters are given in Table 4.2. The macromodel parameters are given in Table 4.3. The FFT-APD is used as a comparison to test the accuracy of the results. The computer hardware used to perform simulation processing time measurement is the following: 


\begin{tabular}{|c|c|}
\hline Processor & $2.8 \mathrm{GHz}$ Intel Core 2 Duo \\
\hline Type & iMac 7.1 \\
\hline Number of processor & 1 \\
\hline Total number of core & 2 \\
\hline L2 Cache & $4 \mathrm{MB}$ \\
\hline Bus Speed & $800 \mathrm{MHz}$ \\
\hline Memory & $2 \mathrm{~GB} 667 \mathrm{MHz}$ DDR2 SDRAM \\
\hline
\end{tabular}

Table 4.1: Computer hardware used in simulation processing time measurements

\begin{tabular}{|c|c|}
\hline Parameter Names & Values \\
\hline$P D_{\text {eff }}$ & 0.5 \\
\hline$\alpha$ & $1.15 \mathrm{um}^{-1}$ \\
\hline$W_{a}$ & $2.0 \mu \mathrm{m}$ \\
\hline $\mathrm{W}$ & $2.7 \mu \mathrm{m}$ \\
\hline$x_{m}$ & $0.5 \mu \mathrm{m}$ \\
\hline$x_{t}$ & $0.7 \mu \mathrm{m}$ \\
\hline$x_{a}$ & $2.7 \mu \mathrm{m}$ \\
\hline$M_{0}$ & 10 \\
\hline $1 / e_{h}$ & $4 \cdot 10^{-12} \mathrm{~s}$ \\
\hline $1 /\left(2 \pi \tau_{m}\right)$ & $60 \mathrm{GHz}$ \\
\hline$L$ & $0.3 \mathrm{nH}$ \\
\hline$C$ & $0.1 \mathrm{pF}$ \\
\hline$R$ & $60 \Omega$ \\
\hline$v_{p}$ & $4.8 \cdot 10^{6} \mathrm{~cm} / \mathrm{s}$ \\
\hline$v_{n}$ & $7 \cdot 10^{6} \mathrm{~cm} / \mathrm{s}$ \\
\hline$\alpha$ & $1.15 \mu \mathrm{m}^{-1}$ \\
\hline
\end{tabular}

Table 4.2: Physical parameters of the InP/InGaAsP/InGaAs APD

\begin{tabular}{|c|c|}
\hline Parameter Name & Value \\
\hline Number of Poles & $2,16,48$ \\
\hline Range of Initial Poles (logarithmically spaced) & $10^{7}-10^{12} \mathrm{~Hz}$ \\
\hline Frequency Range & $0-300 \mathrm{GHz}$ \\
\hline Fstep & $30.003 \mathrm{MHz}$ \\
\hline
\end{tabular}

Table 4.3: Example 1:RC based APD model parameters 


\subsubsection{Simulation Results of Accuracy Control}

The time domain circuit response for different number of fitted poles is shown in Figures 4.3 and 4.4. In Figure 4.3, with 2 and 4 fitted poles, the APD response only captures part of the response because the VF can only accurately fit certain parts of the whole frequency response. As the number of initial poles is increased to $16, \mathrm{VF}$ can fit the frequency response with greater accuracy and across a larger frequency range and thus the time domain response matches the response of the APD-FFT.

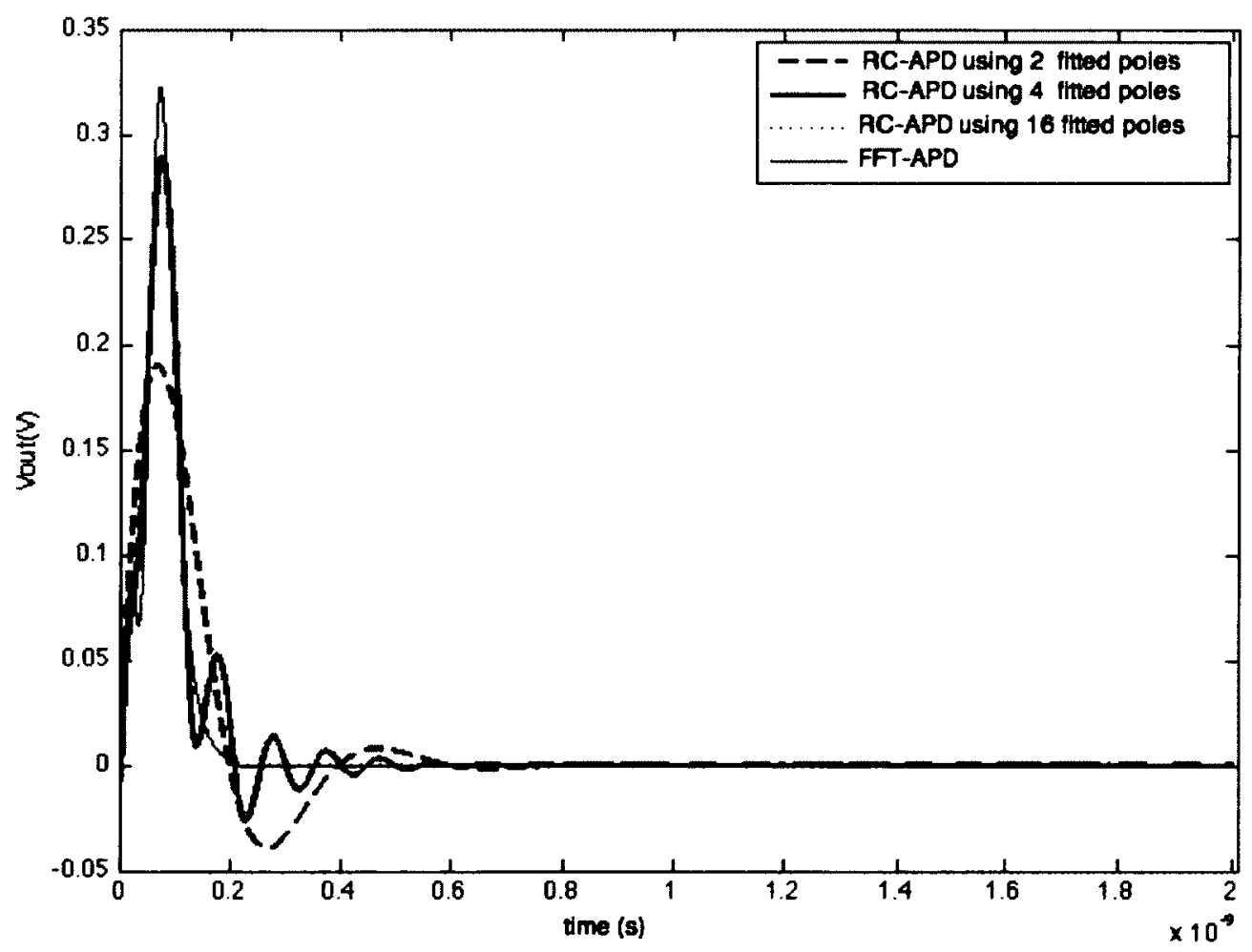

Figure 4.3: Effects of number of pole changes on accuracy of time domain response using RC-APD macromodel as compared to FFT-APD 
When the number of fitted poles is increased from 16 to 32 , the extra poles introduced greater RMS (Root Mean Square) error from $419.4 \cdot 10^{-6}$ to $605.3 \cdot 10^{-6}$ $(\sqrt{A})$ and thus the time response becomes inaccurate as shown in Figure 4.4. If the number of fitted poles is chosen to be 48 , the time domain response is inaccurate. VF algorithm's internal matrix solution develops a rank problem because the resulting model faces passivity problems.

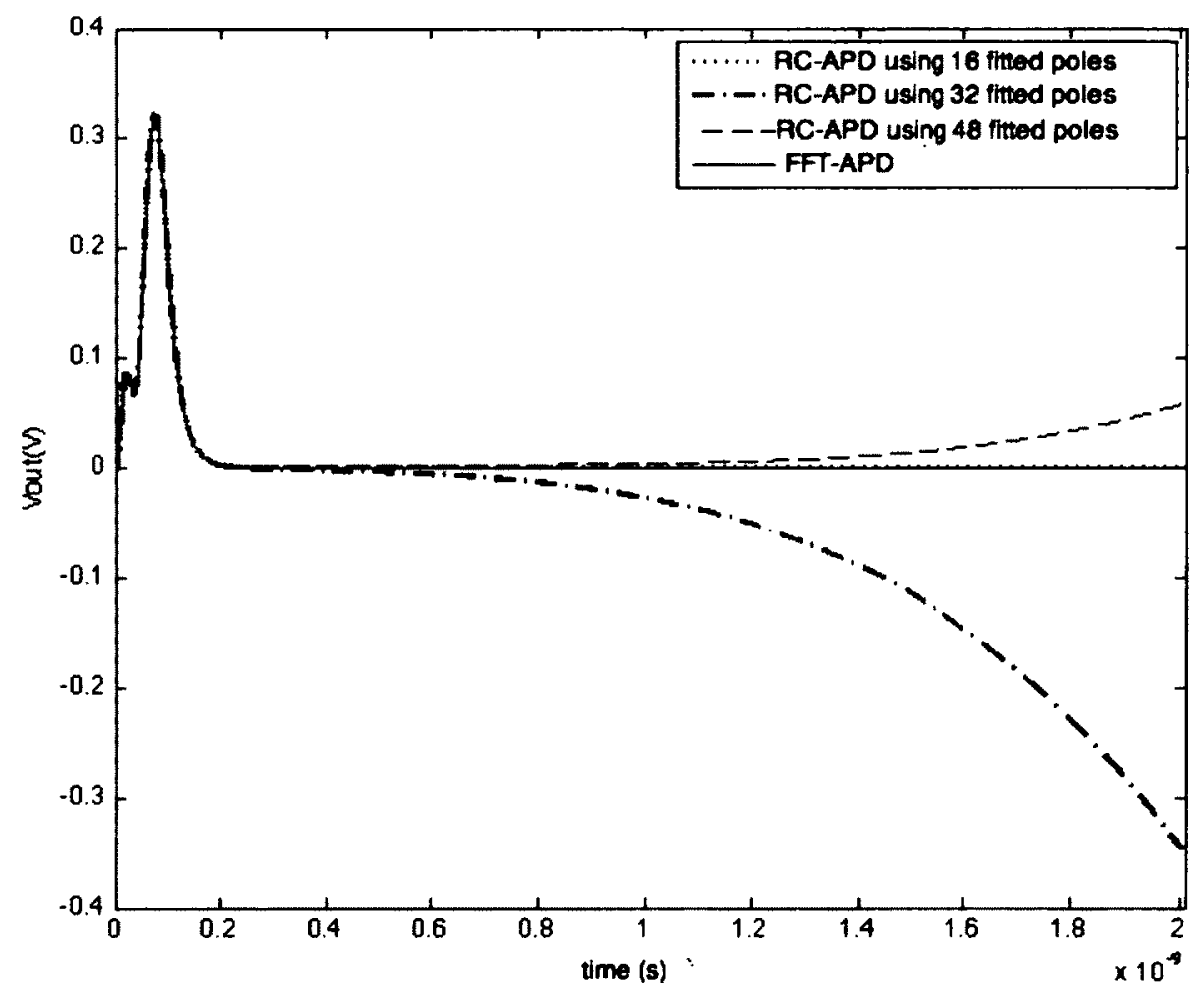

Figure 4.4: Effects of number of pole changes on accuracy of time domain response using RC based APD macromodel as compared to FFT-APD

The next section will review the change in simulation speed as the number of 
fitted poles increase.

\subsubsection{Simulation Results of Simulation Processing Time}

In Figure 4.4, it was shown that when we used more than 16 fitted poles, the APD response produced inaccurate results. Coincidently, the simulation processing time spiked when more than 16 fitted poles was used to generate the APD model, shown in Figure 4.5. These inaccurate results and the spike in simulation processing time are mainly due to a rank deficiency problem in the models generated by Vector Fit. Nevertheless, this model problem causes simulator convergence issues that contribute to the increase in simulation processing time. 


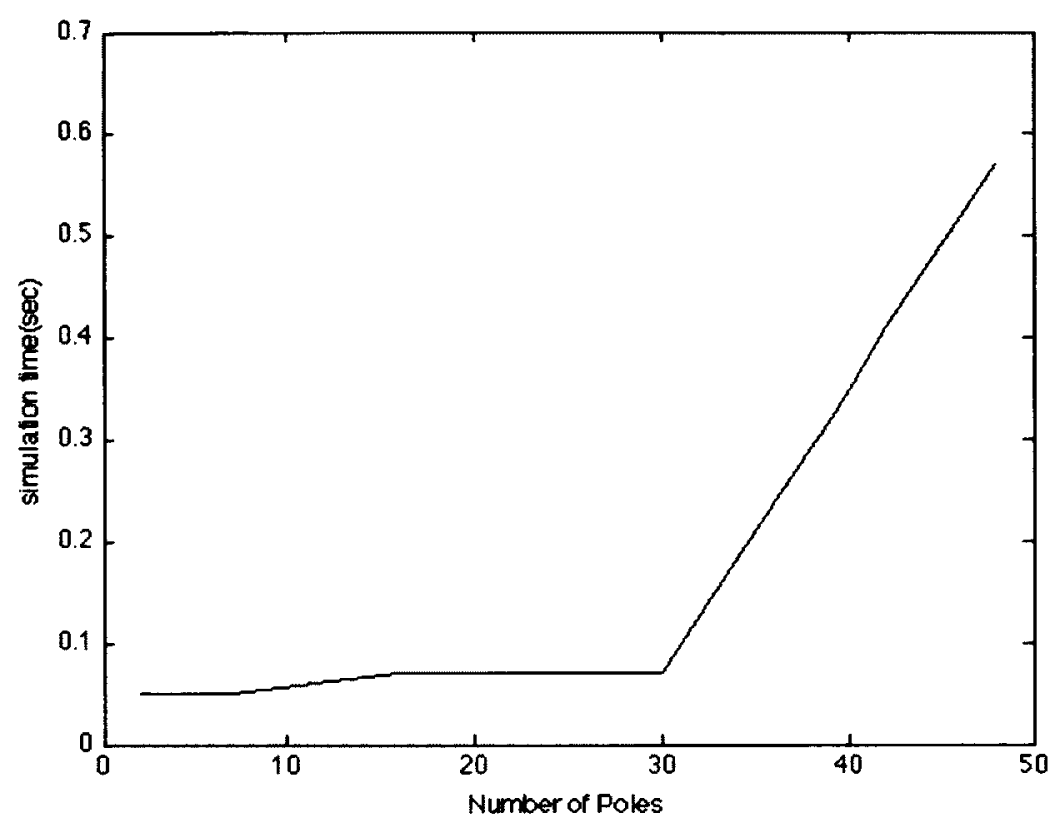

Figure 4.5: Number of fitted poles vs simulation processing time of RC based APD macromodel

\subsection{Summary of Results}

For the RC-APD macromodel, the user has to determine the appropriate number of fitted poles and their values for the starting point of VF. If a certain number of fitted poles is used, VF can face rank deficiency problems in it solution. This happens because the resulting model is facing passivity problems.

In the next chapter the FFT-APD, QC-APD and RC-APD are compared for speed, accuracy and implementation issues. 


\section{Chapter 5}

\section{Macromodel Comparison for}

\section{Simulation Processing Time,}

\section{Accuracy and Macromodel}

\section{Implementation Issues}

Chapters 3 and 4 presented the QC-APD and RC-APD and their accuracy and simulation processing time. In this chapter, the RC-APD and QC-APD are compared for simulation processing time, accuracy and macromodel implementation issues. The FFT-APD is used as a reference for the other macromodels.

The first section will review the methods of comparison for simulation process- 
ing and accuracy. The second section will present simulation processing time and accuracy comparison of APD macromodels for a circuit with a linear termination. The third section will present simulation processing time and accuracy comparison of APD macromodels for a circuit with a nonlinear termination. The third section will compare implementation issues between the RC-APD and the QC-APD. The implementation issues are illustrated by presenting results of multiple time domain simulation examples where the APD model parameters $\alpha$ and $v_{n}$ are varied, the nonlinear termination is varied and the input trapezoidal pulse is varied.

\subsection{Methods of Comparison}

The comparison of FFT, QC and RC based APD macromodels must be done on identical circuits and it must consider the effects of variable time step methods. The variable time step methods are internal SPICE algorithms [4] that attempt to reduce overall simulation processing time and improve accuracy by varying the time step at different instances of the simulation.

The first part of macromodel comparison will be performed on examples where the variable time step methods are disabled by forcing a fixed time step. A second part of the macromodel comparison will be run with variable time stepping enabled. Note that the simulation speed refers to the speedup of the simulation processing time. 


\subsubsection{Fixed Time Stepping}

In this subsection, the analysis of the models with respect to accuracy and simulation speed are presented. The analysis will be structured in the following way:

\section{Accuracy}

The accuracy comparison cases are the following:

1. Compare the time domain responses of each macromodel for a given example.

2. Compare the relative accuracy change between the two macromodels when their internal parameters are changed.

3. Compare the time domain responses of each macromodel for different fixed time steps.

\section{Simulation Speed}

The simulation processing speed comparison cases are the following:

1. Compare the simulation processing time versus the number of time points for the given example.

2. Compare the simulation processing time by finding the relative speedup. 
The speedup is calculated as:

$$
\text { Speedup }=\frac{T_{\text {simMethod } 1}}{T_{\text {simMethod } 2}}
$$

where:

Speedup - is the ratio of simulation processing time of method 1 with respect to method 2

$T_{\text {simMethod } 1}$ - is the time simulation processing time of method 1 $T_{s i m M e t h o d 2}-$ is the time simulation processing time of method 2

The first example will be based on an APD circuit with a linear resistive capacitive termination. The second example will be a simulation of a transimpedance amplifier (TIA), a common nonlinear termination in optical communication [19]. Refer to Appendix A for more details on the TIA. The third example will repeat example 2 for different input signal rise time, $\alpha, v_{n}$ and different nonlinear loads. Note the example 1,2 and 3 will be compared using cases 1 and 2 of accuracy and simulation speed comparison for fixed time stepping.

\subsubsection{Variable Time Stepping}

In this subsection, the accuracy and simulation speed cases are presented. 


\section{Accuracy}

The accuracy comparison case is the following:

1. Compare the time domain responses of each macromodel for a given example.

\section{Simulation Speed}

The simulation speed comparison case is the following:

1. Compare the number of time points of each macromodel for different input signal rise time.

The fourth example will be an APD with a linear load where case 3 of the accuracy comparison is performed. The fifth example will be an APD with a linear load using the comparison methods for variable time step. These examples can illustrate the implementation issues with variable and fixed step sizing.

\subsection{Time Domain Simulation of APD with a Lin- ear Load}

In this section, the accuracy and simulation processing time of the macromodels is tested on two examples: The first example is an APD [2] that is connected to a 
resistive/capacitive termination as shown in Figure 5.1. The APD model parameters, from the APD shown in Figure 5.1, are given in Table 5.1.

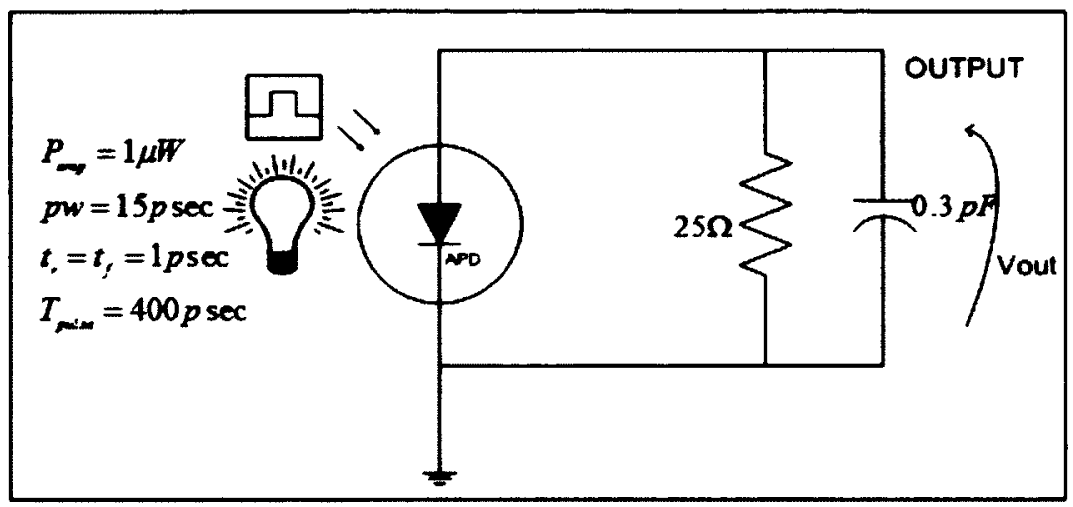

Figure 5.1: Circuit of APD terminated with a linear load

\begin{tabular}{|c|c|}
\hline Parameter Names & Values \\
\hline \hline$P D_{\text {eff }}$ & 0.5 \\
\hline$\alpha$ & $1.15 \mu \mathrm{m}^{-1}$ \\
\hline$W_{a}$ & $2.0 \mu \mathrm{m}$ \\
\hline $\mathrm{W}$ & $2.7 \mu \mathrm{m}$ \\
\hline$x_{m}$ & $0.5 \mu \mathrm{m}$ \\
\hline$x_{t}$ & $0.7 \mu \mathrm{m}$ \\
\hline$x_{a}$ & $2.7 \mu \mathrm{m}$ \\
\hline$M_{0}$ & 2 \\
\hline $1 / e_{h}$ & $4 \cdot 10^{-12} \mathrm{~s}$ \\
\hline $1 /\left(2 \pi \tau_{m}\right)$ & $60 \mathrm{GHz}$ \\
\hline$L$ & $0.3 \mathrm{nH}$ \\
\hline$C$ & $0.1 \mathrm{pF}$ \\
\hline$R$ & $60 \Omega$ \\
\hline$v_{p}$ & $4.8 \cdot 10^{6} \mathrm{~cm} / \mathrm{s}$ \\
\hline$v_{n}$ & $7 \cdot 10^{6} \mathrm{~cm} / \mathrm{s}$ \\
\hline$\alpha$ & $1.15 \mu \mathrm{m}^{-1}$ \\
\hline$M_{0}$ & 2 \\
\hline
\end{tabular}

Table 5.1: Physical parameters of the InP/InGaAsP/InGaAs APD [2]

The macromodel parameters used in this simulation example are shown in Tables 
$5.2,5.2$ and 5.4 .

\begin{tabular}{|c|c|}
\hline Parameter Name & Value \\
\hline Number of Segments & 20 \\
\hline
\end{tabular}

Table 5.2: QC-APD macromodel parameters

\begin{tabular}{|c|c|}
\hline Parameter Name & Value \\
\hline Frequency Range & $0-150 \mathrm{GHz}$ \\
\hline Fstep & $4.514 \mathrm{MHz}$ \\
\hline
\end{tabular}

Table 5.3: FFT-APD macromodel parameters

\begin{tabular}{|c|c|}
\hline Parameter Name & Value \\
\hline Number of fitted Poles & 16 \\
\hline Range of Poles Values(logarithmically spaced) & $10^{7}-10^{12} \mathrm{~Hz}$ \\
\hline Frequency Range & $0-170 \mathrm{GHz}$ \\
\hline Fstep & $4.51 \mathrm{MHz}$ \\
\hline
\end{tabular}

Table 5.4: RC-APD macromodel parameters

The computer hardware used to perform simulation processing time measurement is the following:

\begin{tabular}{|c|c|}
\hline Processor & $2.8 \mathrm{GHz}$ Intel Core 2 Duo \\
\hline Type & iMac 7.1 \\
\hline Number of processor & 1 \\
\hline Total number of core & 2 \\
\hline L2 Cache & $4 \mathrm{MB}$ \\
\hline Bus Speed & $800 \mathrm{MHz}$ \\
\hline Memory & $2 \mathrm{~GB} 667 \mathrm{MHz}$ DDR2 SDRAM \\
\hline
\end{tabular}

Table 5.5: Computer hardware used in simulation processing time measurements 


\subsubsection{Simulation Processing Time Results}

The simulation processing time versus the number of total simulation points is shown in Figure 5.2. As the total number of simulation points increase the simulation processing time increases linearly for each of the APD models. At 2500000 time points, FFT simulation processing is just under 1400 seconds while QC-APD requires about 200 second and RC-APD requires about 20 seconds to complete the simulation time.

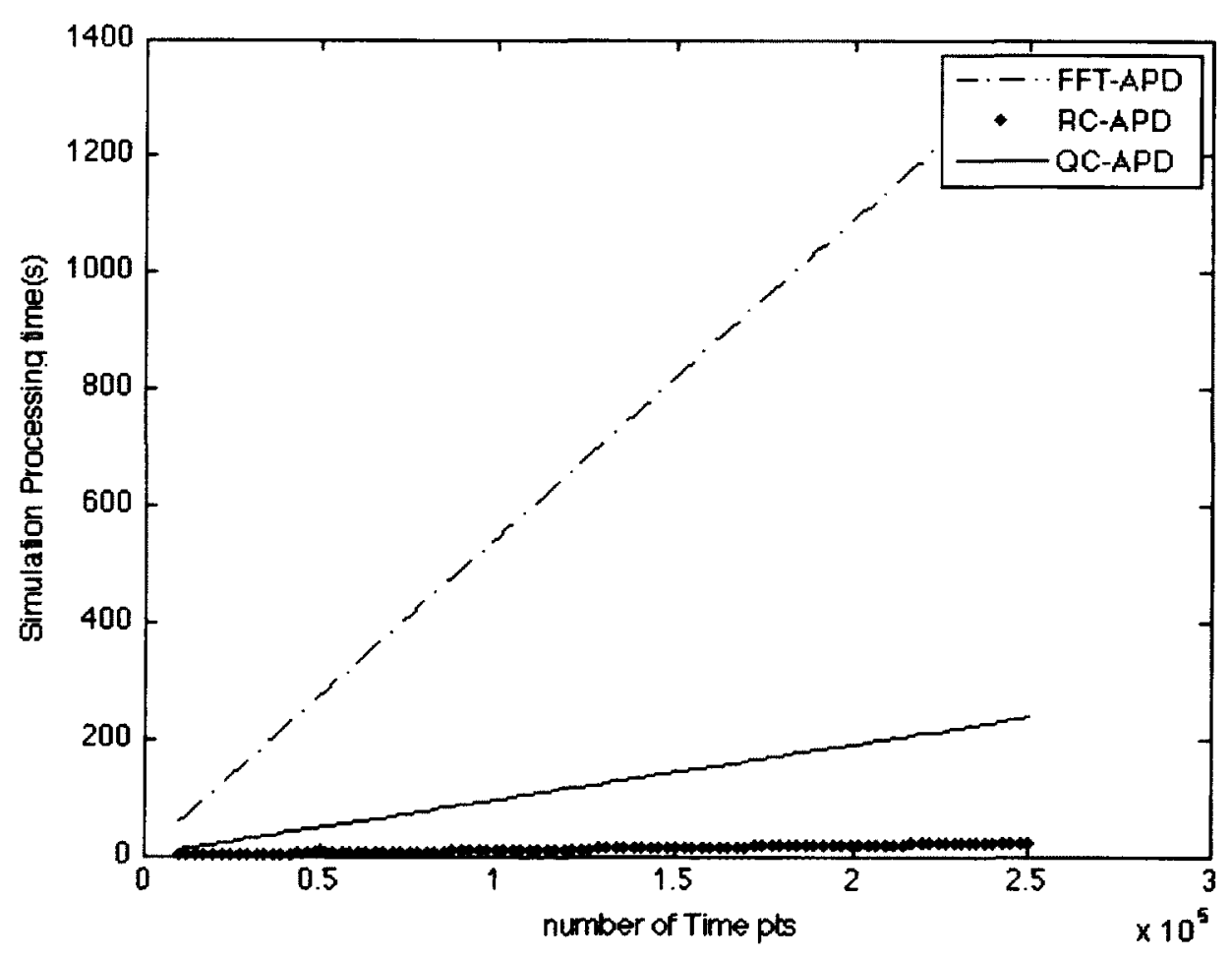

Figure 5.2: Simulation processing time (sec) versus the number of simulation time points for FFT, QC-APD and RC based APD 
In Figure 5.2, RC-APD has the smallest simulation processing time, followed by QC-APD and the longest simulation processing time is taken by the method FFT. In order to summarize the simulation processing time comparison Table 5.6 will present the average speedup for this example results between each of the three methods.

\begin{tabular}{|c|c|c|c|}
\hline Method 1 & Method 1:RC-APD & Method 1:QC-APD & Method 1: FFT-APD \\
\hline FFT-APD & 57 & 5.7 & 1.0 \\
\hline QC-APD & 10 & 1.0 & $<1$ \\
\hline RC-APD & 1.0 & $<1$ & $<1$ \\
\hline
\end{tabular}

Table 5.6: Macromodel speedup comparison

The speedup of RC to FFT is 57 and 10 as compared to QC-APD for this example. The speedup of each of the model is consistent for this example across any number of simulation time points.

It can be concluded that RC-APD is the fastest macromodel, followed closely by QC-APD and FFT-APD which is very slow. The next comparison will compare the macromodel accuracy.

\subsubsection{Accuracy Results of the Three Macromodels}

The time domain responses of FFT-APD, QC-APD and RC-APD macromodel are shown in Figure 5.3. The three macromodels give an identical response as shown in Figure 5.3. 


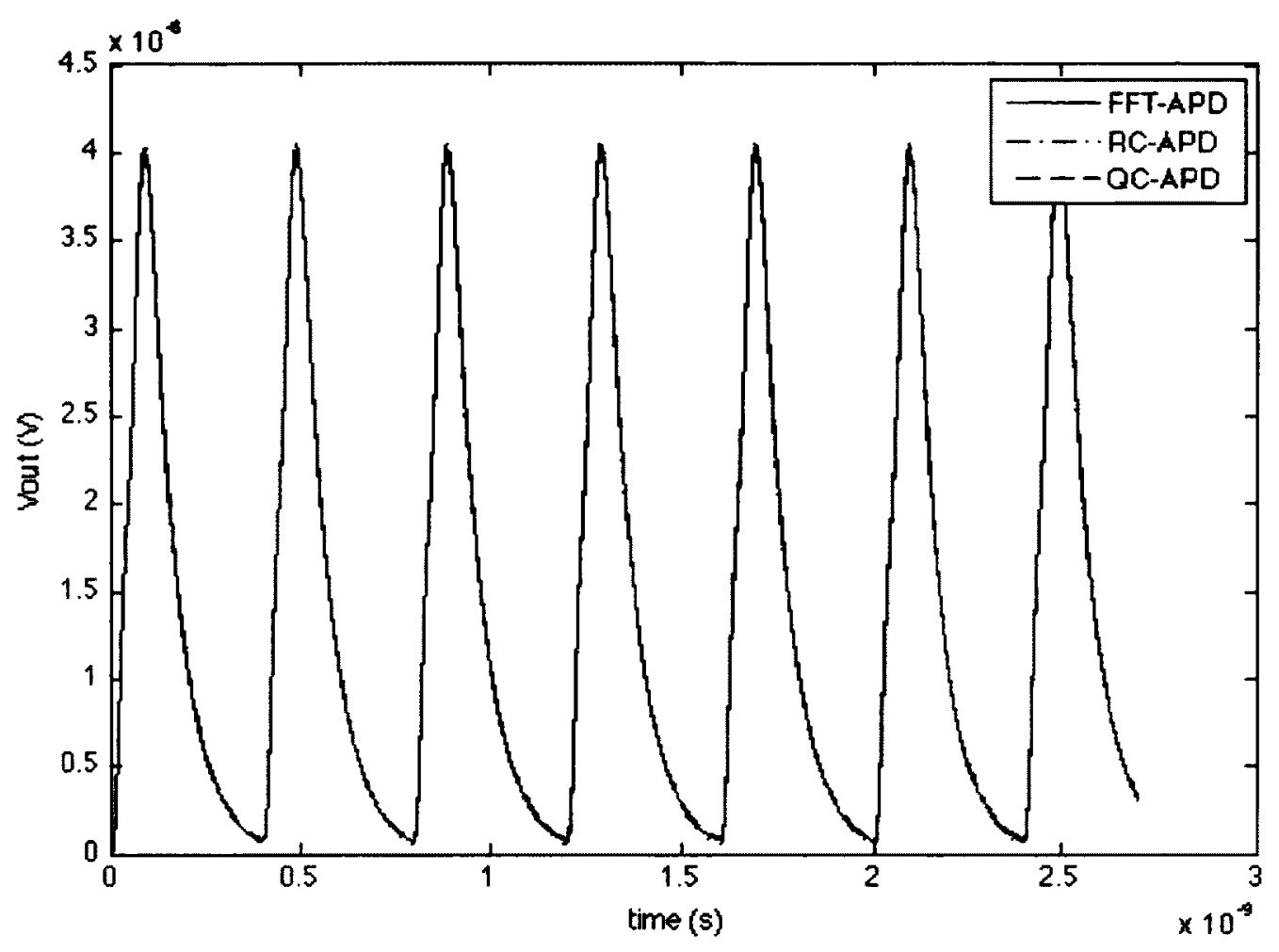

Figure 5.3: Time Domain Response of Circuit from Figure 5.1

If the number of fit poles and number of segments are changed, the change is reflected in the circuit response as shown in Figure 5.4, 5.5, 5.6, 5.7 and Figure 5.8. Once again, increasing the number of segments improves accuracy of the QC-APD macromodel. In the case of RC-APD, overly increasing the number of fitted poles creates an inaccurate circuit response as shown in Figure 5.8. 


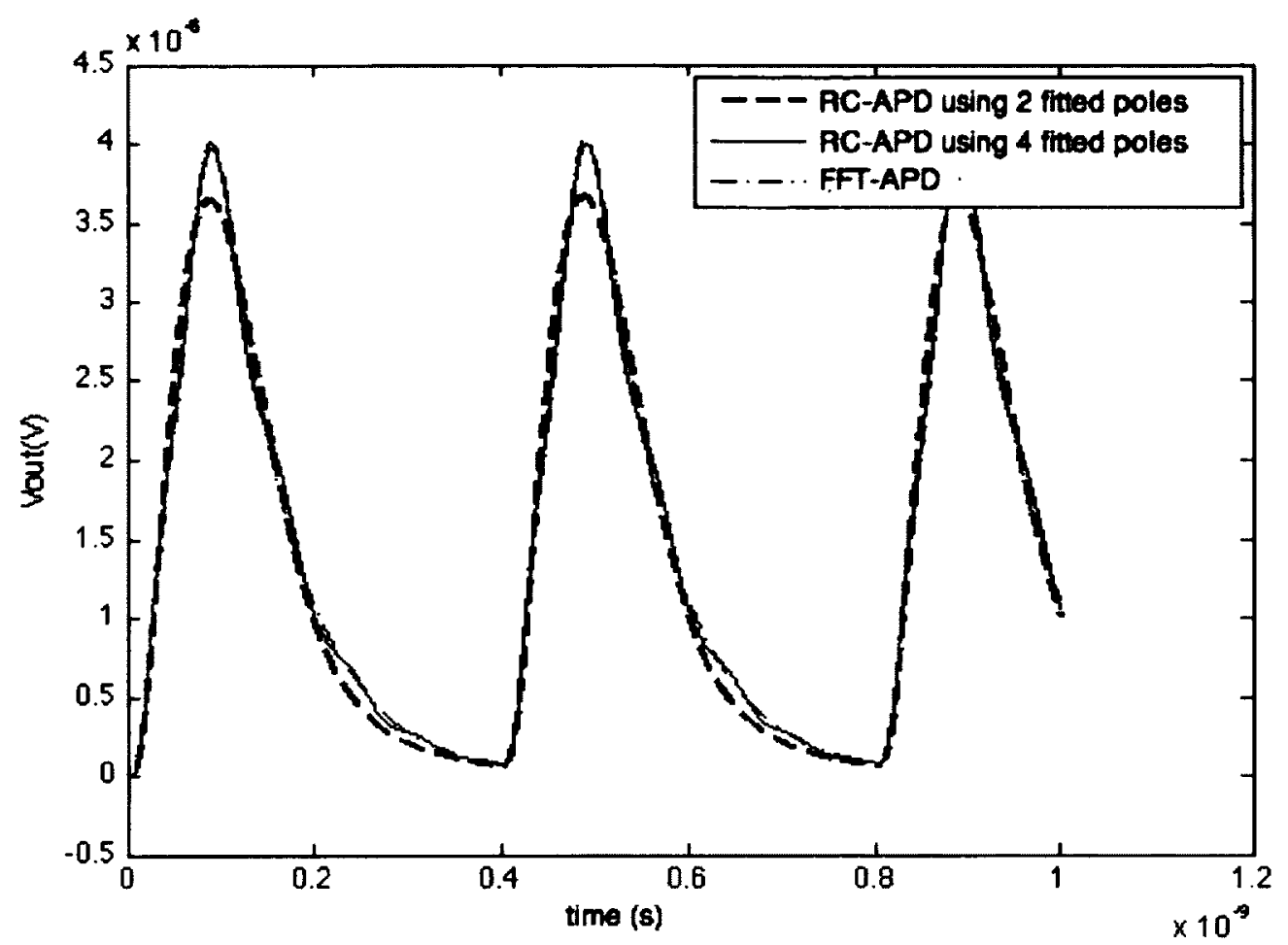

Figure 5.4: RC-APD response for different number of fitted poles

The minimum number of fitted poles required to VF the time domain response for RC-APD macromodel are 4 poles as shown in Figure 5.4. 


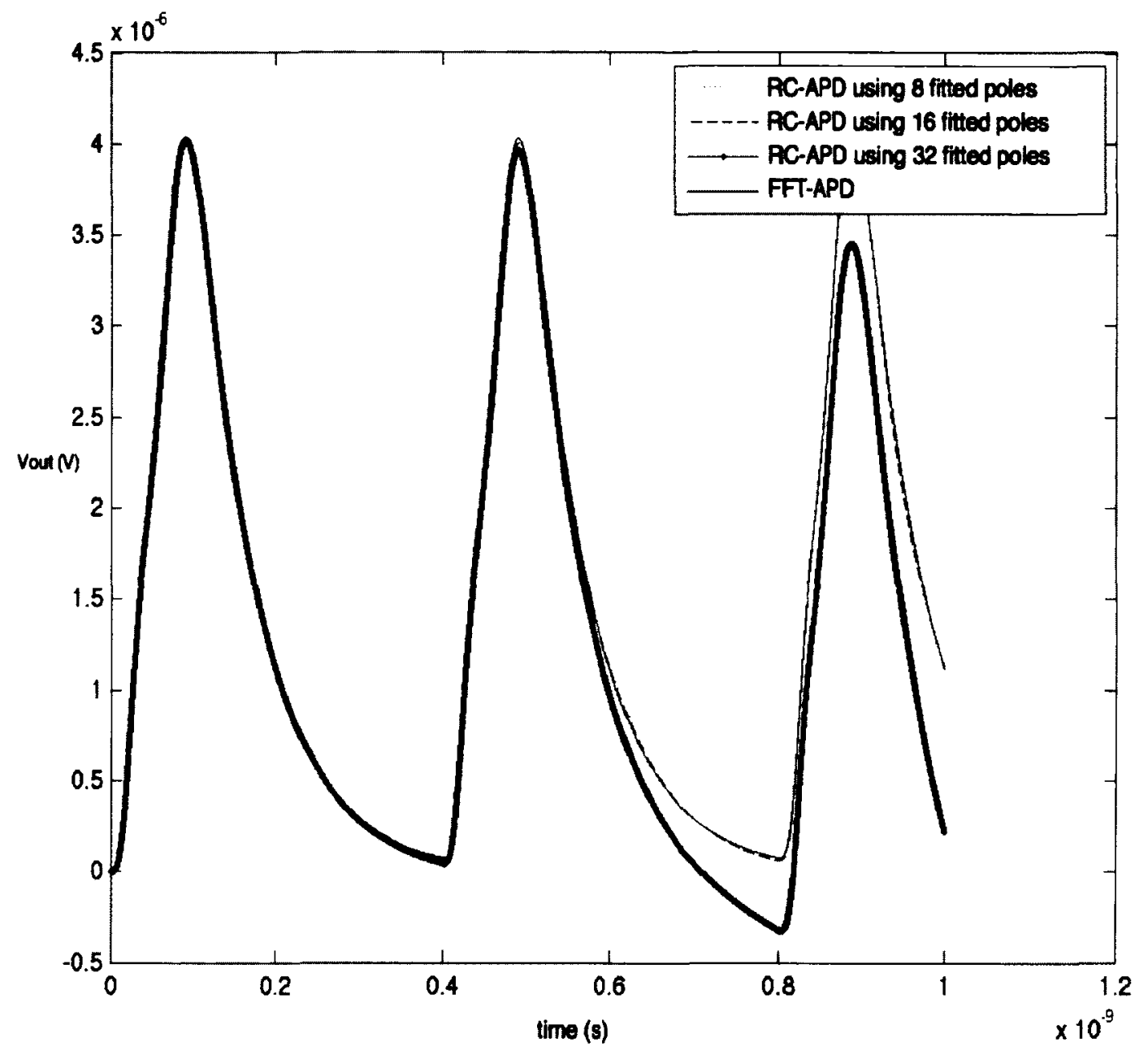

Figure 5.5: RC-APD response for different number of fitted poles

Increasing the number of fitted poles to 16 improves the accuracy of the time domain response as shown in Figure 5.5. Increasing the number of fitted poles to 32 will cause the VF to generate a model with passivity problem. The model reflected this problem in the time domain response as shown in Figure 5.5, (see Chapter 4). 
In Figures 5.6, 5.7 and 5.8, 2 or more segments are required to get a reasonable accurate time domain response using QC-APD macromodel.

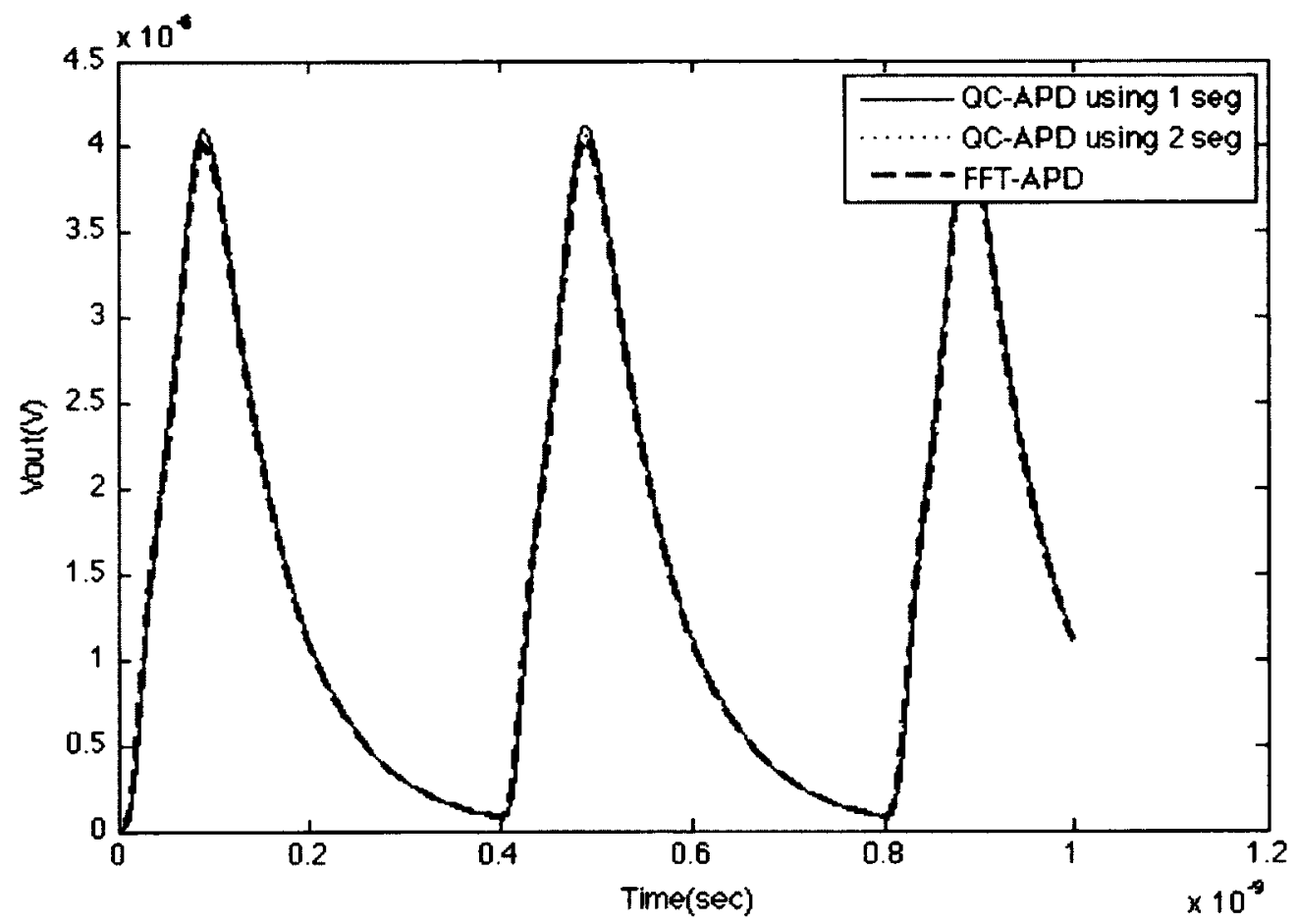

Figure 5.6: QC-APD response for different number of segments 
Therefore $N(t)$ can accurately be approximated by at least 2 segments.

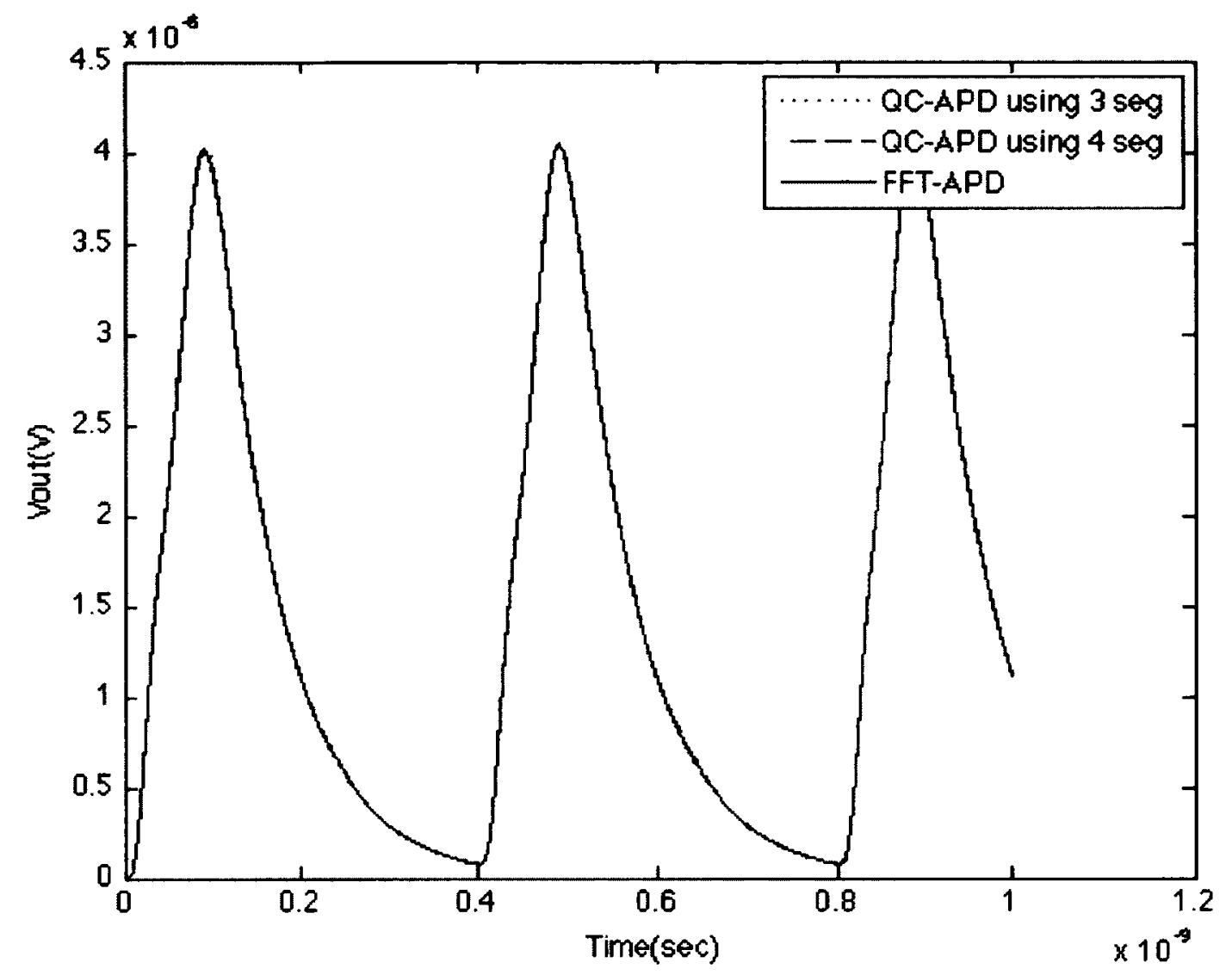

Figure 5.7: QC-APD response for different number of segments

In more sophisticated APDs, the $N(t)$ would need to be remodelled to included more effects. These effects, if dominant would create more variation in the $N(t)$ response. As a result of more variation in the $N(t)$ response, QC-APD macromodel would require more number of segments to accurately replicate the time domain response of the APD. 


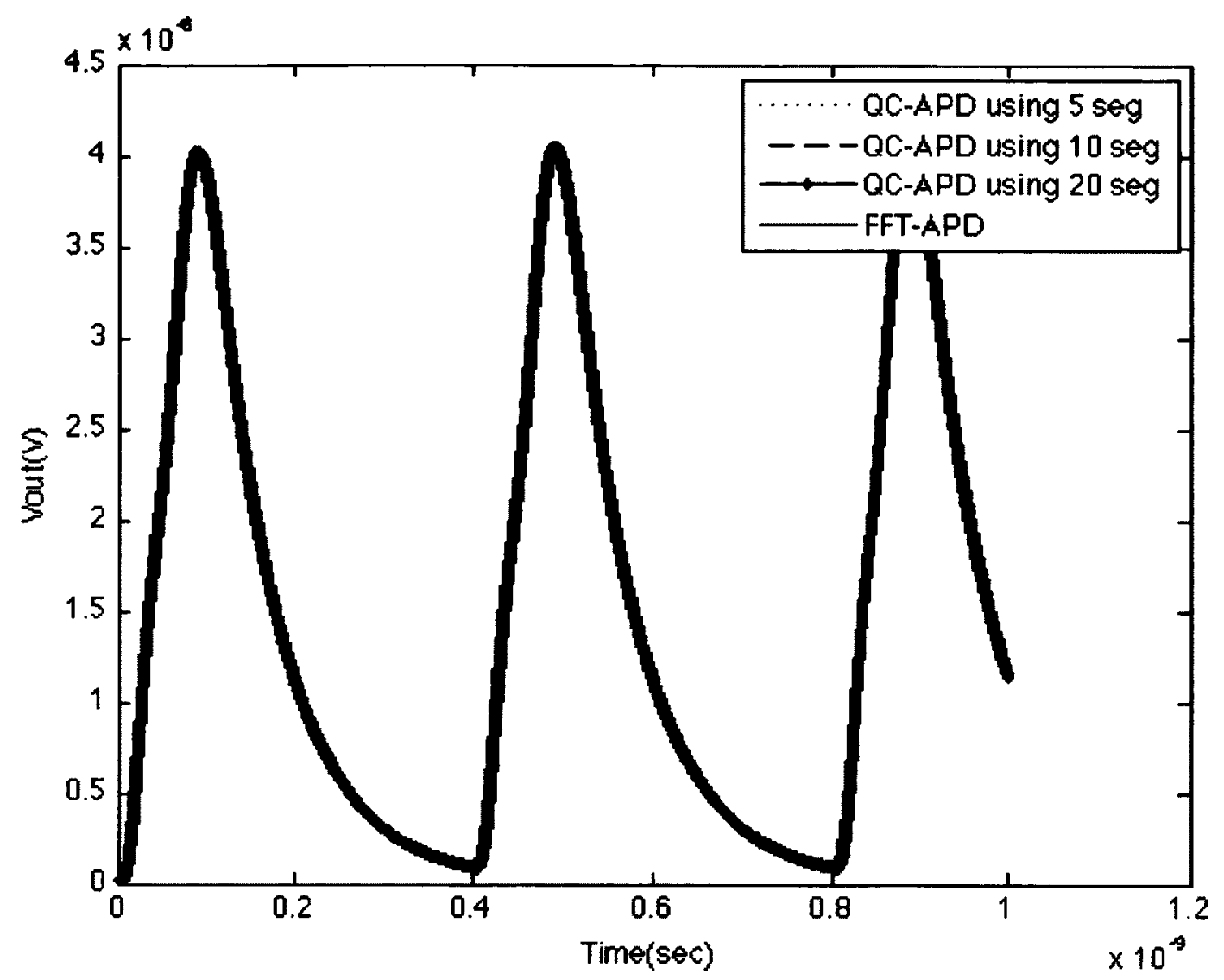

Figure 5.8: QC-APD response for different number of segments

The QC-APD and RC-APD showed similar accuracy. It was noticed that a small accuracy improvement by RC-APD comes at a cost of the macromodel development and validation. This accuracy improvement is outweighed by the effort required for the macromodel development of RC-APD or FFT-APD. Thus, if the user wants to simulate the SAM-APD, then QC-APD is the preferred macromodel because development effort is negligible.

The next example that is presented is a time domain simulation of a circuit with 
an APD that is cascaded to a shunt feedback TIA.

\subsection{Circuit Simulation of an APD terminated with Shunt Feedback TIA}

The example in this section compares the accuracy and simulation processing time of the circuit simulation of an APD that is connected to a shunt feedback TIA as shown in Figure 5.9. The APD model parameters are kept the same as the previous example. An additional simulation will be performed for an IDEAL-APD that is connected to a shunt feedback TIA. The simulation processing time of the IDEAL-APD with the feedback TIA termination will be equivalent to the simulation processing time of shunt feedback TIA. The simulation processing time of TIA feedback is used to normalize the simulation processing time of each of the other three macromodel. Note that the IDEAL-APD refers to a constant current source and its contribution to simulation processing time is almost zero. 


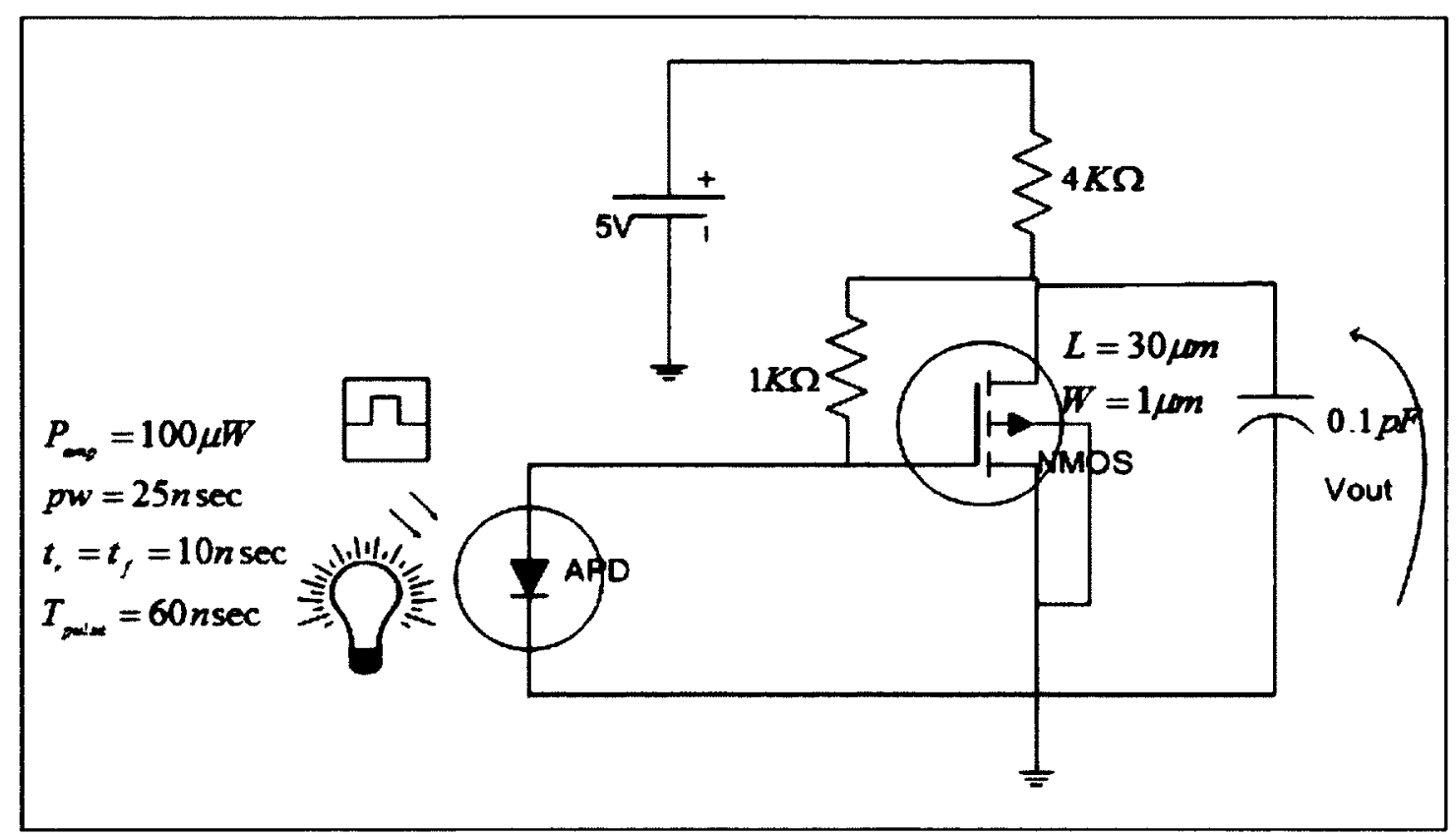

Figure 5.9: Circuit topology of APD with shunt feedback TIA

The computer hardware used to perform simulation processing time measurement is the following:

\begin{tabular}{|c|c|}
\hline Processor & $2.8 \mathrm{GHz}$ Intel Core 2 Duo \\
\hline Type & iMac 7.1 \\
\hline Number of processor & 1 \\
\hline Total number of core & 2 \\
\hline L2 Cache & $4 \mathrm{MB}$ \\
\hline Bus Speed & $800 \mathrm{MHz}$ \\
\hline Memory & $2 \mathrm{~GB} 667 \mathrm{MHz}$ DDR2 SDRAM \\
\hline
\end{tabular}

Table 5.7: Computer hardware used in simulation processing time measurements 


\subsubsection{Simulation Processing Time Results}

The total simulation processing time of the entire circuit versus the number of time points in the simulation is shown in Figure 5.10. As can be seen, the simulation time increases in the order: IDEAL-APD, RC-APD, FFT-APD and QC-APD. In this example, QC-APD has the longest simulation time as the number of time points increase.

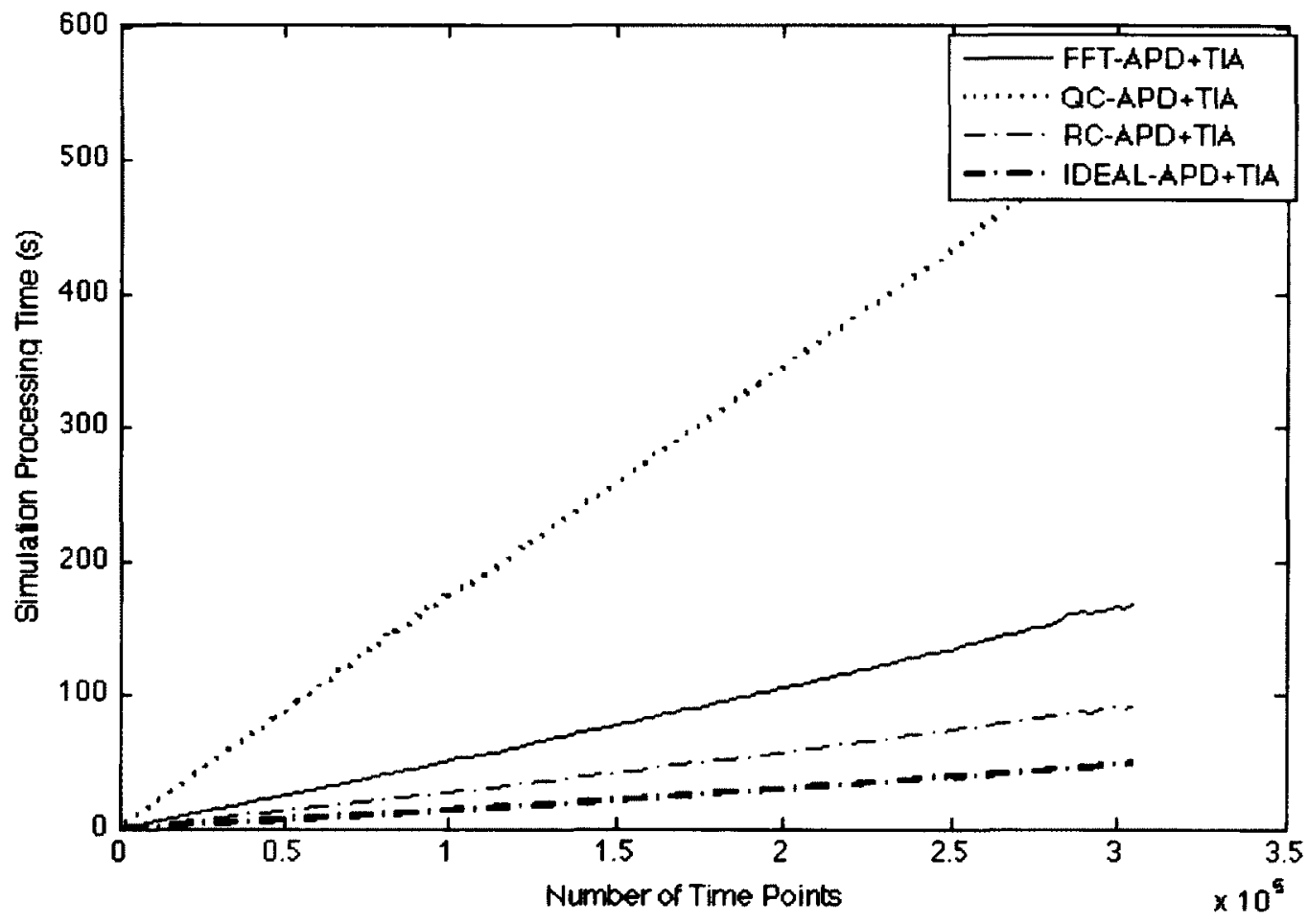

Figure 5.10: simulation processing time of All the three APD models(with TIA) and TIA alone

The reason why FFT APD requires less simulation time is because the input sig- 
nal is not varying quickly and large time steps can be taken; therefore relatively few FFT operations are performed. While QC-APD has to integrate across the entire simulation time and thus requires more simulation processing time. In order to summarize these results, Tables 5.8 and 5.9 describe the speedup of each method and the percentage of processing simulation time taken by the model itself.

\begin{tabular}{|c|c|c|c|}
\hline Method 1 & Method 1:RC-APD & Method 1:QC-APD & Method 1:FFT-APD \\
\hline FFT-APD & 2.5 & $<1$ & 1.0 \\
\hline QC-APD & 12 & 1.0 & 4.5 \\
\hline RC-APD & 1.0 & $<1$ & $<1$ \\
\hline
\end{tabular}

Table 5.8: APD macromodel speedup comparison

The simulation example where APD macromodel is terminated to a nonlinear load has two major contributions to the total simulation processing time. The first contribution is the simulation processing time processing from APD macromodel and the second is from the simulation processing time of the nonlinear termination. In order to compare each APD macromodel, we can compare their contributions to the total simulation processing time. The simulation processing time contribution of an APD macromodel $\left(\% T_{A P D}\right)$ is given below.

$$
\% T_{A P D}=\frac{T_{t o t a l}-T_{N L}}{T_{\text {total }}} \cdot 100
$$

where:

$T_{\text {total }}$ is the total simulation processing time. 
$T_{N L}$ is the total simulation time of the nonlinear load.

Eqn. (5.2) assumes that the simulation processing time of nonlinear load is independent of the APD macromodel. The simulation processing time contribution of each APD macromodel to the total simulation processing time is given in the table below.

\begin{tabular}{|c|c|}
\hline Macromodel & Percentage(\%) \\
\hline FFT-APD & 72 \\
\hline QC-APD & 92 \\
\hline RC-APD & 50 \\
\hline
\end{tabular}

Table 5.9: Comparing simulation processing time contribution of each APD macromodel

It is noticed that FFT-APD macromodel takes 72 percent of simulation processing time. The QC-APD takes about 92 percent of the simulation processing time while RC-APD macromodel takes around 50 percent of the simulation processing time.

The overall result can show that the RC-APD macromodel has consistently the smallest simulation processing time as compared to QC-APD and FFT. The macromodel accuracy comparisons are shown in the next subsection.

\subsubsection{Accuracy of Models}

Initially the APD time domain response using RC-APD, QC-APD and FFT-APD are overlapping each other as shown in Figure 5.11. 


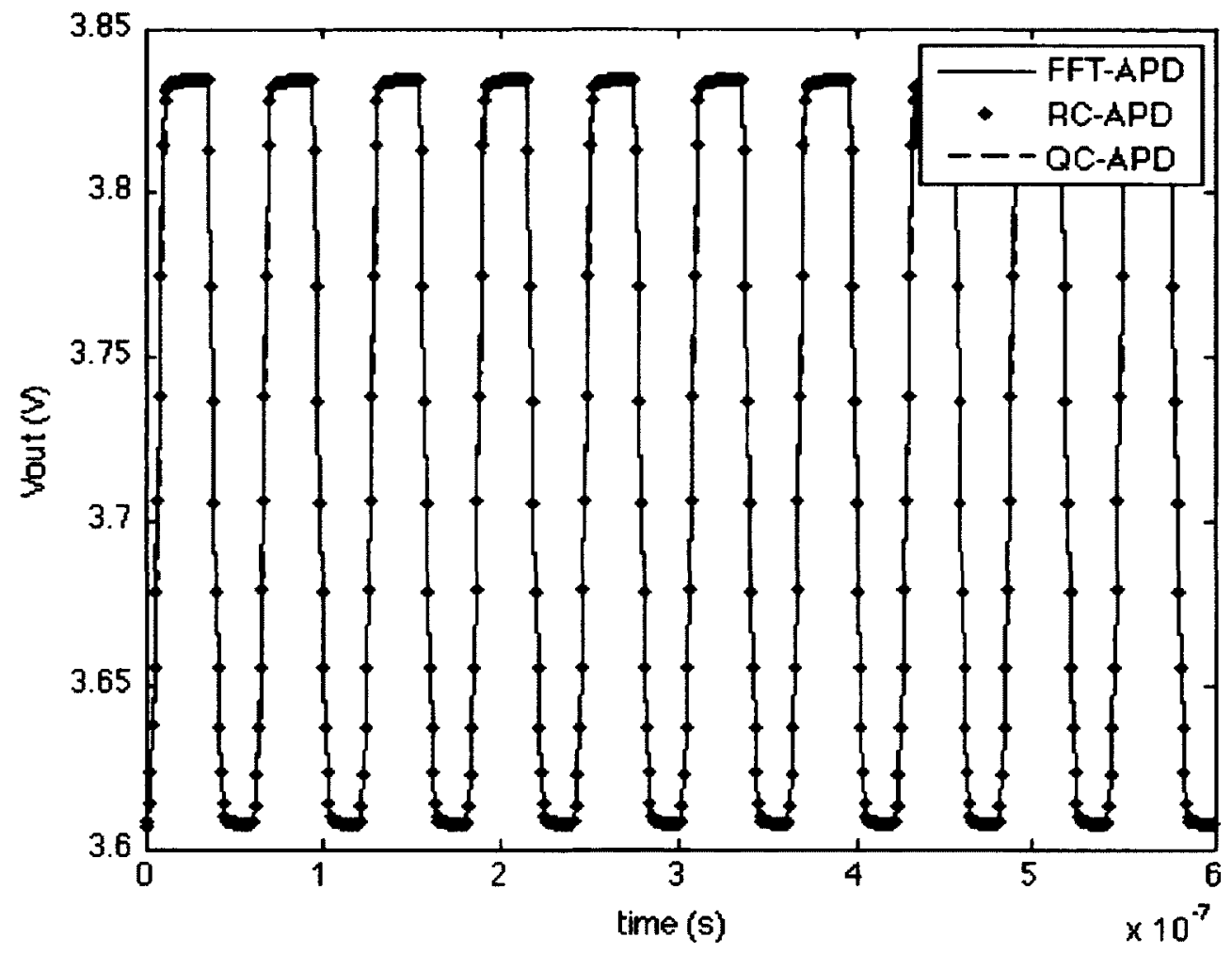

Figure 5.11: The response of the APD FFT-APD, QC-APD and RC-APD model with TIA load

If the number of fitted poles and number of segments are changed, the change in time domain response is shown in Figure 5.12, 5.13, 5.14, 5.15 and Figure 5.16.

In Figure 5.12, the APD with 2 fitted poles does not reach the same maximum as FFT-APD; with 4 fitted poles it slightly over shoots the maximum. 


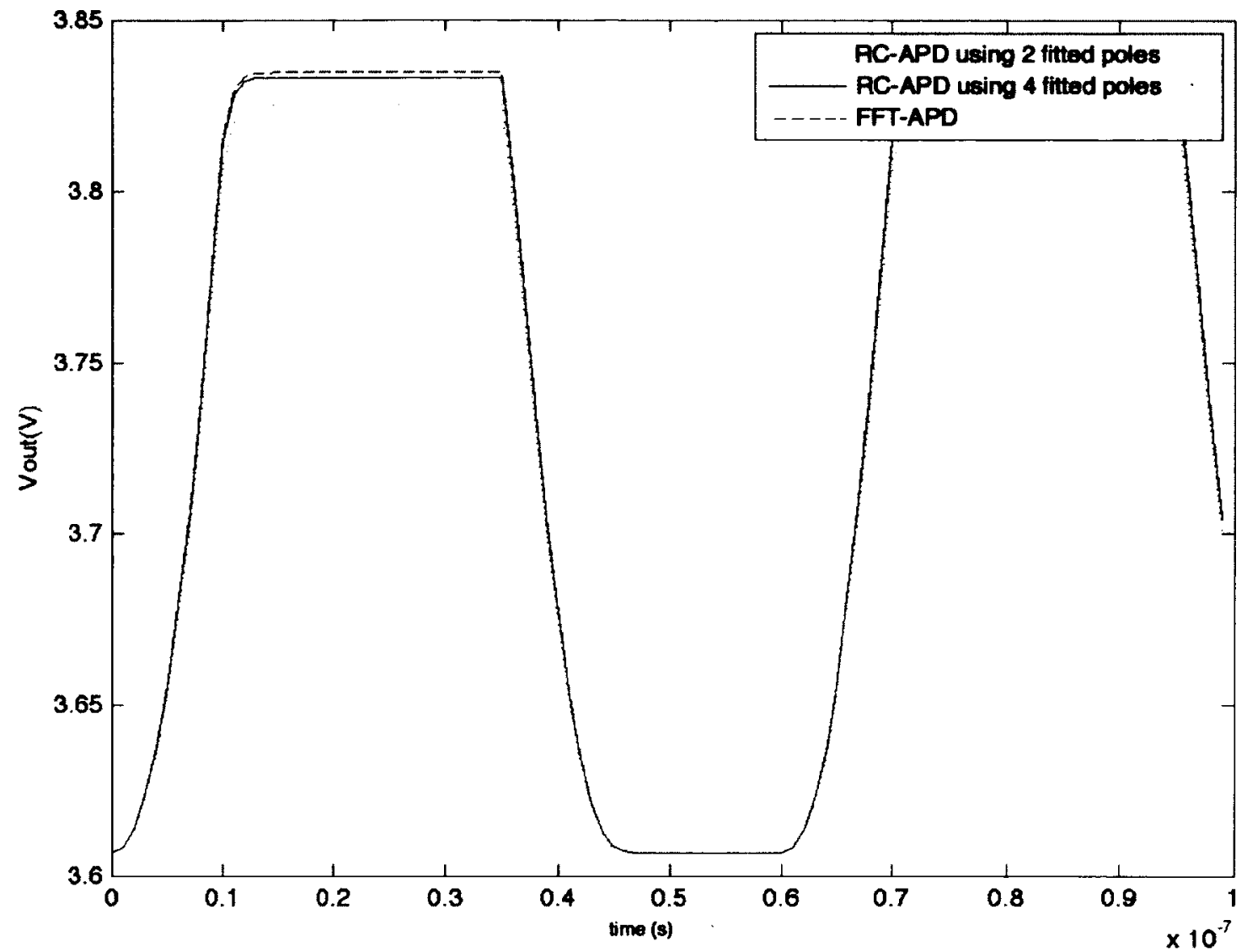

Figure 5.12: RC-APD response for 2 and 4 fitted poles is compared with FFT-APD response

In Figure 5.13, the RC-APD with 8 fitted poles and 16 fitted poles match with FFT-APD. If 20 of fitted poles is used in RC-APD, the Vector Fit's resulting model will have greater fitting error and the passivity problem will appear as shown in Figure 5.13 (see Chapter 4 for details). The next step is to compare RC-APD with QC-APD. 


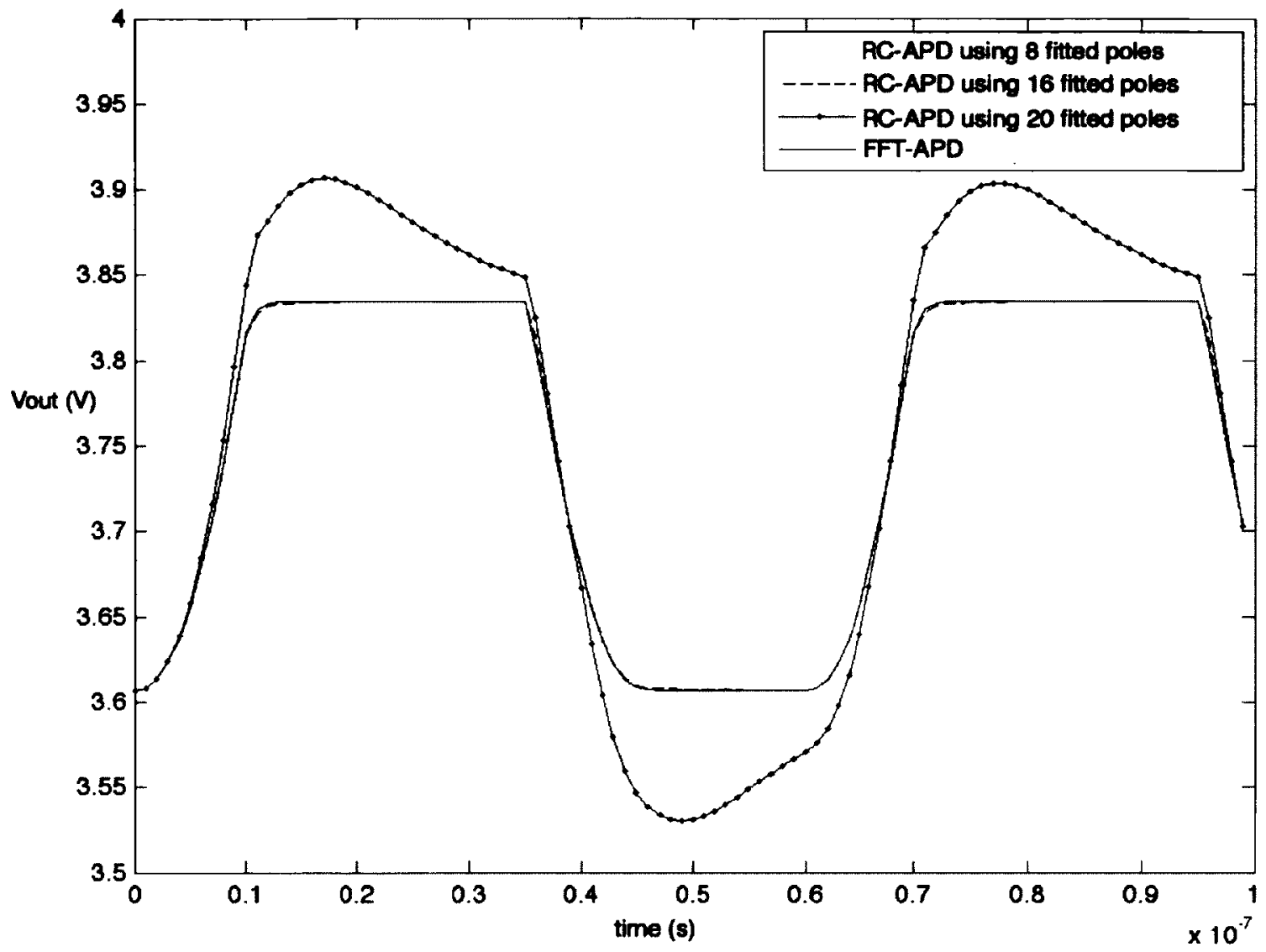

Figure 5.13: RC-APD response for 8, 16 and 20 fitted poles is compared with FFTAPD response 
In Figure 5.14, the QC-APD with 1 segment overshoots the maximum voltage output while with 2 segments it still overshoots the maximum voltage output but it is much closer to the expect FFT-APD response.

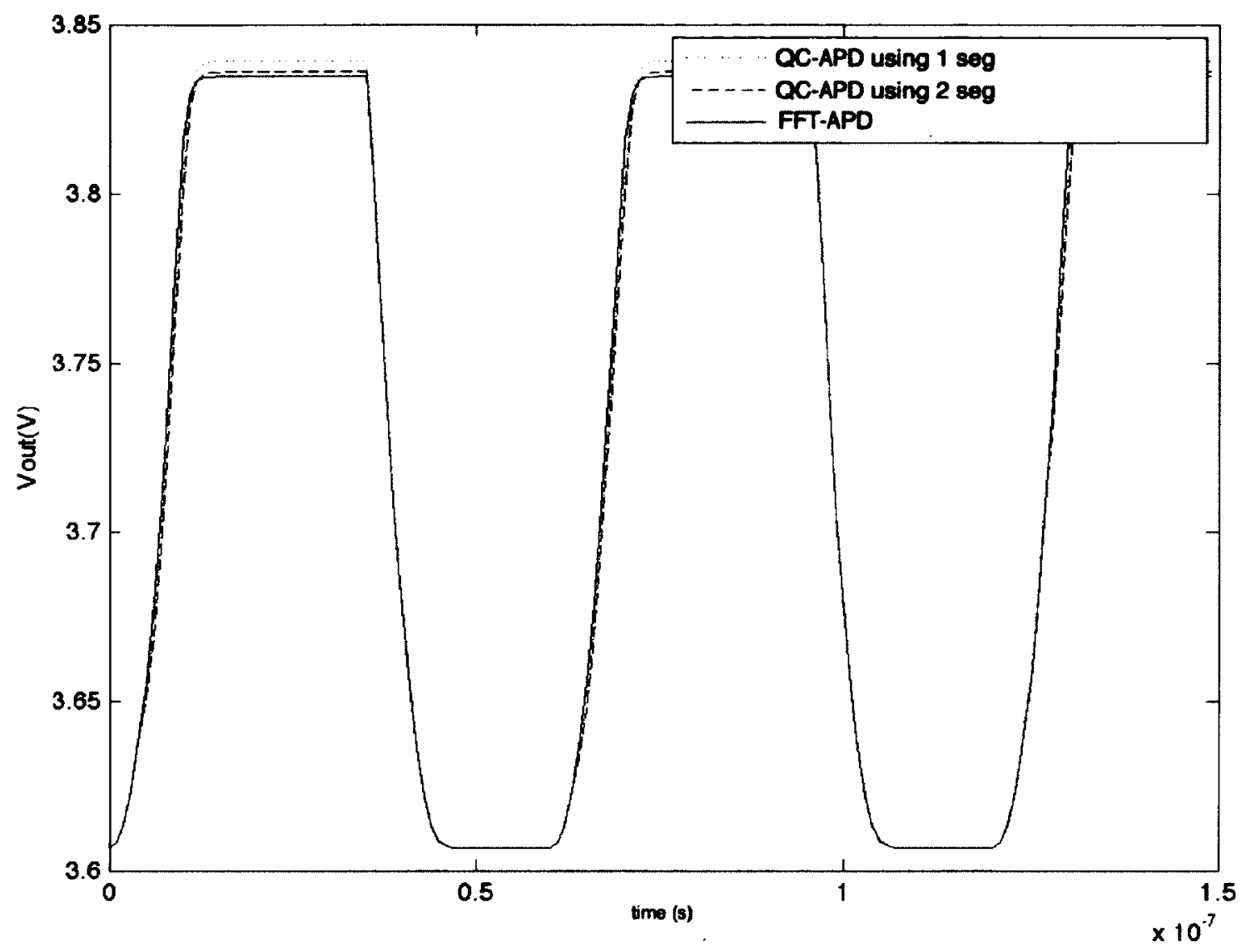

Figure 5.14: QC-APD response for 1 and 2 segments is compared with FFT-APD response

In QC-APD is the segments are increased to 3 or 4 segments the accuracy improves as shown in Figure 5.15. 


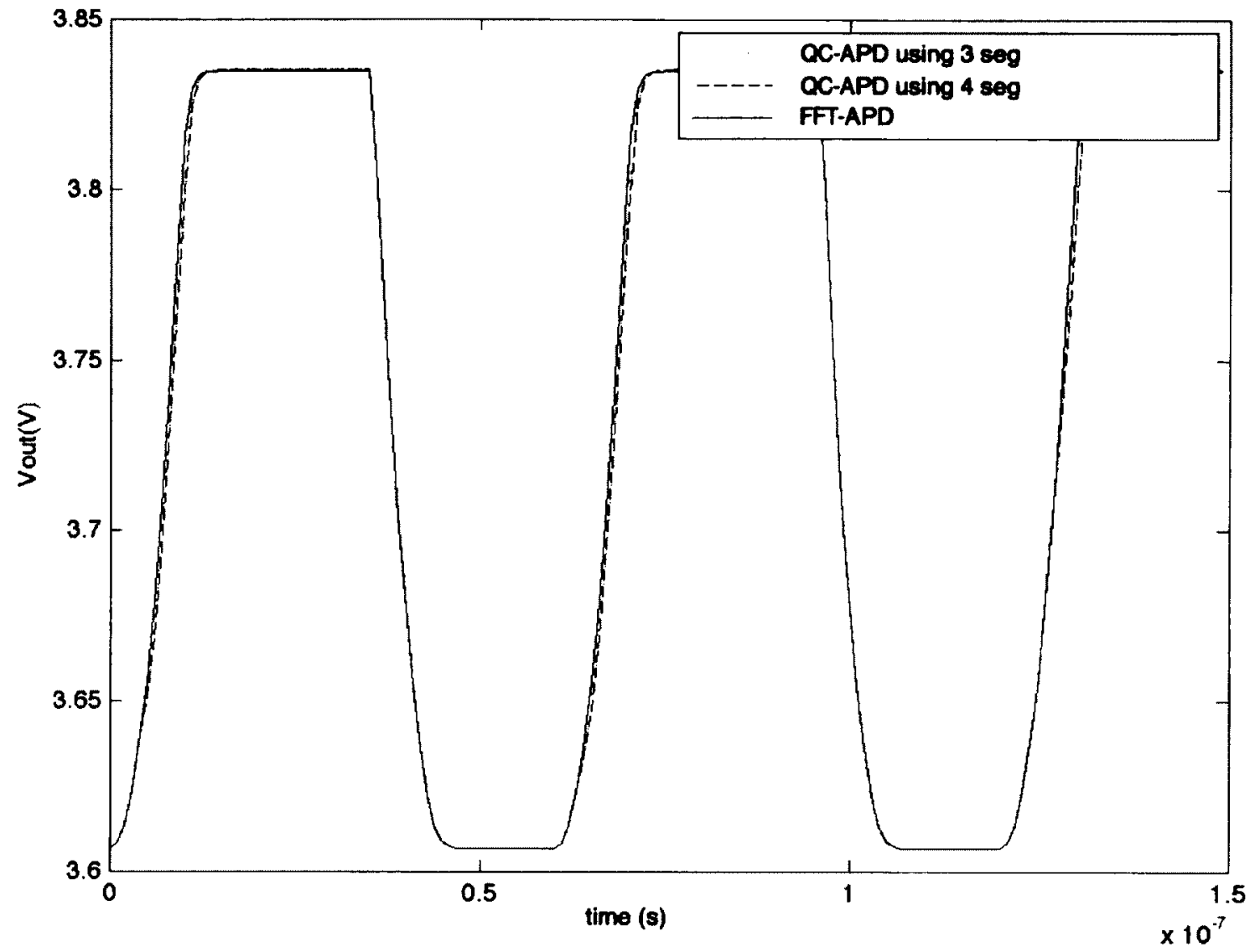

Figure 5.15: QC-APD response for 3 and 4 segments is compared with FFT-APD response

If the segments are increased to 5,10 or 20 , the time domain response becomes even more accurate as shown in Figure 5.16. 


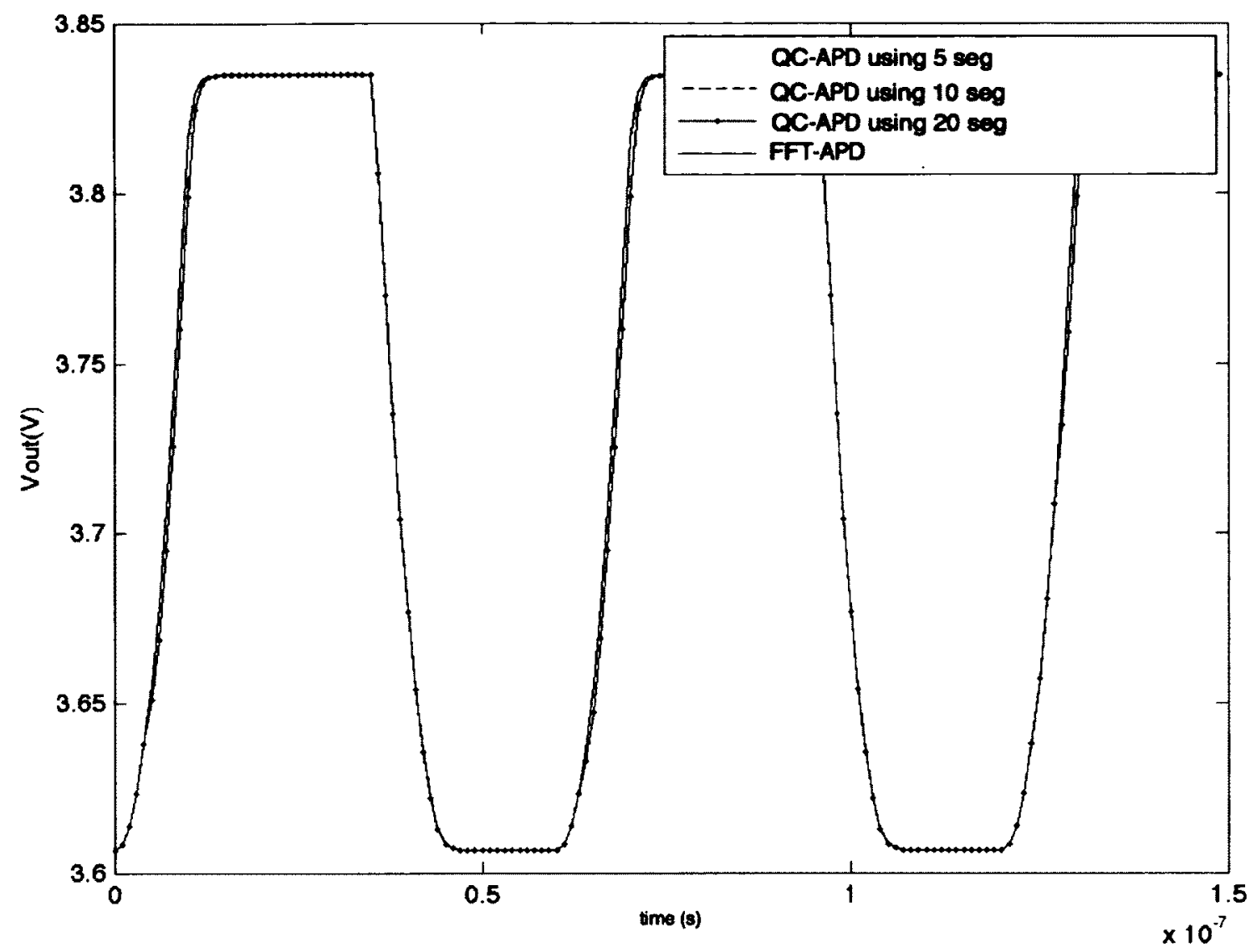

Figure 5.16: QC-APD response for 5,10 and 20 segments is compared with FFT-APD response

Once again, for this example the RC-APD requires more development effort to reach the same level of accuracy as QC-APD. Therefore QC-APD is the preferred macromodel. The next section will review implementation issues of each model. 


\subsection{Macromodel Reliability and Implementation}

\section{Issues}

In this section the reliability of the macromodels is tested and their implementation issues are presented. In order to test the reliability of each model, multiple simulation examples are generated. The examples focus on the variation of the input signal speed, the physical parameters $\alpha, v_{n}$ and the nonlinear load to confirm consistency. The circuit topology of the simulation examples is shown in Figure 5.17.

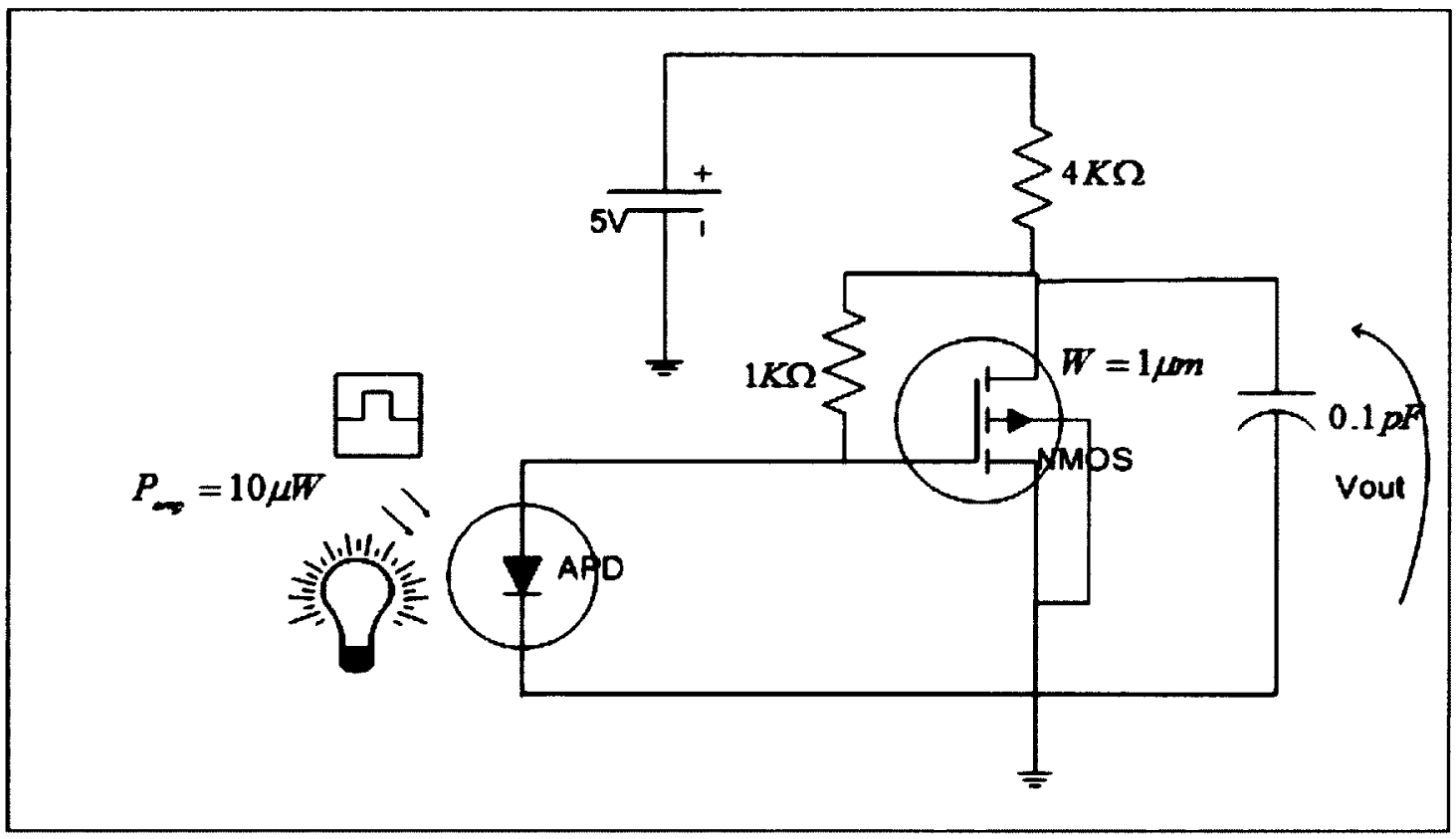

Figure 5.17: Circuit topology APD and TIA for macromodel reliability testing

The constant APD model and macromodel parameters are shown in Tables 5.10, $5.11,5.12$ and 5.13. 


\begin{tabular}{|c|c|}
\hline Parameter Names & Values \\
\hline \hline$P D_{\text {eff }}$ & 0.5 \\
\hline$W_{a}$ & $2.0 \mu \mathrm{m}$ \\
\hline $\mathrm{W}$ & $2.7 \mu \mathrm{m}$ \\
\hline$x_{m}$ & $0.5 \mu \mathrm{m}$ \\
\hline$x_{t}$ & $0.7 \mu \mathrm{m}$ \\
\hline$x_{a}$ & $2.7 \mu \mathrm{m}$ \\
\hline$M_{0}$ & 3 \\
\hline $1 / e_{h}$ & $4 \cdot 10^{-12} \mathrm{~s}$ \\
\hline $1 /\left(2 \pi \tau_{m}\right)$ & $60 \mathrm{GHz}$ \\
\hline$L$ & $0.1 \mathrm{nH}$ \\
\hline$C$ & $0.1 \mathrm{pF}$ \\
\hline$R$ & $60 \Omega$ \\
\hline$v_{p}$ & $4.8 \cdot 10^{6} \mathrm{~cm} / \mathrm{s}$ \\
\hline
\end{tabular}

Table 5.10: Constant physical parameters for implementation issues testing

\begin{tabular}{|c|c|}
\hline Parameter Name & Value \\
\hline Number of Segments & 20 \\
\hline
\end{tabular}

Table 5.11: Example 1:QC-APD model parameters

\begin{tabular}{|c|c|}
\hline Parameter Name & Value \\
\hline Frequency Range & $0-300 \mathrm{GHz}$ \\
\hline Fstep & $73.26 \mathrm{MHz}$ \\
\hline
\end{tabular}

Table 5.12: FFT-APD model parameters

\begin{tabular}{|c|c|}
\hline Parameter Name & Value \\
\hline Number of Fitted Poles & 16 \\
\hline Range of Poles Values (logarithmically spaced) & $10^{7}-10^{12} \mathrm{~Hz}$ \\
\hline Frequency Range & $0-300 \mathrm{GHz}$ \\
\hline Fstep & $73.26 \mathrm{MHz}$ \\
\hline
\end{tabular}

Table 5.13: RC-APD model parameters

The simulation examples for reliability testing use a fast and a slow train of pulses 
as specified in Table 5.14 .

\begin{tabular}{|c|c|c|c|c|c|}
\hline Type & $P_{a m p}$ & $t_{r}$ & $t_{f}$ & $p w$ & T \\
\hline Fast Pulse & $10 \mu W$ & $3 \mathrm{psec}$ & $3 \mathrm{psec}$ & $6 \mathrm{psec}$ & $1.5 \mathrm{nsec}$ \\
\hline Slow Pulse & $10 \mu W$ & $2 \mu \mathrm{sec}$ & $2 \mu \mathrm{sec}$ & $4 \mu \mathrm{sec}$ & $15 \mu \mathrm{sec}$ \\
\hline
\end{tabular}

Table 5.14: Table describing the fast and the slow pulse

Note that for each of the inputs, the APD model parameters $\alpha, v_{n}$ and the shunt feedback gate lengths as shown in Table 5.15, are varied in permutation in order to test macromodel reliability.

\begin{tabular}{|c|c|c|}
\hline Parameter Name & Symbol & Values \\
\hline Electron saturated drift Mobility $(\mathrm{m} / \mathrm{sec})$ & $v_{n}$ & $63000,70000,77000$ \\
\hline Absorption Coefficient $\left(\mathrm{m}^{-1}\right)$ & $\alpha$ & $920000,1150000,1380000$ \\
\hline TIA's NMOS Transistor Gate Length $(\mu \mathrm{m})$ & $\mathrm{L}$ & $10,30,50$ \\
\hline
\end{tabular}

Table 5.15: Variable simulation parameter for testing macromodel reliability

\subsubsection{Macromodel Reliability Check with Fast Input Pulses}

In this subsection, the input pulse is kept constant with a fast pulse but APD parameters and transistor gate length are varied to check the macromodel consistency.

The time domain responses of FFT-APD, QC-APD and RC-APD macromodels, with model parameters $v_{n}=77 \cdot 10^{3}\left(\frac{m}{s}\right), \alpha=1.15 \cdot 10^{6}\left(\mathrm{~m}^{-1}\right)$, and the NMOS gate length of $50 \mu m$ are shown in Figure 5.18.

All three macromodels show good accuracy because the time domain response for this example are all overlapping. 


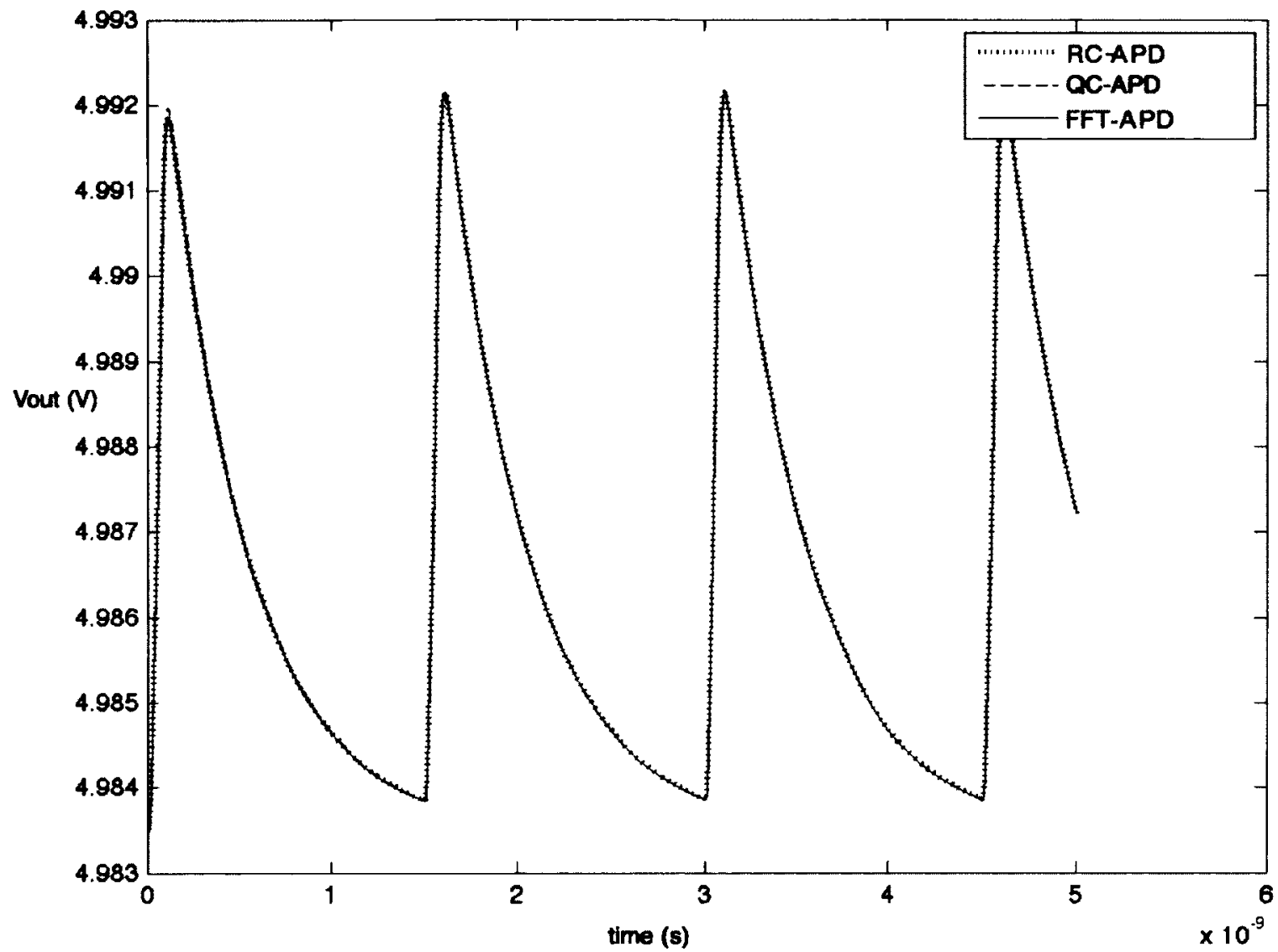

Figure 5.18: The time domain responses of FFT-APD, QC-APD and RC-APD macromodels,

The time domain responses of FFT-APD, QC-APD and RC-APD macromodels, with model parameters $\alpha=1.38 \cdot 10^{6}\left(\mathrm{~m}^{-1}\right), v_{n}=63 \cdot 10^{3}\left(\frac{\mathrm{m}}{\mathrm{s}}\right)$, and the NMOS gate length of $10 \mu m$ are shown in Figure 5.19. 


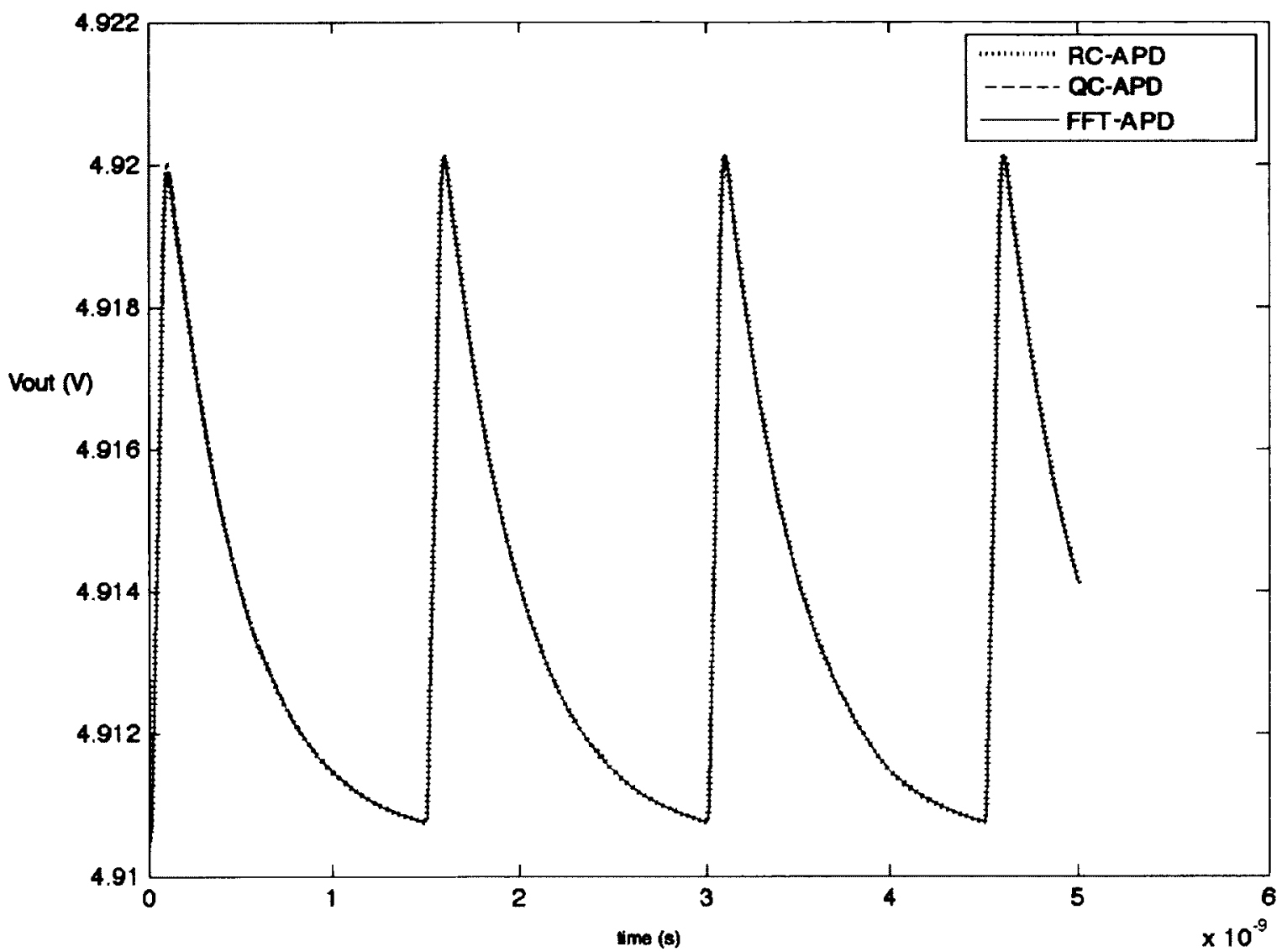

Figure 5.19: Time domain response of FFT-APD,RC-APD and QC-APD

In Figure 5.19, QC-APD and FFT-APD and RC-APD overlap each other.

A large number of simulations were performed confirming these results (see Appendix B for examples.)

The next section will repeat the same set of simulation experiments with a slower pulse input. 


\subsubsection{Reliability Check for Slow Input}

As in the previous section the macromodel reliability check will be performed for all the permutations but only a small subset of responses will be presented in detail. The time domain response of FFT-APD, QC-APD and RC-APD macromodels are shown in 5.20 where the $\alpha=92 \cdot 10^{5}\left(\mathrm{~m}^{-1}\right)$ and $v_{n}=70 \cdot 10^{3}\left(\frac{\mathrm{m}}{\mathrm{s}}\right)$ and the NMOS gate length is $l=50 \mu m$. Both APD macromodels show consistent accuracy as shown in Figure 5.20 . 


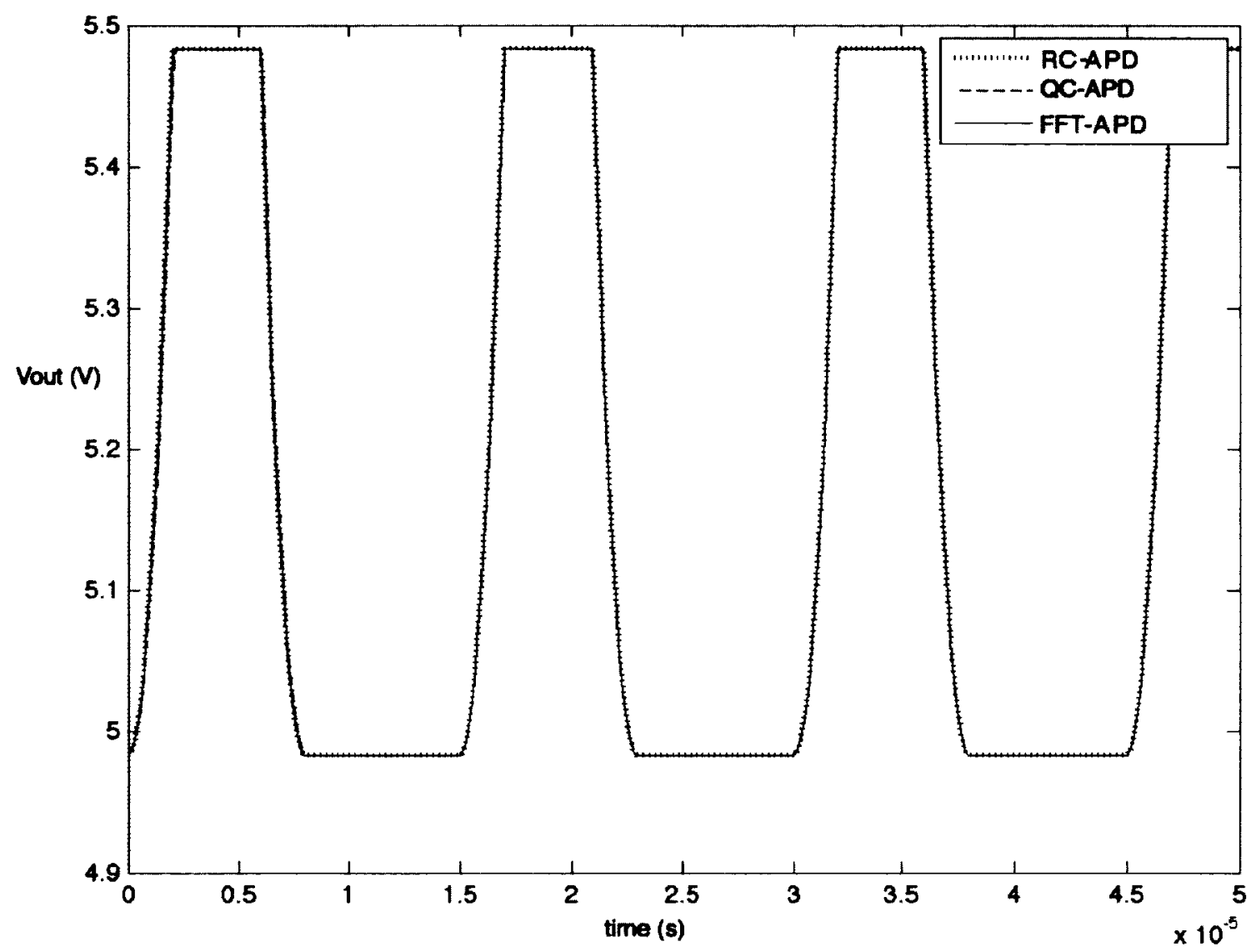

Figure 5.20: Time domain response of FFT-APD, RC-APD and QC-APD

A large number of simulations were performed confirming these results see (Appendix B for examples.)

The next subsection will present the effect of time step control on the simulation processing time and accuracy of RC-APD and QC-APD. 


\subsection{Effects of Simulation Step Size Control on APD}

\section{Models}

In this section we will compare the simulation responses of RC-APD and QC-APD with uniform step size simulation. Then we will compare the number of simulation time points versus the input rise time of QC-APD simulation examples with those of RC-APD. These simulation examples were done using nonuniform step size simulation.

\subsubsection{Uniform Step Size Simulation}

The time domain responses of RC-APD and QC-APD with the step size fixed are shown in Figures 5.21 and 5.22.

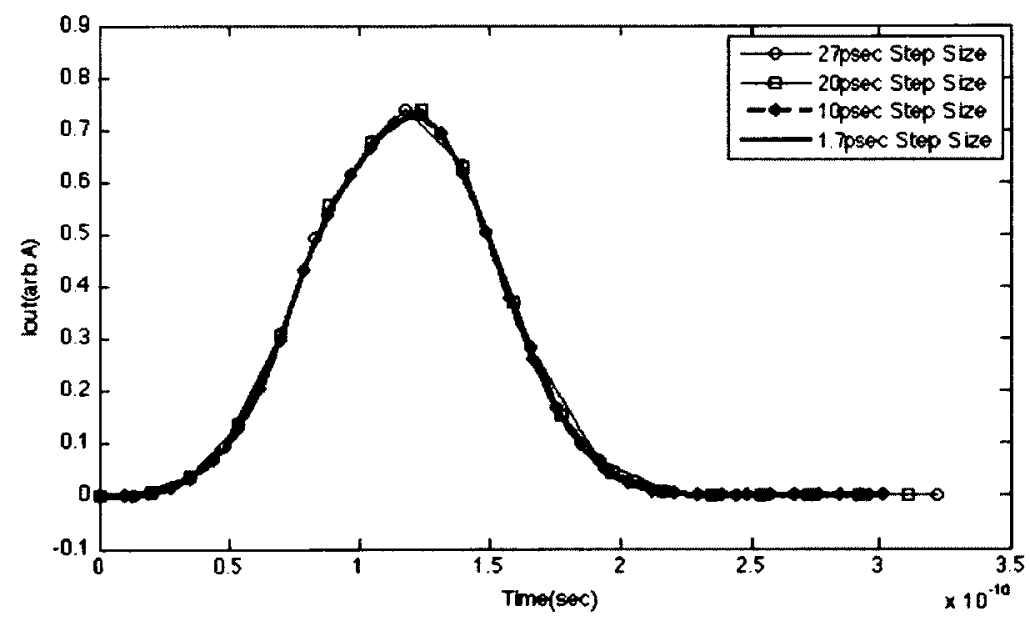

Figure 5.21: Time domain responses of RC-APD with different uniform simulation time step 


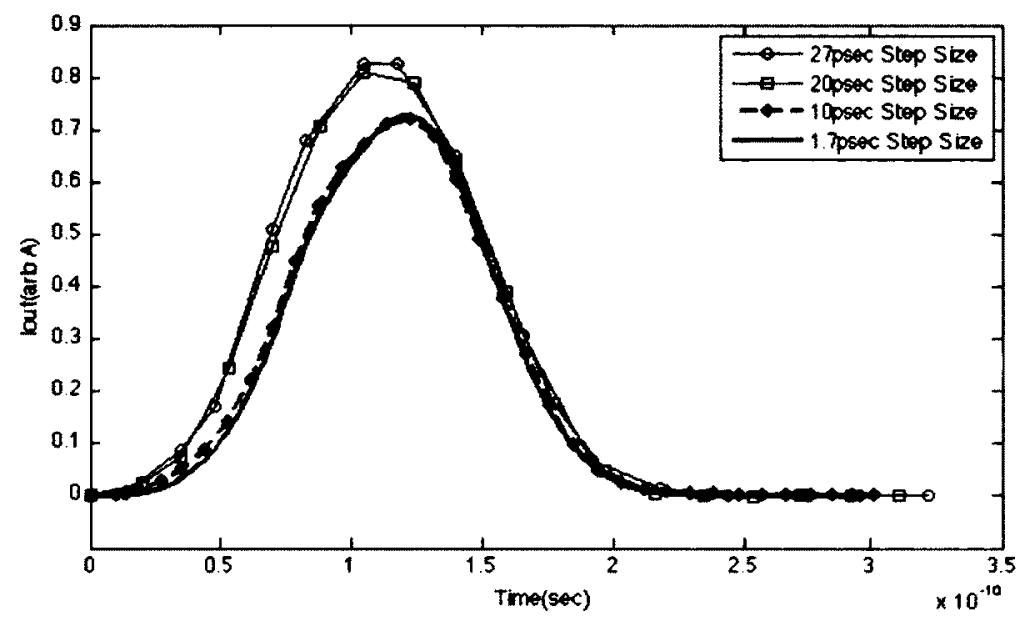

Figure 5.22: Time domain APD response of QC-APD with different uniform simulation time step

For QC-APD, if the step size is large with respect to the segment size, segments can get skipped and thus accuracy is lost. As the number of time points is increased, the accuracy is improved as each segment has an effect on the output. The RC-APD is unaffected by large or small step sizes.

\subsubsection{Simulation Example with Non Uniform Simulation Step}

\section{Size}

In this section, the QC-APD and RC-APD are going to be compared for the number of time points required to run the same simulation with the same initial step, but varying step size during simulation.

The $\mathrm{QC}-\mathrm{APD}$ requires more time points in the transient regions as compared to 
RC-APD as shown in Figure 5.23. Note that the initial step size is the same and the end time is the same. QC-APD requires more time points because each segment has a well defined duration and this duration affects the maximum step size of the simulation. During periods when the input is varying quickly, simulation step size must be smaller than the time length of each segment; else the segments will be skipped thus introducing error. The RC-APD is defined by poles and residues that are not influenced by the step size.

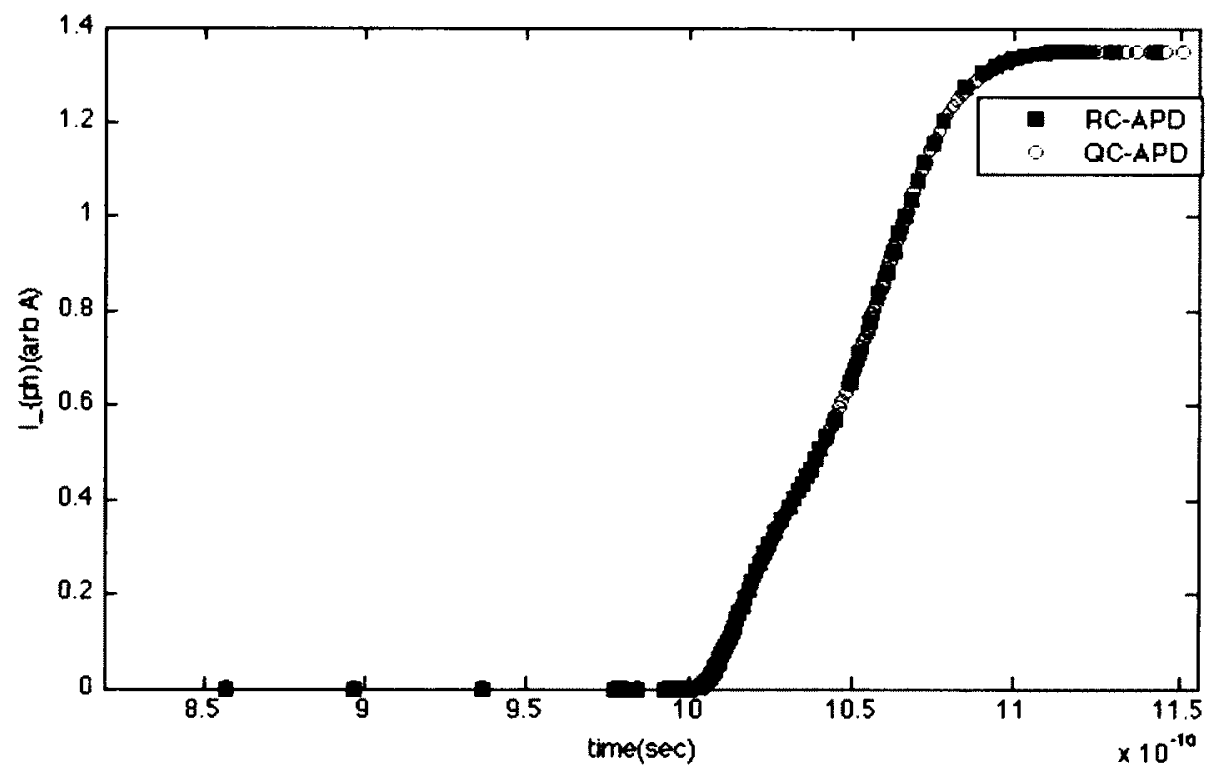

Figure 5.23: Time domain APD response of QC-APD with non uniform time step for a 1 psec rise time input 


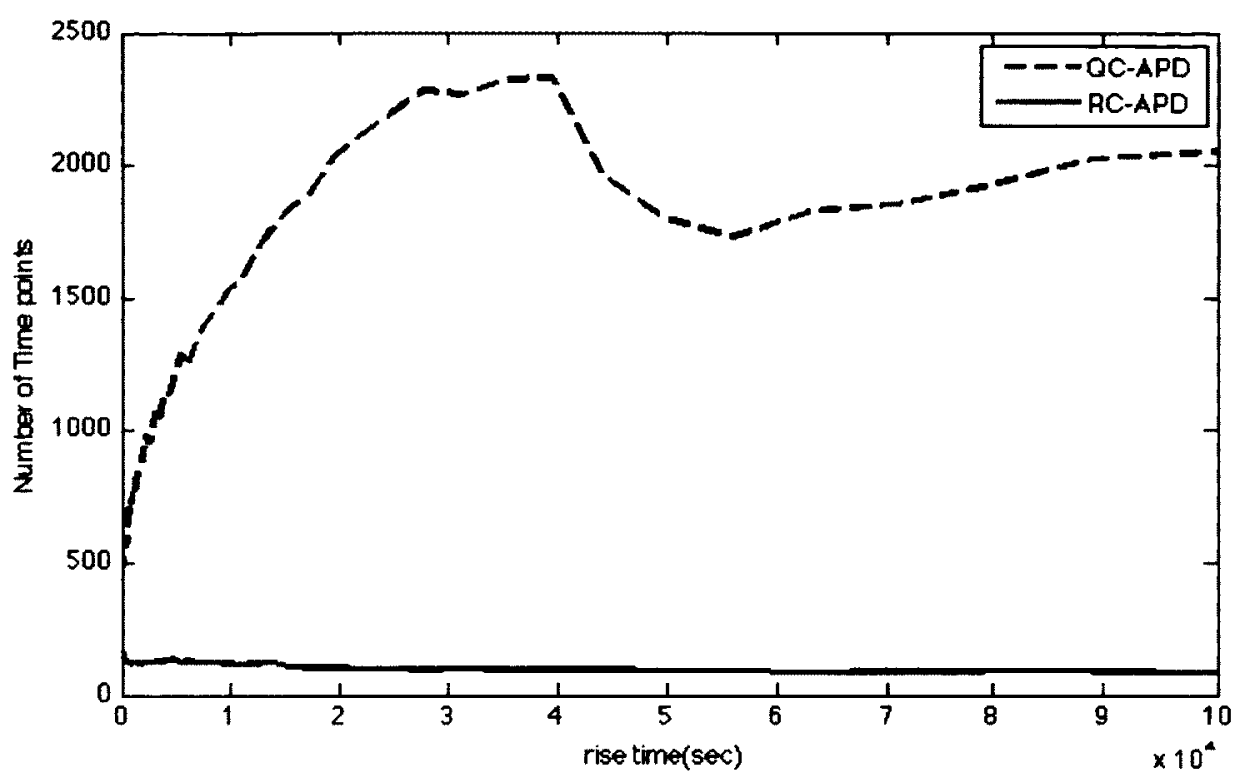

Figure 5.24: Time domain APD current response of QC-APD with non uniform time step for a $1 \mathrm{psec}$ rise time input

As can be seen in Figure 5.24 the number of time steps taken by the QC-APD is complicated. It is determined both by the rate at which the input varies and the duration of variation. Initially, as the rise time increases the duration of the pulse edge increases and as the time step is determined by the segment size, the number of time steps increase. However, as rise time increases further the variation in the input over a segment time becomes sufficiently small that segments can be skipped with out effecting accuracy or convergence and the number of time steps drops. 


\section{Model Comparisons}

The table below compares the CE-APD, FFT-APD, QC-APD and RC-APD models with respect to stability, speed, accuracy, physical model properties and user input to generate the model and model creation overhead.

The CE-APD model is quick and fast, simple to implement and use, but internally it has a negative resistor that causes the circuit to be unstable for time domain simulations.

The FFT-APD model is accurate and applicable to any APD device with a frequency response. The drawback of this model is the fact that it can be very slow if the input is complex and rapidly changing (requiring short time steps) and therefore the simulation processing time can be long. It also requires a frequency response and is awkward to implement in a SPICE engine. It implicitly assumes periodicity and this assumption can introduce error if the model is not used correctly.

QC-APD will be faster than the FFT-APD for relatively quickly varying input, but can be slower for low bandwidth signals, however, it is very accurate. It is primarily

\begin{tabular}{|c|c|c|c|c|c|}
\hline Model & Stability & Speed & Accuracy & $\begin{array}{c}\text { Physical } \\
\text { Model }\end{array}$ & $\begin{array}{c}\text { Model } \\
\text { Creation Overhead }\end{array}$ \\
\hline \hline CE-APD & Not Stable & N/A & Yes & Yes & No \\
\hline FFT-APD & Stable & Slowest & Yes & No & Yes \\
\hline QC-APD & Stable & Fast & Yes & Yes & No \\
\hline RC-APD & Stable & Fastest & Yes & No & Yes \\
\hline
\end{tabular}

Table 5.16: Comparing APD models 
a physical model and can be easily implemented in SPICE. This model requires little or no user input to adjust accuracy and speed of the model. It is easy to modify the physical parameters of this model.

However, it is only valid for the given physical geometry. If the geometry changes, the QC-APD would have to be redefined and restructured to model the response of the new geometry. During simulation of QC-APD, convergence issues could arise if the step size is not adequate or fixed. Thus QC-APD restricts time step flexibility. The RC-APD model is the fastest model (small simulation processing time) and quite accurate. It is applicable to any APD geometry with a frequency response derived from a theoretical model or measured data because it is a non physical model. This model requires more overhead during development because the user needs to find the right number of fitted poles. Thus changing model parameters requires re-creation of the model. In order to obtain the poles, the Vector fit algorithm must be used. The accuracy is strongly dependent on the chosen number of poles. 


\section{Chapter 6}

\section{Conclusion and Future Work}

Analyzing the macromodel developments showed that RC-APD macromodel and FFT-APD require pre processing of an APD model data and user intervention. The QC-APD only needs a time domain impulse response of $N(t)$ and built in SPICE elements. The CE-APD is just composed of SPICE elements and thus it is very cheap and easy to develop in SPICE engine but it is not functional as shown in Chapter 3. The QC-APD showed good accuracy and was relatively efficient. Increasing the number of segments increased the accuracy and simulation processing time and reducing the number of segments reduced the accuracy and the simulation processing time. The accuracy of RC-APD is variable; too few poles produce inaccurate results and too many poles also produce inaccurate results. The FFT-APD assumes periodicity which can create issues. The user has to apply an appropriate frequency resolution with 
adequate maximum frequency to avoid aliasing using the FFT-APD macromodel.

The RC-APD macromodel is as much as 57 times faster than FFT-APD macromodel and as much as 10 times faster than QC-APD macromodelfor situations where the time is dominated by input bandwidth.If the bandwidth of the input is low, QCAPD requires more simulation processing time. But FFT-APD requires a smaller number of FFT operations and thus requires a smaller simulation processing time than QC-APD. Therefore the QC-APD speedup drops to below unity. The average percentage of simulation processing time taken by FFT-APD and QC-APD can be almost as much as the simulation processing time of the TIA alone. However, the simulation processing time is much smaller than the simulation processing time of the TIA.

In the case of a non-fixed time step simulation, the number of time steps taken by the QC-APD is complicated. It is determined by the bandwidth of the input pulse. Initially, as the rise time increases, the duration of the pulse edge increases and as the time step is determined by the segment size, the number of time steps increase. However, as rise time increases further the variation in the input over a segment time becomes sufficiently small that segments can be skipped without affecting accuracy or convergence and the number of time steps drops. The RC-APD has no step size constraints and thus consistently has a small number of time steps.

The macromodel accuracy for linear and nonlinear terminations was shown to be 
effective for all three macromodels.

Reliability testing showed that each model can be accurate for a variety of APD parameters, nonlinear loads and different input signals. The root cause of the variation in accuracy for RC-APD is the quality of the fit that was created by VF algorithm which is dependent on the initial pole guess and the number of fitted poles.

The QC-APD and RC-APD showed similar accuracy. It was noticed that a small accuracy improvement by RC-APD comes at a cost of the macromodel development and validation. This accuracy improvement is outweighed by the effort required for the macromodel development of RC-APD or FFT-APD. Thus, if the user wants to simulate the SAM-APD, then QC-APD is the preferred macromodel because development effort is negligible. But if the user wants to simulate an APD structure, which does not have a QC formulation then RC-APD or FFT-APD would have to be used.

RC-APD has shown simulation speedup improvements as compared to QC-APD and FFT-APD. The problem with this speedup benefit is the fact that RC-APD requires more effort for development and validation. But the development and validation could partially be automated to speedup the model creation process. Theoretically, the model creation process is done only once where the vendor of the model can provide the libraries of APD model. In this case the burden of the model creation is removed from the user and the user can focus on the design. 


\subsection{Future Work}

The following aspects would be researched in the future:

1. Development of a passive macromodel from the model obtained from vectorfit using passivity compensation techniques and verifying its effectiveness against FFT-APD and QC-APD

2. Development of voltage controlled parameters for physical APD models or from measurements. In reality, APD are biased at a voltage in order to improve the physical behaviour of the APD model; relating the voltage bias to the APD performance could improve the credibility of the APD model.

3. Development of a passive nonlinear APD time domain macromodel. The current APD model is a small signal model only representing one bias point; in reality APD is a nonlinear device. The APD model must be created to accommodate a change in bias. The APD model should follow the conservation of energy and thus must be passive. 


\section{Appendix A}

\section{Review of TIA}

The transimpedance amplifier (TIA) is an amplifier that amplifies the conversion of the photogenerated current into a voltage. [19] [20]. The shunt feedback TIA, as shown in Figure A.1 (a), will be used in APD simulation examples. Note that A will be a common source amplifier with NMOS technology, as shown in Figure A.1 (b). 

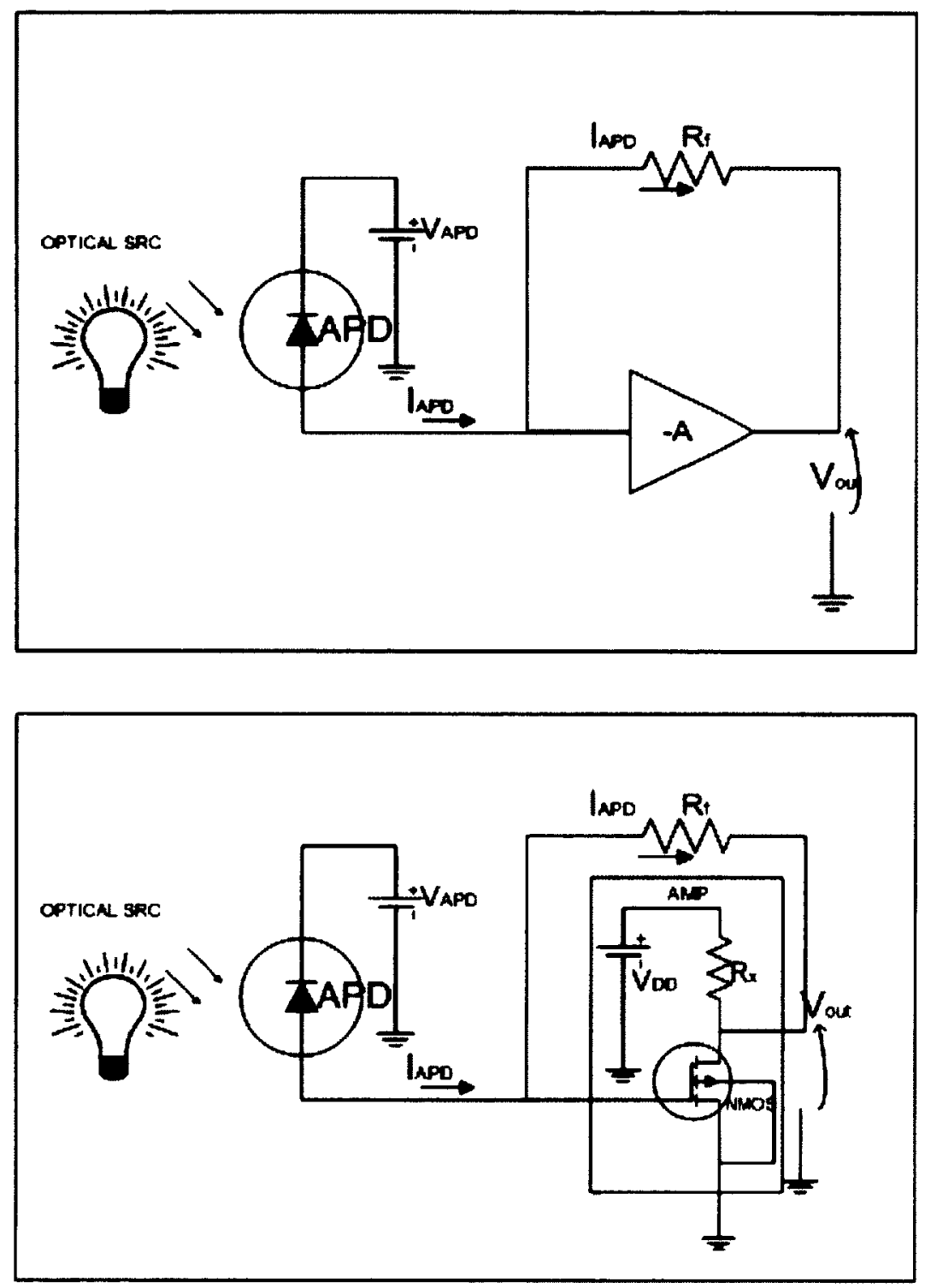

Figure A.1: General shunt feedback TIA (a) and shunt feedback with common source amplifier (b)

The ideal transimpedance gain of shunt feedback TIA of Figure A.1 (b), is given in (A.1) [20] [19]

$$
Z_{\text {gain }}=\frac{A}{A+1} R_{f}
$$


Where $Z_{\text {gain }}$ is the transimpedance gain $(\Omega)$, A is the voltage gain of the common source amplifier, $R_{f}$ is the feedback resistor $(\Omega)$ and $R_{x}$ is a bias resistor $(\Omega)$. The bias of common source amplifier and the APD are independent of each other. 


\section{References}

[1] S. Kasap, Optoelectronics and Photonics:Principles and Practices. Upper Saddle River, NJ: Prentice Hall, 2001.

[2] J. C. Campbell, B. C. Johnson, G. J. Qua, and W. T. Tsang, "Frequency response of inp/ingaasp/ingaas avalanche photodiodes," Journal of Lightwave Technology, , vol. 7, no. 5, pp. 778-784, 1989.

[3] J. Wu and G. Wang, "A novel equivalent circuit model for separate absorption grading charge multiplication avalanche photodiode (apd)-based optical receiver," Lightwave Technology, Journal of, vol. 28, no. 5, pp. 784$790,2010$.

[4] F. N. Najm, Circuit simulation. Hoboken, N.J: Wiley, 2010.

[5] HSPICE User Guide: Simulation and Analysis, Version a-2007.09 ed. USA: Copyright 2007 Synopsys, Inc., September 2007. 
[6] I. Synopsys, "Synopsys hspice," 2007.

[7] P. Gunupudi, T. Smy, J. Klein, and Z. J. Jakubczyk, "Self-consistent simulation of opto-electronic circuits using a modified nodal analysis formulation," Advanced Packaging, IEEE Transactions on, vol. 33, no. 4, pp. 979-993, 2010.

[8] H. W. Ruegg, "An optimized avalanche photodiode," Electron Devices, IEEE Transactions on, vol. 14, no. 5, pp. 239-251, 1967.

[9] E. W. Kamen and B. S. Heck, Fundamentals of signals and systems using MATLAB. Upper Saddle River, NJ: Prentice Hall, 1997.

[10] M. J. Roberts, Signals and systems : analysis using transform methods and MATLAB. Boston, MA ; Toronto: McGraw-Hill, 2004.

[11] S. An, M. J. Deen, A. S. Vetter, W. R. Clark, J. P. Noel, and F. R. Shepherd, "Effect of mesa overgrowth on low-frequency noise in planar separate absorption, grading, charge, and multiplication avalanche photodiodes," Quantum Electronics, IEEE Journal of, vol. 35, no. 8, pp. 1196-1202, 1999.

[12] M. Celik, L. Pileggi, and A. Odabasioglu, IC interconnect analysis. Boston, Mass: Kluwer Academic Publishers, 2002. 
[13] Y. Liu, L. T. Pileggi, and A. J. Strojwas, "ftd: an exact frequency to time domain conversion for reduced order RLC interconnect models," in Design Automation Conference, 1998. Proceedings, 1998, pp. 469-472.

[14] — - "Ftd: frequency to time domain conversion for reduced-order interconnect simulation," Circuits and Systems I: Fundamental Theory and Applications, IEEE Transactions on, vol. 48, no. 4, pp. 500-506, 2001.

[15] B. Gustavsen, "Improving the pole relocating properties of vector fitting," Power Delivery, IEEE Transactions on, vol. 21, no. 3, pp. 1587-1592, 2006.

[16] D. Deschrijver, M. Mrozowski, T. Dhaene, and D. D. Zutter, "Macromodeling of multiport systems using a fast implementation of the vector fitting method," Microwave and Wireless Components Letters, IEEE, vol. 18, no. 6 , pp. 383-385, 2008.

[17] B. Gustavsen and A. Semlyen, "Rational approximation of frequency domain responses by vector fitting," Power Delivery, IEEE Transactions on, vol. 14, no. 3, pp. 1052-1061, 1999.

[18] D. Saraswat, "Passive macromodeling of linear subnetworks characterized by measured/simulated data," M.A.Sc, Carleton University, Ottawa, ON, Canada, 2003. 
[19] E. Sackinger, Broadband circuits for optical fiber communication. Hoboken, NJ: Wiley-Interscience, 2005.

[20] S. Bandyopadhyay, S. E. Ralph, P. Mandal, and K. Pedrotti, "Integrated tia-equalizer for high speed optical link," in VLSI Design, 2008. VLSID 2008. 21st International Conference on, 2008, pp. 208-213. 